\title{
Les tortues du Pliocène d'Ahl al Oughlam (Casablanca, Maroc) et de localités mio-pliocènes avoisinantes
}

Saloua GMIRA
Rue 3, Immb 8, Appt 5, Hay Al Azhar, Av. Ibn Khatib, MA-30000 Fès (Maroc)
France DE LAPPARENT DE BROIN
Muséum national d'Histoire naturelle,
Département Histoire de la Terre,
CR2P, UMR 7207 CNRS-MNHN-UPMC,
case postale 38, 57 rue Cuvier, F-75231 Paris cedex 05 (France)
fdelap@mnhn.fr
Denis GERAADS

Muséum national d'Histoire naturelle, Département Histoire de la Terre,

CR2P, UMR 7207 CNRS-MNHN-UPMC,

case postale 38, 57 rue Cuvier, F-75231 Paris cedex 05 (France)

et Department of Human Evolution, Max Planck Institute for Evolutionary

Anthropology, Deutscher Platz 6, D-04103 Leipzig (Allemagne)

David LEFÈVRE

Université Paul Valery Montpellier III,

UMR 5140, Archéologie des Sociétés méditerranéennes,

390 avenue de Pérols, F-34970 Lattes (France)

Abderrahim MOHIB

Conservateur principal des Monuments et Sites,

Direction régionale de la Culture à Kénitra, MA-14000 (Maroc)

Jean-Paul RAYNAL

Université Bordeaux 1 Sciences et technologies, UMR 5199 PACEA,

IPGQ, Bât. B18, av. des Facultés, F-33405 Talence (France)

et Department of Human Evolution,

Max Planck Institute for Evolutionary Anthropology,

D-04103 Leipzig (Allemagne)

Gmira S., de Lapparent de Broin F., Geraads D., Lefèvre D., Mohib A. \& Raynal J.-P. 2013. Les tortues du Pliocène d'Ahl al Oughlam (Casablanca, Maroc) et de localités mio-pliocènes avoisinantes. Geodiversitas 35 (3): 691-733. http://dx.doi.org/10.5252/g2013n3a9

\section{RÉSUMÉ}

Le gisement pliocène supérieur d'Ahl al Oughlam (Casablanca, Maroc) a livré une faune de tortues cryptodires Testudinoidea, toutes affines de formes 


\section{MOTS CLÉS \\ Pliocène, Miocène, \\ Centrochelys, Testudo, \\ Mauremys, \\ Testudinoidea, Chelonii, espèces nouvelles.}

KEY WORDS

Pliocene,

Miocene,

Centrochelys,

Testudo,

Mauremys,

Testudinoidea,

Chelonii, new species. actuelles représentées en Afrique. Deux espèces terrestres et une amphibie sont reconnues. La première est une nouvelle petite espèce du genre paléarctique Testudo Linnaeus, 1758, T. oughlamensis $\mathrm{n}$. sp., apparentée à T. kenitrensis Gmira, 1993, du Pléistocène moyen de Kenitra (Maroc) avec laquelle elle constitue un nouveau clade au sein du genre. Elle est comparée à de nouveaux restes de Testudo de localités voisines et d'âge proche dont Dehar Mouak et Ar Rehamna. La seconde, ?Centrochelys marocana n. sp., est probablement apparentée à la forme actuelle C. sulcata (Miller, 1779), mais c'est une espèce géante. Le plus ancien représentant du genre au Maroc, provenant du gisement miocène supérieur/pliocène inférieur voisin de Lissasfa, est aussi examiné. Des comparaisons sont faites avec les grandes formes européennes et nord-africaines du Miocène à l'actuel. L'espèce d'eau douce, proche de l'espèce actuelle Mauremys leprosa (Schweigger, 1812), constitue le plus ancien représentant de ce genre, également paléarctique, au Maroc. La faune de tortues d'Ahl al Oughlam et de ses environs montre l'ancienneté au Maroc des trois genres actuels impliqués. Leur origine géographique est considérée.

\begin{abstract}
Turtles from the Pliocene of Ahl al Oughlam (Casablanca, Morocco) and nearby Mio-Pliocene localities.

The Late Pliocene locality Ahl al Oughlam (Casablanca, Morocco) yielded a fauna of cryptodiran testudinoid turtles, all of them related to extant African forms. Two terrestrial and one freshwater species are recognized. First, a new small species of the palaearctic genus Testudo Linnaeus, 1758, T. oughlamensis n. sp., is abundantly represented and is closely related to T. kenitrensis Gmira, 1993, from the Middle Pleistocene of Kenitra, Morocco. It is compared to other new remains of Testudo from nearby localities of similar age, including Dehar Mouak and Ar Rehamna. Together with T. kenitrensis, it constitutes a new clade within Testudo, providing a minimum age for the divergence between this clade and T. graeca Linnaeus, 1758 from Morocco. Secondly, the giant ?Centrochelys marocana n. sp., is probably related to the extant $C$. sulcata (Miller, 1779). Its description gives the opportunity to examine the earliest member of this genus in Morocco, from the nearby Late Miocene/Early Pliocene locality of Lissasfa. Comparisons with Miocene to extant genera of large size from Europe and northern Africa are made. The freshwater species from Ahl al Oughlam is close to the extant species Mauremys leprosa (Schweigger, 1812). It is the earliest record in Morocco of this second palaearctic genus. Thus, the fossil turtle fauna of Casablanca demonstrates the antiquity in Morocco of the three extant genera. Their geographical origin is considered.
\end{abstract}

\section{INTRODUCTION}

Le travail présenté ici s'inscrit dans le cadre de la description des faunes de vertébrés du gisement d'Ahl Al Oughlam: les tortues décrites ici représentent un nouvel article consacré à ce site.
Dans les formations pliocènes de la carrière que Biberson (1961) fit connaître sous le nom de "Carrière Deprez», le gisement d'Ahl al Oughlam, découvert en 1985, est exploité depuis 1989 (Raynal et al. 1990). Une abondante faune de vertébrés a été récoltée, incluant surtout des mammiferres (Geraads 


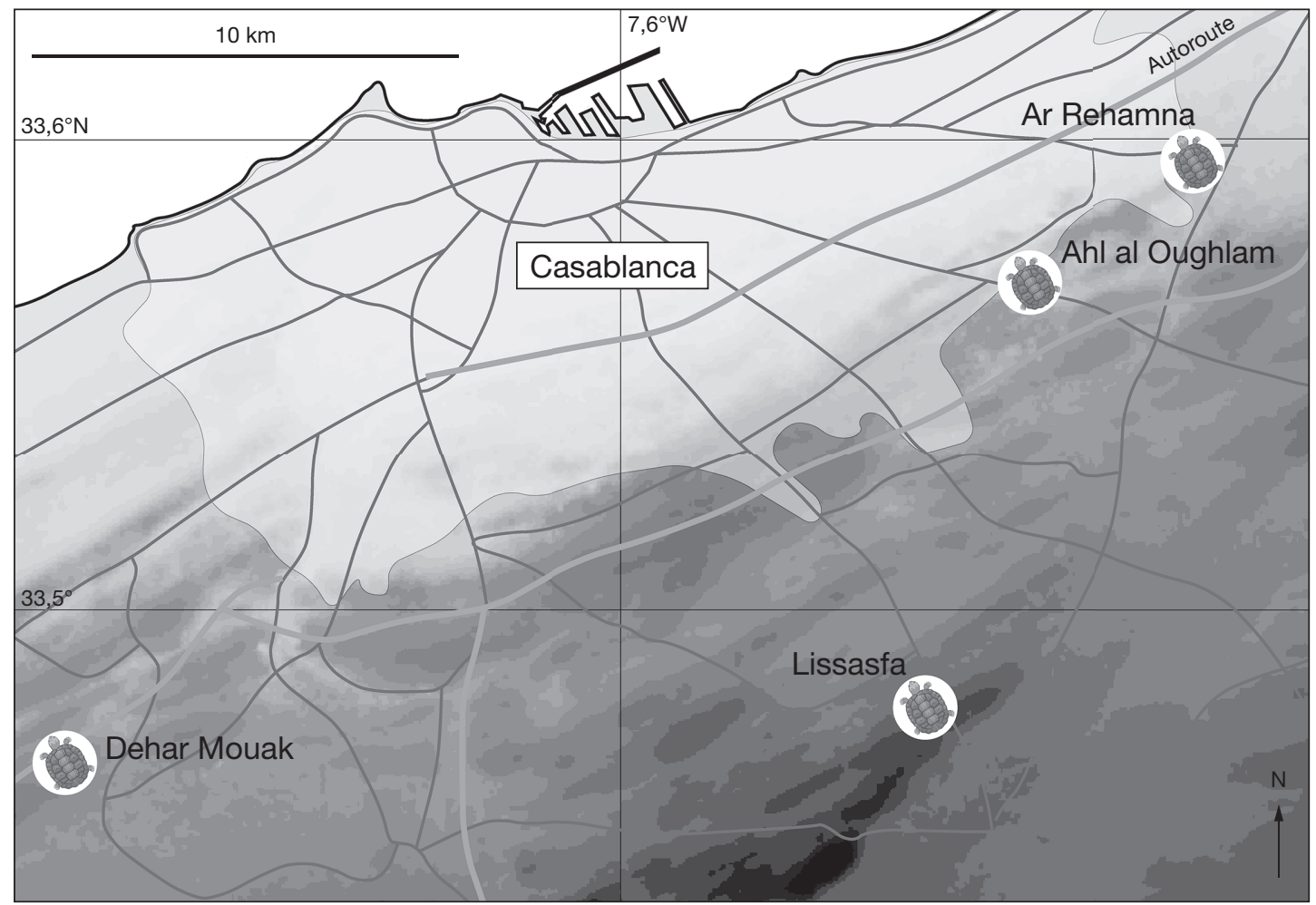

FIG. 1. - Carte de la région de Casablanca montrant la position des localités ayant livré des tortues étudiées. Dehar Mouak, Ahl al Oughlam et Ar Rehamna appartiennent au même système dunaire pliocène, tandis que Lissasfa est topographiquement plus haut mais plus ancien (limite Miocène supérieur/Pliocène inférieur).

1989, 2006, 2011; Eisenmann \& Geraads 2007; Geraads et al. 2010; Gunnell et al. 2011), mais aussi des amphibiens, des squamates et un crocodile (Bailón 2000) et de nombreux oiseaux (MourerChauviré \& Geraads 2008, 2010 ; Geraads 2010c). La présence de deux tortues terrestres, dont une grande, a été mentionnée par Raynal et al. (1990), Bailón (2000) et Lapparent de Broin (2000a), mais sans description ni preuves de leur détermination. Elles sont étudiées ici, ainsi que deux restes d'une troisième tortue, d'eau douce, découverte plus récemment. Cette faune de tortues d'Ahl al Oughlam comporte des représentants de deux sousfamilles de Testudinoidea Batsch, 1788. Leur étude donne l'opportunité de décrire d'autres restes de la superfamille provenant de localités voisines dont $\mathrm{Ar}$ Rehamna et Dehar Mouak (Pliocène terminal) et Lissasfa (proche de la limite Mio-Pliocène) : elles ont été plus récemment découvertes dans la même région des cordons dunaires de Casablanca. Le présent travail constitue un complément à l'étude des tortues inclues dans la synthèse des tortues fossiles du Maroc de Gmira (1995). Il permet de faire le point sur leur colonisation du Maroc au Néogène.

\section{MATÉRIEL ET MÉTHODES}

\section{CADRE GÉOLOGIQUE ET}

MORPHO-STRATIGRAPHIQUE

La région de Casablanca est caractérisée par un modelé de grands cordons. À chacun de ces cordons sont associés des dépôts intertidaux à supratidaux (rudites et biocalcarénites) et dunaires (biocalcarénites) caractéristiques de séquences littorales régressives corrélatives des variations de l'océan, 
et des faciès d'altération (karsts, paléosols) et de remaniement. Pour les cordons les plus anciens, les carrières et les affleurements sont éloignés les uns des autres et bien souvent il n'y a qu'un lieu d'observation par cordon ce qui rend les corrélations stratigraphiques difficiles à établir. Cependant, la cartographie du contact calcarénites/substrat a permis de mettre en évidence plusieurs unités morpho-sédimentaires (UMS), souvent complexes, formées de un ou plusieurs cordons coalescents et associées à de grandes plateformes étagées développées aux dépens du substrat quartzitique (Lefèvre 2000 ; Lefèvre \& Raynal 2002).

Dans la partie la plus ancienne de ce système, dans l'état actuel des données, on distingue quatre unités morpho-sédimentaires:

- l'UMS de Médiouna/Bouskoura, la plus ancienne. Elle correspond au grand cordon de Marchich qui culmine à $244 \mathrm{~m}$ et domine la plaine de Médiouna. Il s'agit d'une unité complexe qui regroupe plusieurs cordons vraisemblablement associés à plusieurs plateformes étagées entre $190 \mathrm{~m}$ et $150 \mathrm{~m}$ d'altitude. Dans le remplissage d'une poche karstique développée au sommet d'une des formations les plus récentes de l'UMS de Médiouna/Bouskoura, a été découvert en 1995 le gisement paléontologique de Lissasfa (Raynal et al. 1999), dont les éléments fauniques comportent quelques restes de grands mammifères avec une riche microfaune (Geraads 1998), et un reste de tortue décrit ici. Les caractères de l'association de micromammifères la situent vers la limite Mio-Pliocène, aux environs de 5,5 Ma, et fixent donc un âge minimum Miocène final pour la formation de Médiouna;

- l'UMS de Dehar Mouak est associée à une plateforme située entre 130/135 m d'altitude, emboitée dans l'ensemble précédent. Sa partie supérieure est formée par une biocalcarénite à litage planaire fortement incliné $\left(34^{\circ} \mathrm{N} 100\right)$, altérée en surface, qui constitue l'essentiel du cordon. C'est de la partie supérieure altérée de cette éolianite que provient une carapace de tortue décrite ici; - l'UMS d'Oulad Malik est un complexe de cordons associés à une plateforme située à 115/110 m d'altitude, emboîté dans l'ensemble précédent;
- l'UMS de Sidi Messaoud est un complexe de cordons associés à une ou plusieurs plateformes rencontrées entre 90 et $100 \mathrm{~m}$ d'altitude. Appartiennent à ce complexe les formations rencontrées à Ahl al Oughlam, ainsi que dans la carrière d'Ar Rehamna, entaillée dans le cordon d'Al Hrawiyine, localités qui ont livré des tortues décrites ici. Les données biostratigraphiques déduites de l'étude des faunes du gisement d'Ahl al Oughlam, associé à un paléorivage de cette formation, le situent autour de 2,5 Ma (Raynal et al. 1990 ; Geraads 2006, 2010a, b), mais le site d'Ar Rehamna, par sa position dans le dispositif morphologique, pourrait être un peu plus récent.

Les UMS de Oulad Malik et Dehar Mouak appartiennent donc au Pliocène, Dehar Mouak pouvant être du Pliocène inférieur. Les formations de l'UMS de Sidi Messaoud peuvent être rattachées au Pliocène supérieur.

\section{Les sites mio-pliocènes des cordons dunaires}

Carrière d'Ahl al Oughlam. Situé en bordure sud-est de la ville de Casablanca (Fig. 1), le site est daté d'environ 2,5 millions d'années, vers le sommet du Pliocène. Il est constitué par le remplissage d'un ensemble de fissures de même âge, d'après l'étude des micromammifères, entre des blocs d'éolianites (Geraads 1995).

La faune de tortues est presque exclusivement constituée par les formes terrestres et principalement par une petite espèce de Testudo Linnaeus, 1758, genre bien représenté au Maroc actuellement. Le premier travail sur les tortues du Maroc (Gmira 1995) ne comportait pas de description de matériel de Testudinidae Batsch, 1788 antérieur au Pléistocène moyen. À Ahl al Oughlam se trouve une petite espèce de Testudo très proche de T. kenitrensis Gmira, 1993a, du Pléistocène moyen de Kenitra (Tableau 1). Elle est bien représentée, par un nombre suffisant de spécimens pour pouvoir à la fois distinguer les deux espèces entre elles et par rapport à $T$. graeca Linnaeus, 1758, présente actuellement au Maroc, et pour pouvoir associer ces deux formes fossiles marocaines dans un groupe kenitrensis où elles constituent un clade bien différencié dans le genre Testudo. A côté de la petite Testudo, se trouvent à Ahl al Oughlam 
quelques éléments d'une tortue géante pouvant atteindre 180 à $200 \mathrm{~cm}$ de longueur de carapace, et appartenant probablement au genre Centrochelys Gray, 1872; celui-ci a pour espèce type C. sulcata (Miller, 1779), la plus grande tortue terrestre d'Afrique, actuellement bien plus petite toutefois que celle fossile du Maroc puisque ne pouvant atteindre qu'environ $90 \mathrm{~cm}$ de longueur de carapace. Enfin deux restes de carapace de tortues d'Ahl al Oughlam sont attribués au genre actuel amphibie Mauremys Gray, 1869, toujours présent actuellement dans ce pays avec $M$. leprosa (Schweigger, 1812) (Schleich 1996; Bour \& Maran 1998). Ce genre était déjà connu fossile au Maroc mais pas avant le Pléistocène supérieur de Taforalt et de Doukkala 2 (Gmira 1995; Lapparent de Broin 2000a) (Tableau 1).

Autres localités côtières pliocènes du même système de cordons dunaires. Ar Rehamna de l'UMS de Sidi Messaoud comme Ahl Al Oughlam, Dehar Mouak de l'UMS éponyme, et le sommet d'un cordon dunaire des mêmes environs de Casablanca non daté ni localisé précisément, ont livré des restes inédits de Testudo pliocènes.

Lissasfa, site miocène terminal de l'UMS de Médiouna/Bouskoura. Un spécimen inédit de Centrochelys sp. atteste la présence du genre au Maroc dès la fin du Miocène vers 5,5 Ma, avant $\mathrm{Ahl}$ al Oughlam.

\section{Remarque}

Toutes ces tortues sont étudiées par comparaison avec les représentants fossiles et actuels (MNHN.F, ZA, RA) des genres concernés, dans leur contexte géographique et géologique. Au sein des Testudinidae, les Testudininae sont restreints ici aux formes terrestres, les «Testudininei » de nos travaux précédents, et n'incluent pas ici les Geoemydinei Theobald, 1868, les deux subdivisions étant élevées ici au rang de sous-familles, lesquelles sont paraphylétiques. Les Testudininae sont en effet issus des Geoemydinae comme cela a été démontré sur l'anatomie squelettique de la famille par analyse cladistique (Hirayama 1985) et de celle des représentants les plus primitifs (Lapparent de Broin et al. 2006a, b).
TABleAU 1. - Chronologie approximative des principaux sites mentionnés dans le texte. Ceux situés en Europe sont en italiques.

\begin{tabular}{ll}
\hline Pléistocène & Taforalt \\
& Doukkala \\
& Oualidia \\
& Kenitra \\
& Tighenif \\
& Mansourah \\
& Aïn Boucherit \\
\hline Pliocène & Ahl al Oughlam \\
& Ichkeul \\
& Perpignan \\
& Wadi Natrun \\
& Hamada Damous \\
\hline Miocène & Lissasfa \\
supérieur & Lubéron \\
& Baynunah Fm \\
& Pikermi \\
& Samos \\
& Djebel Krechem \\
& Ravin de la Pluie \\
& Djebel Sémène \\
& Bou Hanifia \\
\hline Miocène & Artenay \\
inférieur & Djebel Zelten \\
& As Sarrar \\
\hline &
\end{tabular}

ABRÉVIATIONS

INSAP Institut national des Sciences de l'Archéologie et du Patrimoine, Rabat;

MHNT Muséum d'Histoire naturelle de Toulouse; MNHN Muséum national d'Histoire naturelle, Paris; MNHN.F Collections de Paléontologie;

MNHN.ZA Collections d'Anatomie comparée;

MNHN.RA Collections de Zoologie des Reptiles et Amphibiens.

\section{SYSTÉMATIQUE}

Ordre CHELONII Latreille, 1800

Super-famille TESTUDINOIDEA Batsch, 1788

Famille Testudinidae Batsch, 1788

Sous-famille TeSTUdininaE Batsch, 1788

Genre Testudo Linnaeus, 1758

Groupe T. kenitrensis

Testudo oughlamensis $\mathrm{n} . \mathrm{sp}$.

(Figs 2-6)

Testudo - Bailón 2000: 542. 
Testudo aff. kenitrensis - Lapparent de Broin 2000a: 62.

HOLOTYPE. - Collection INSAP. AaO-272, carapace incomplète: bord antérieur légèrement érodé aux périphériques 1 et 2 droites et à la nucale. Erosion des neurales 1 et 2 , des parties arrière de la pleurale 2 et antérieure de la pleurale 3 droites, de la partie latérale des pleurales 2 et 3 gauches, et de l'arrière: majeure partie des pleurales 8 , les suprapygales, la pygale et une grande partie de la bordure périphérique arrière (de la partie postérieure de la périphérique 7 gauche jusqu'à la partie postérieure de la périphérique 6 droite) et périphériques 2 à 5 gauches. Plastron sans les xiphiplastrons. Le moule interne est conservé par places permettant de suivre les contours de plaques manquantes.

PARATYPES. - Collection INSAP. Au moins dix individus. AaO-948: carapace incomplète et fragmentée représentée par la moitié gauche de la nucale avec la pleurale 1 gauche, une partie de la pleurale 1 droite, les neurales 3 quadrangulaire et 4 octogonale avec la partie proximale des pleurales 3 et 4 gauches et 3, 4 et 5 (partielle) droites, les neurales 7 et 8 ; attachées au plastron, les périphériques 3,4 et 6 à 8 gauches et 3 et 6 à 8 droites avec la partie distale de la pleurale 8 droite; des fragments de pleurales paires et impaires; le plastron incomplet médialement (morsure animale probable par comparaison avec des blessures identifiées comme telles sur des spécimens actuels; coll. MNHN.F, RA, ZA) et sans le xiphiplastron droit. AaO-949: une carapace incomplète assez jeune et fragmentée représentée par la nucale avec les périphériques 1 et 2 gauches et droites et une partie de la pleurale 1 gauche, sept périphériques, trois fragments de pleurales (paires et impaires), la pygale, le plastron dont manque la région latérale de l'hypoplastron gauche et celle médiale de l'hypoplastron gauche et avec les périphériques 6 à 8 gauches et 5 et 6 droites; deux fragments de diaphyses d'un humérus et d'un coracoïde. Fragments isolés trouvés avec AaO-948 et AaO-949: deux neurales paire ( $6 \mathrm{e}$ ?) et impaire (7e ?); une périphérique 11 avec pleurales 8 gauche et 6 et 7 droites; deux périphériques du pont et deux périphériques postérieures; deux portions proximales et trois portions médianes de pleurales paires $\left(2 \mathrm{e}, 4^{\mathrm{e}}\right.$ ou $\left.6^{\mathrm{e}}\right)$ et un fragment distal de pleurale paire; deux portions distales de pleurales paire et impaire associées et un fragment distal de pleurale paire; trois fragments de pleurales impaires; un fragment distal de pleurale 8 gauche avec un fragment de plastron. $\mathrm{AaO}-293$, périphérique du pont $\left(5^{\mathrm{e}} \mathrm{ou} 6 \mathrm{e}\right)$, périphérique postérieure (10e) et neurale 8; AaO-645, hypoplastron gauche; $\mathrm{AaO}-659$, périphérique du pont (8e gauche); $\mathrm{AaO}-672$, périphérique du pont, $8 \mathrm{e}$ gauche; pleurale 8 gauche (partie distale), épiplastron gauche et hypoplastron droit; AaO-673, -674, -675, trois épiplastrons avec leur entoplastron; AaO-676 et -677, deux fragments d'entoplastrons; $\mathrm{AaO}-678$, cinq fragments de périphériques; AaO-679, fragment d'hypoplastron; AaO-680, fragment d'hypoplastron gauche; $\mathrm{AaO}-681, \mathrm{AaO}-682$, fragments d'hypoplastron gauche; AaO-683, hypoplastron gauche; $\mathrm{AaO}-684$, fragment d'hypoplastron droit; $\mathrm{AaO}-685$, fragment d'hypoplastron gauche; $\mathrm{AaO}-686$, fragment latéral d'hyoplastron droit; $\mathrm{AaO}-687$, fragment de nucale; $\mathrm{AaO}-688$, xiphiplastron gauche; $\mathrm{AaO}-689$, pygale; $\mathrm{AaO}-$ 690, périphérique 11 gauche; $\mathrm{AaO}-691$, périphérique 1 gauche; $\mathrm{AaO}-692$, périphérique 11 gauche; $\mathrm{AaO}-693$, entoplastron; $\mathrm{AaO}-694$, pygale; $\mathrm{AaO}-695$, périphérique 2 gauche; AaO-696, humérus droit partiel; AaO-697, périphérique 11 gauche avec pygale; $\mathrm{AaO}-698$, pygale; AaO-699, pygale; AaO-2113, périphérique 1 gauche; $\mathrm{AaO}-2114$, périphérique 9 gauche; $\mathrm{AaO}-2115$, neurale 5; $\mathrm{AaO}-2116$, périphérique 2 gauche; $\mathrm{AaO}-2117$, fragment de pleurale 1 gauche; $\mathrm{AaO}-2118$, fragment de pleurale 1 droite; AaO-2119 fragment de pleurale; AaO-2120 pleurale $8 ; \mathrm{AaO}-2121, \mathrm{AaO}-2122$, périphériques du pont (5e-6e) gauche; $\mathrm{AaO}-2123,16$ lots de plaques comportant en tout 34 périphériques, 31 pleurales, 7 neurales et 17 fragments non situés; $\mathrm{AaO}-2124$, trois périphériques antérieure, du pont et postérieure, jeunes; AaO-2125, trois périphériques postérieures jeunes; $\mathrm{AaO}-2127$, épiplastron gauche; $\mathrm{AaO}-2129$, périphérique 9 gauche; $\mathrm{AaO}-2130$, périphérique 10 gauche; $\mathrm{AaO}-4788$, nucale; $\mathrm{AaO}-4789$, hyoplastron gauche; $\mathrm{AaO}-4790$, hyoplastron droit. Des fragments associés dans un lot peuvent appartenir à un même individu.

ÉTYMOLOGIE. - En référence au gisement $\mathrm{Ahl}$ al Oughlam.

LOCALITÉ ET HORIZON STRATIGRAPHIQUE TYPES. Remplissage continental dans la Carrière d'Ahl al Oughlam, située au sud-est de Casablanca, à gauche de la route S 106 allant de Casablanca à Tit Mellil. $33^{\circ} 34^{\prime} 11^{\prime \prime} \mathrm{N}, 07^{\circ} 30^{\prime} 44^{\prime \prime} \mathrm{W}$. Cordons dunaires de la côte de Casablanca à Kenitra, Maroc. Unité morpho-sédimentaire de Sidi Messaoud. Carte géologique, feuille de Casablanca-Mohammedia (Destombes 1987). Pliocène supérieur, âge estimé à 2,5 Ma (Raynal et al. 1990 ; Geraads et al. 2010).

FIG. 2. - Testudo oughlamensis n. sp., Ahl al Oughlam, Maroc, Pliocène supérieur, coll. INSAP : A, AaO-272, holotype, carapace : A1-A4 : faces dorsale, dorso-antérieure, latérale droite, ventrale; A5, A6, extrémité du lobe antérieur, faces dorsale et latérale droite ; B, AaO-695 ; B1, B2, périphérique 2 gauche, faces dorsale et ventrale ; C, AaO-691; C1, C2, périphérique 1 gauche, faces dorsale et ventrale ; $\mathbf{D}, \mathrm{AaO}-4788$, nucale, faces dorsale et ventrale ; E, AaO-697, périphérique 11 gauche et pygale mâle, faces dorsale et ventrale; F, AaO-692 ; F1, F2, périphérique 11 droite, faces ventrale et dorsale ; G, AaO-698, pygale femelle, faces ventrale et dorsale ; H, AaO-2128; pygale femelle, faces dorsale, latérale gauche et ventrale ; I, AaO-694, pygale mâle, faces dorsale et latérale gau- 


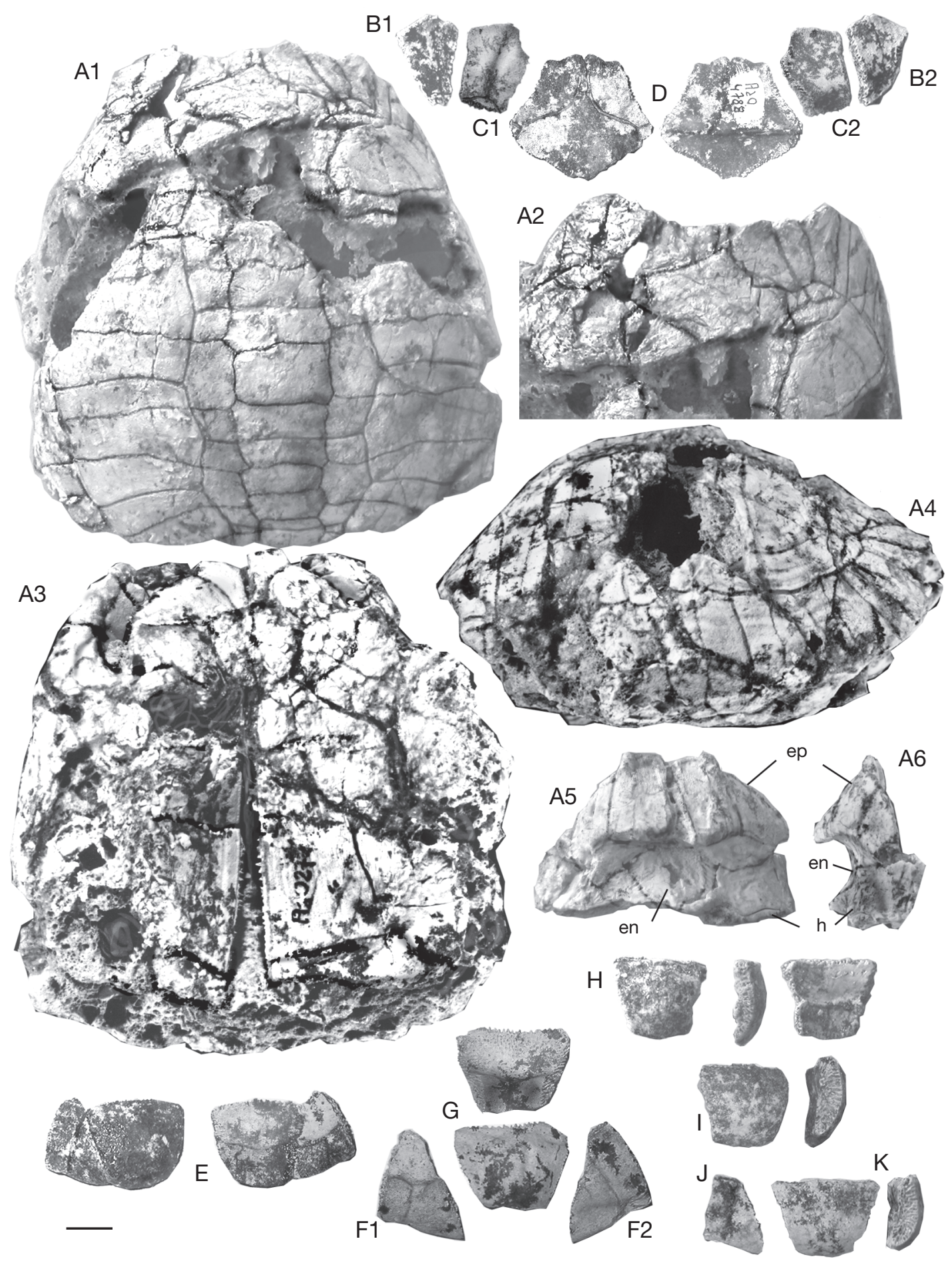

che ; J, AaO-690, périphérique 11 gauche, face dorsale ; K, AaO-699, pygale mâle, faces dorsale et latérale gauche. Abréviations : en, entoplastron ; ep, bourrelet épiplastral ; $\mathbf{h}$, hyoplastron. La flèche indique l'encoche nucale à la périphérique 1 . Échelle : $1 \mathrm{~cm}$. 
DiAGNOSE. - Espèce de Testudo formant notamment avec T. kenitrensis un groupe primitif par la bordure périphérique non redressée et la participation préservée de l'hypoplastron au lobe postérieur, à sillon abdominofémoral sinueux plus ou moins séparé de la charnière hypo-xiphiplastrale médialement et la rejoignant ou non latéralement et seulement sur le bord dorsal; dérivé par la petite taille de la carapace (ici $13 \mathrm{~cm}$ maximum) et le contour quadrangulaire dilaté médialement en vue dorsale, très arrondi en profil latéral: fortement convexe d'avant en arrière entre, d'une part, l'avant sous la nucale et les écailles vertébrales 1 et 2 , et, d'autre part, l'arrière plus abruptement incliné, sous les écailles vertébrales 3 à 5, sans long plateau intermédiaire; par l'encoche nucale profonde, trapézoïdale, entre les périphériques 1 à la jonction des marginales 1 et 2 ; et par les vertébrales étroites. Formant avec T. kenitrensis un clade par le bourrelet épiplastral dorsal légèrement concave, épais, long et soulevé en corniche à l'arrière au dessus d'une petite poche gulaire et les anales longues, rapprochées médialement de la suture hypo-xiphiplastrale. Se distinguant de T. kenitrensis par le contour plus dilaté médialement en vue dorsale et les écailles vertébrales 2,3 et 4 relativement plus étroites; le bord antérieur de la nucale en saillie vers l'avant au milieu de l'encoche; la vertébrale 1 recouvrant ou non les coins de la nucale, les bords latéraux de cette vertébrale 1 variant de rectilignes (divergents vers l'avant) à légèrement arrondis et à sinueux (vertébrale en lyre), alors que la vertébrale en lyre est constante chez T. kenitrensis; le plastron à lobe antérieur plus allongé aux épiplastrons et le bourrelet épiplastral dorsal un peu moins long; la relativement plus étroite petite saillie gulaire par rapport aux humérales; le sillon fémoro-anal plus rapproché médialement de la suture hypo-xiphiplastrale et la présence de deux écailles inguinales au lieu d'une.

\section{DESCRIPTION}

\section{Holotype}

Longueur préservée: $106 \mathrm{~mm}$ (estimée à $120 \mathrm{~mm}$ maximum); largeur: $101 \mathrm{~mm}$; hauteur: $65 \mathrm{~mm}$. Vertébrale 3, largeur : $4 \mathrm{~mm}$ sur longueur : $28 \mathrm{~mm}$. Bien qu'incomplet, c'est le seul spécimen montrant la forme globale de la carapace, apomorphe (Figs 2; 3). Sa partie postérieure manquant et sa partie antérieure étant légèrement érodée, sa description est complétée par les paratypes.

Carapace dorsale. Le spécimen est un peu déformé et déjeté sur la droite, la bordure latérale apparaissant de ce fait un peu à gauche vers l'arrière (Figs 2; 3), et le plastron est un peu enfoncé sous la dossière mais ses éléments sont plats, pouvant indiquer qu'il s'agit d'une femelle. L'épaisseur des plaques et des annuli de croissance montre qu'il s'agit d'un spécimen bien adulte. Le profil de la carapace en vue latérale (Fig. 2A4) montre une forme courte avec un contour arrondi, présentant une pente forte vers l'avant et une un peu plus forte à l'arrière, avec un court plateau intermédiaire à peu près horizontal sous la vertébrale 3 , avec juste deux petites bosselures aux neurales 2 et 4 (sous les vertébrales 2 et 3 ) et de petites bosses aussi sous la vertébrale 4 et médialement sous les costales 2 et 3 . La face dorsale montre également un contour arrondi, de chaque côté. En vue dorsale, la bordure périphérique antérieure (la postérieure non conservée) suit la pente du disque pleural sans se relever vers son extrémité. La nucale est un peu incomplète à gauche et séparée postmortem de la périphérique 1 gauche; elle est plus large que longue, hexagonale resserrée à l'arrière au contact avec la neurale 1 (la plus étroite de la série neurale). Le bord antérieur de la dossière forme une encoche entre les périphériques 1 (aux sillons entre marginales 1 et 2) avec la nucale en retrait (Fig. 2A2), encoche amoindrie ici par l'érosion du bord des plaques, mais complète sur le spécimen AaO-249 (Fig. 4B) et estimable d'après la nucale et les périphériques 1 et 2 isolées (Fig. 2B-D). Les périphériques antérieures 1 et 2 sont longues, étroites dans leur extrémité postérieure (médiale) et s'élargissent vers l'avant. Leur bord libre externe, complété par les paratypes (Figs 2; 4), était saillant en pointe à la jonction des écailles marginales 1-2 et 2-3 respectivement. Les périphériques antérieures sont en pente oblique suivant la pente du disque pleural, sans se redresser vers leur extrémité; les périphériques du pont étaient verticales : comme le spécimen a été déformé post-mortem et fléchi sur la gauche, les périphériques du pont apparaissent artificiellement en partie à gauche. Les neurales 2 à 5 sont différenciées mais très irrégulières: neurale 1 (manquante mais estimée par sa place entre les plaques environnantes) quadrangulaire; neurale 2 octogonale (son bord droit manquant); 3 e heptagonale (le petit côté latéro-antérieur gauche manquant); 4 e pentagonale avec un petit côté latéro-postérieur droit ; $5 \mathrm{e}$ hexagonale avec un petit côté latéro-antérieur gauche et un petit côté latéro-postérieur droit; $\sigma^{\mathrm{e}}$ pentagonale avec un petit côté latéro-antérieur gauche; 7e hexagonale avec petits côtés latéro-anté- 

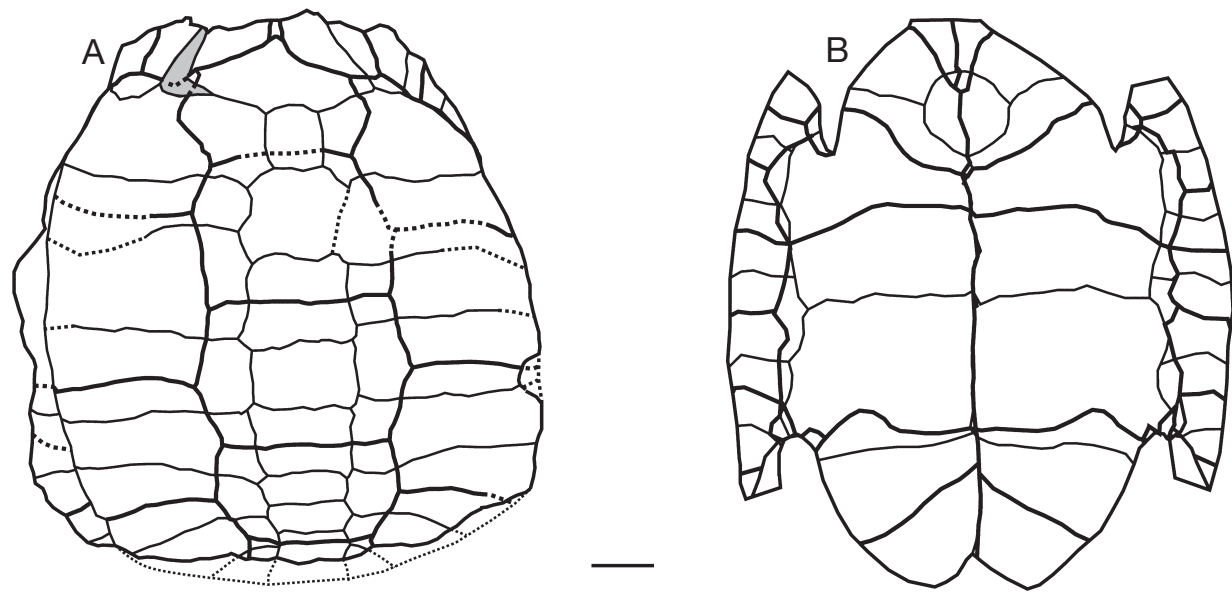

FIG. 3. - Testudo oughlamensis n. sp., Ahl al Oughlam, Maroc, Pliocène supérieur, coll. INSAP : A, AaO-272, holotype, schéma de la carapace (sans la bordure postérieure), face dorsale; $\mathrm{B}, \mathrm{AaO}-448$, reconstitution du plastron avec le pont (périphériques 3 à 8). Échelle : $1 \mathrm{~cm}$.

rieurs; 8e hexagonale avec petit côté latéro-postérieur droit mais petit côté antérieur gauche aligné sur le petit côté postérieur gauche (séparés par la jonction pleurales 7 et 8). La différentiation irrégulière des neurales entraîne une irrégularité des pleurales dans leur longueur respective. Les pleurales 2 à 6 sont alternativement plus courtes et plus longues (d'avant en arrière) médialement et plus longues et plus courtes latéralement, mais irrégulièrement à cause de l'irrégularité des neurales, et les pleurales 2 droite et 3 gauche sont particulièrement longues. Les périphériques 3 à 7 du pont, partiellement érodées (surtout à gauche), sont hautes, leurs parties dorsale et ventrale étant très ouvertes en un angle de plus de $90^{\circ}$ (Fig. 2A4); leur base ventrale est un peu enfoncée avec le plastron.

Écailles: la cervicale est assez longue sur la nucale (23\%); très étroite dorsalement, elle s'étrécit un peu à l'avant et son bord arrière est concave; elle est un peu plus large ventralement. La 1 re marginale est un peu plus courte que la périphérique 1 ; les marginales suivantes sont aussi longues que les périphériques, la suture périphériques-pleurales coïncidant avec le sillon marginales-costales. Les quatre écailles préservées de la série vertébrale sont plus larges que longues, hexagonales, mais la série est relativement modérément large; la largeur sur longueur de la vertébrale 3 (la plus large) est de
$142 \%$ (152-155\% chez T. graeca). La 1 re est partiellement endommagée médialement; elle est en forme de lyre (bords latéraux sinueux) : à partir de l'arrière, ses bords latéraux divergent vers l'avant, s'arrondissent en se rapprochant (étrécissement de l'écaille) pour continuer en s'écartant à peine et arriver sur la nucale en ne recouvrant pas les coins postéro-latéraux. Le rebord de la partie visible ventralement des marginales antérieures et de la cervicale est long ( $14 \mathrm{~mm}$ à la cervicale). Une écaille axillaire est visible à droite dans sa partie située sur la périphérique 3 (complète sur le spécimen AaO-948, Fig. 4A2). Les annuli de croissance des écailles sont particulièrement visibles sur les pourtours pleural et périphérique droits.

Plastron. Longueur préservée (de l'avant des épiplastrons à l'arrière des hypoplastrons) : $93 \mathrm{~mm}$; longueur et largeur du lobe antérieur : 56 et $37 \mathrm{~mm}$; largeur et longueur de la saillie gulaire: $22 \mathrm{~mm}$ et $5 \mathrm{~mm}$; longueur et hauteur du bourrelet épiplastral dorsal: $22 \mathrm{~mm}$ et $16 \mathrm{~mm}$; largeur des deux gulaires à l'arrière du bourrelet épiplastral dorsal: $28 \mathrm{~mm}$. Longueur du pont: $64 \mathrm{~mm}$. Le lobe antérieur est trapézoïdal avec une rupture de pente: ses bords latéraux sont d'abord un peu convergents (angle à l'encoche axillaire assez fermé, $c .35^{\circ}$ ) puis ses bords convergent davantage pour se resserrer encore à la 
saillie gulaire, nette. Il est nettement plus étroit que le lobe postérieur. Le lobe antérieur est relativement long du fait des épiplastrons allongés: le rapport de la longueur du bord de l'épiplastron à la longueur du bord latéral du lobe antérieur est de $69 \%$ (T. graeca: $48 \%$ ). Dorsalement, les épiplastrons s'élèvent et se courbent vers l'arrière un peu en corniche jusqu'au-dessus de l'avant de l'entoplastron: ce bourrelet est élevé et s'incurve un peu, se faisant légèrement concave. L'entoplastron a un aspect rhombique, côtés latéro-postérieurs arrondis, aussi long que large; il est petit dans le lobe antérieur et atteint ou dépasse à peine postérieurement le fond des encoches axillaires. Le pont est long et massif. Les hypoplastrons participent un peu au lobe postérieur. Les xiphiplastrons manquent mais le bord postérieur libre des hypoplastrons a une surface denticulée qui montre l'existence d'une charnière hypo-xiphiplastrale.

Écailles: les gulaires sont saillantes latéralement par rapport aux humérales mais elles ne sont pas en relief ventralement par rapport à ces dernières; ventralement triangulaires, elles s'étrécissent un peu en coin, en s'incurvant, atteignant la pointe de l'entoplastron. Dorsalement, elles couvrent le bourrelet épiplastral dorsal en s'élargissant un peu vers l'arrière. Le sillon huméro-pectoral contourne l'entoplastron, éloigné latéralement et plus près médialement tout en restant bien à l'écart de la plaque. Médialement derrière l'entoplastron, se font de plus en plus grandes, se multipliant par deux en longueur chaque fois: les humérales, les pectorales et les abdominales. Les abdominales ne parviennent pas à la suture hypoplastrale postérieure médialement et forment une sinuosité convexe de chaque côté. L'abdominale droite est incomplète latéralement mais la gauche est préservée: elle rejoint presque la suture-charnière latéralement en arrière d'une petite écaille inguinale hypoplastrale. Des annuli de croissance des écailles sont visibles sur l'hypoplastron.

\section{Paratypes}

Ils sont indispensables pour compléter l'holotype et pour appréhender la variabilité spécifique. Les caractères essentiels de l'holotype sont confirmés par les plaques isolées et les spécimens AaO-948 et $\mathrm{AaO}-949$ (Figs 2 ; 3B ; 4-5). Les plastrons de ces deux spécimens sont plats et devaient appartenir à des femelles, ce que confirme la pygale conservée du second, non épaissie et peu recourbée.

Carapace dorsale. La nucale complète est préservée par les spécimens $\mathrm{AaO}-4788$ (Fig. 2D), AaO-948 (Fig. 4A4) et le plus jeune AaO-949 (Fig. 4B1, $\mathrm{B} 2$ ), celui-ci montrant l'encoche nucale dans son intégralité. Les plaques neurales isolées et celles de AaO-948 montrent comme l'holotype la différenciation de neurales médianes, régulière dans la partie préservée chez AaO-948: 3e quadrangulaire, $4^{\mathrm{e}}$ octogonale et $5^{\mathrm{e}}$ quadrangulaire, alors que cette partie est irrégulièrement différenciée chez l'holotype. Plusieurs périphériques antérieures sont préservées dont la première d'AaO-691 (Fig. 2C) et d'AaO-949 (Fig. 4B) avec la seconde d'AaO-695 (Fig. 2B) et d'AaO-649 (Fig. 4B) : elles montrent aussi l'encoche profonde trapézoïdale du bord antérieur, avec les périphériques 1 saillantes à bord médial encoché (concave), et la nucale projetée vers l'avant au milieu; les plaques périphériques 1 et 2 et la nucale forment une pointe à la jonction externe des marginales entre elles et avec la cervicale. La périphérique 2 s'épaissit postérieurement au contact de la 3 e qui ouvre le pont. Le pont est bien visible chez $\mathrm{AaO}-948, \mathrm{AaO}-949$ et plusieurs plaques isolées: les plaques du pont sont hautes à angle obtus au milieu du pont. La périphérique 3 porte le contrefort axillaire de l'hyoplastron dressé verticalement et un peu en oblique contre sa moitié antérieure épaissie et le début du pont à sa moitié postérieure, s'ouvrant largement sur les périphériques 4 à 6 ouvertes en angle obtus. La périphérique 7 ferme le pont et porte dans sa moitié postérieure le contrefort inguinal hypoplastral dressé verticalement et débordant un peu sur le bord ventroantérieur de la périphérique 8 , verticale, dont la face externe est un peu bombée et dont le bord ventral est libre comme les suivantes. Les postérieures sont conservées en plusieurs exemplaires: la périphérique 9 (Fig. 5O1), également bombée extérieurement, se fait plus courte et s'épaissit postérieurement comme l'avant de la 10e (Fig. 5O2) laquelle s'amincit postérieurement à l'épaisseur de la $11^{\mathrm{e}}$ et de la pygale. Ces plaques postérieures sont 

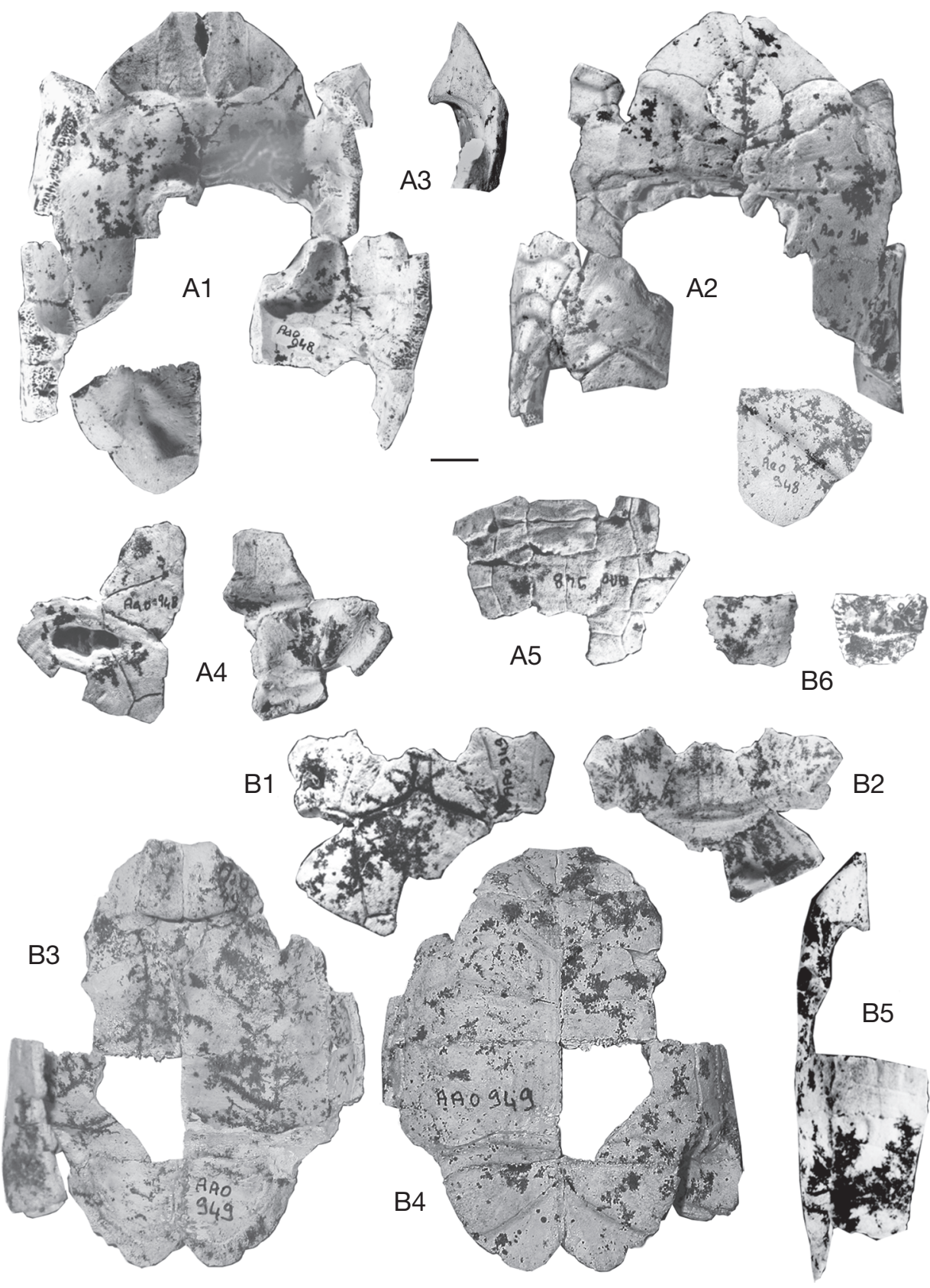

FIG. 4. - Testudo oughlamensis n. sp., Ahl al Oughlam, Maroc, Pliocène supérieur, coll. INSAP : A, AaO-948, A1-A6, quelques éléments de la dossière partielle avec plastron; A1, A2, plastron avec périphériques 3, 4 et 6 à 8 gauches, et 3 et 6 à 8 droites avec fragment de pleurale 8, faces dorsale et ventrale ; A3 lobe antérieur en vue latérale gauche; A4, demi-nucale avec pleurale 1 gauche, faces dorsale et ventrale; $\mathbf{A 5}$, neurales 3 et 4 avec pleurales adjacentes partielles, face dorsale ; $\mathbf{B}, \mathbf{A a O}-949$; B1-B6, dossière partielle avec plastron ; B1, B2, nucale avec périphériques 1 et 2 gauches et droites et pleurale 1 gauche partielle, faces dorsale et ventrale ; B3, B4, B5, plastron avec périphériques 6 à 8 gauches et 5 et 6 droites partielles, faces dorsale, ventrale et latérale gauche ; B6, pygale, faces dorsale et ventrale. Échelle : $1 \mathrm{~cm}$. 
verticales et ne sont pas redressées extérieurement à leur extrémité libre. Plusieurs plaques isolées appartiennent à des jeunes: elles sont relativement plus courtes et plus minces (Fig. 5N) comme chez $\mathrm{AaO}-949$. De la partie postérieure, il manque la ou les suprapygales. D'après les pleurales 8 préservées et les pygales, la ou les suprapygales formaient un bloc trapézoïdal à bord postérieur transversal. La pygale est conservée en plusieurs exemplaires ainsi que les périphériques postérieures, du pont à la 11 e (Figs 2; 4). La pygale est trapézoïdale à grand côté antérieur (qui contactait la suprapygale) et était encadrée par les périphériques 11 formant un coin aigu médiopostérieurement contre elle; la pygale varie un peu dans ses proportions (Figs 2E-K; 4B6) : certaines pygales, attribuées aux mâles, sont plus courbées, plus larges et plus épaisses (Fig. 2I, K) que les autres (Fig. 2H). Les pygales sont allongées par rapport à la condition primitive de la famille: leur longueur atteignait celle des périphériques (Fig. 2E, J, K), jusqu'au bord postérieur de la suprapygale prolongeant le bord des pleurales 8 sur une même ligne; de sorte que, comme antérieurement entre plaques et sillons à partir de la 2e périphérique, la suture périphériques/pleurales-suprapygale coïncidait avec le sillon marginales/costales-vertébrale 5.

Écailles: les paratypes montrent la variabilité de l'écaille vertébrale 1 : ses bords latéraux sont en lyre sur l'holotype, divergents puis presque parallèles à l'avant chez $\mathrm{AaO}-948$ et à peine arrondis vers l'extrémité antérieure chez AaO-949. Le spécimen de la nucale isolée $\mathrm{AaO}-4788$ pouvait aussi avoir eu une vertébrale en lyre. Les bords de cette vertébrale ne couvrent pas les coins de la nucale chez l'holotype $\mathrm{AaO}-272$, et à peine chez $\mathrm{AaO}-949$ et les nucales isolées $\mathrm{AaO}-4788$ et $\mathrm{AaO}-687$, mais la vertébrale 1 les couvre complètement chez $\mathrm{AaO}$ 948. Les pygales ont une surface lisse, sans sillon médian ce qui indique la présence d'une supracaudale simple (marginales 12 fusionnées).

Plastron. Comme sur l'holotype, le lobe antérieur est plus étroit que le postérieur avec des angles axillaires relativement fermés; les épiplastrons sont tous allongés, à saillie gulaire nette, le bourrelet épiplastral dorsal est toujours légèrement concave. Un seul spécimen sur huit montre que le bourrelet épiplastral ne couvre pas toujours l'entoplastron (Fig. 5D). Les hypoplastrons forment tous charnière avec les xiphiplastrons comme chez l'holotype, qu'ils soient femelles (par ex. AaO-948 et AaO-949) ou mâles (par ex. AaO-672; Fig. 5L). Les hyoplastrons sont épaissis médialement aux processus axillaires et les hypoplastrons et xiphiplastrons sont épaissis latéralement au maximum dans leur zone de contact, à la limite médiale du sillon des écailles fémorales. Tous les hypoplastrons participent un peu au lobe postérieur en avant des xiphiplastrons. Le lobe postérieur est large et ses bords sont arrondis jusqu'à la pointe xiphiplastrale. Les xiphiplastrons sont aussi larges que longs ou un peu plus larges (Fig. 5M), resserrés un peu ou non au sillon fémoro-anal, mais devenant plus convergents vers la pointe. Ils sont aussi épaissis au maximum à l'avant de leur pointe xiphiplastrale, au sillon médial des écailles anales. L'encoche anale est triangulaire, plus large que longue mais relativement profonde (Figs 4A1, A2, B3, B4; 5M); seuls trois spécimens sont connus par leur xiphiplastron dont les deux présumés femelles (AaO-948 et AaO-949) et le troisième (AaO-688, Fig. 5M) n'est pas affirmé comme mâle ou femelle, ne permettant pas de déceler un éventuel dimorphisme sexuel.

Écailles: les gulaires atteignent juste l'entoplastron ou le recouvrent un peu dorsalement. Elles s'élargissent à peine vers l'arrière sur le bourrelet

FIG. 5. - Testudo oughlamensis n. sp., Ahl al Oughlam, Maroc, Pliocène supérieur, coll. INSAP: A, AaO-675, épiplastron droit-entoplastron, faces ventrale et dorsale ; B, AaO-2127, épiplastron gauche, faces dorsale, ventrale et médiale (symphyse) ; C, AaO-674, épiplastron gauche-entoplastron, faces dorsale et ventrale ; D, AaO-673, épiplastron droit-entoplastron, faces ventrale et dorsale ; $\mathbf{E}, \mathrm{AaO}-672$, épiplastron gauche, faces dorsale, ventrale et médiale (symphyse); $\mathbf{F}, \mathrm{AaO}-4790$, hyoplastron droit, faces ventrale et dorsale ; G, AaO-4789, hyoplastron gauche, faces dorsale et ventrale ; $\mathbf{H}, \mathrm{AaO}-683$, hypoplastron gauche (partiel), faces dorsale et ventrale ; I, AaO-684, hypoplastron droit (partiel), faces ventrale et dorsale ; J, AaO-681, hypoplastron gauche (partiel), faces dorsale et ventrale ; K, AaO-685, hypoplastron gauche (partiel) faces dorsale et ventrale ; L, AaO-672 ; L1, L2, L3, hypoplastron droit, faces ventrale, dorsale et postérieure (charnière) ; $\mathbf{M}, \mathrm{AaO}-688$, xiphiplastron gauche, faces dorsale et ventrale ; $\mathbf{N}$, AaO-2121, périphérique du pont jeune, 5e-6e gauche, faces dorsale, ventrale et postérieure ; $\mathbf{O}, \mathrm{AaO}-2129$ et 2130 ; $\mathbf{0 1}, \mathbf{0 2}$, O3, périphériques 9 et 10 gauches, faces dorsales, postérieure et ventrale. Échelles : $1 \mathrm{~cm}$. 


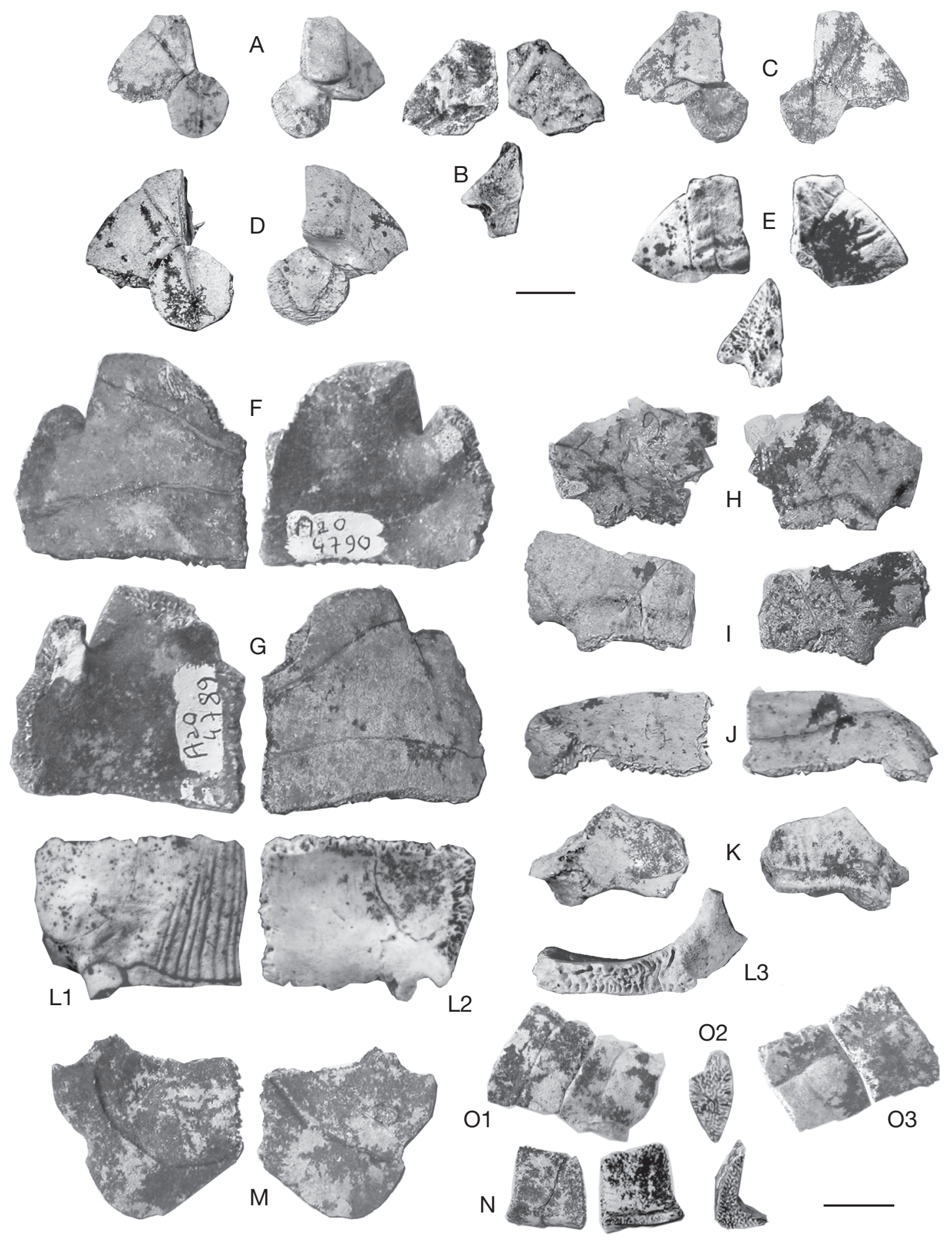


épiplastral dorsal comme chez l'holotype. Il y a une écaille axillaire triangulaire, allongée à cheval sur la périphérique 3 et l'hyoplastron, visible sur $\mathrm{AaO}-948$ ventralement. Le sillon huméro-pectoral contourne toujours l'entoplastron sans s'en approcher, avec une légère sinuosité convexe latérale et médialement en arrondi ou plus directement. La sinuosité plus ou moins latérale ou médiale du sillon pectoro-abdominal sur l'hyoplastron est faible. La sinuosité du sillon abdomino-fémoral est variable, de faible (sillon presque parallèle à la charnière hypo-xiphiplastrale) à forte, le sillon s'approchant le plus fortement de la suture médialement dans un seul cas sur 12 (Fig. 5L1) et restant séparé de la suture latéralement en vue ventrale; il rejoint ou non la suture dorso-latéralement (non visible ventralement). Les anales s'étendent médialement vers l'avant vers la suture hypo-xiphiplastrale, le sillon fémoro-anal restant à peu près parallèle au bord de l'encoche anale, laquelle est relativement profonde. La longueur des anales sur le xiphiplastron est plus grande que la longueur des abdominales sur l'avant des xiphiplastrons comme aussi sur l'arrière de l'hypoplastron. Il y a deux écailles inguinales, visibles ventralement sur certains spécimens dont $\mathrm{AaO}-948$, une petite hypoplastrale et une plus grande plus facilement visible, à cheval sur la périphérique 7 et l'hypoplastron, ventralement; elles sont séparées ventralement, se joignant dorsalement.

Humérus, AaO-696 (Fig. 6A). Il est incomplet distalement et aux trochanters. La diaphyse est recourbée, les trochanters sont écartés par une fosse intertrochantérique en $U$ plus large que chaque trochanter. La tête est arrondie.

\section{COMPARAISON}

La détermination de la nouvelle espèce de Testudo d'Ahl al Oughlam et des spécimens décrits plus loin est basée sur leur comparaison avec les espèces actuelles de Testudo, les spécimens du PléistocèneHolocène du Maroc déjà décrits (Gmira 1995) dont l'espèce T. kenitrensis Gmira, 1993, du Pléistocène moyen de Kénitra (au nord de Rabat) et les autres restes de Testudo fossiles du Maghreb (Lapparent de Broin 2000a). Les tortues fossiles du Maroc attribuées à l'espèce actuelle $T$. graeca (s.s.) com- portent les spécimens décrits in Gmira (1995) en tant que Testudo graeca graeca. Depuis l'étude de Gmira (1995) de nouveaux travaux modifient les rapports entre les espèces du genre et les rapports entre les genres de la sous-famille. Les études cladistiques menées à ce jour (Gmira 1995; Lapparent de Broin et al. 2006b, c) ne sont pas concluantes pour les relations phylétiques précises des genres distingués au sein d'un Testudo s.l. paléarctique subdivisé, du fait de l'insuffisance de caractères fournis pas les carapaces osseuses seules et de la grande quantité d'homoplasies possibles connues. Elles ont cependant permis de mieux apprécier les différences entre les genres correspondant aux subdivisions reconnues. Conformément à ces études, le genre Testudo est limité ici aux petites espèces paléarctiques à charnière hypo-xiphiplastrale chez les deux sexes et à bourrelet épiplastral élevé. Sont formellement exclues du genre Testudo les espèces sans charnière dans les deux sexes appartenant: 1) au genre paléarctique oriental Agrionemys Khozatsky \& Mlynarski, 1966; 2) au genre fossile Paleotestudo Lapparent de Broin, 2000, au sens large, en incluant le groupe promarginata (Broin, 1977) outre l'espèce type canetotiana Lartet, 1851 et $P$. mellingi (Peters, 1869) (voir Lartet 1851; Peters 1869); et 3) au genre "Eurotestudo" in Lapparent de Broin et al. 2006a-c (espèces fossiles et actuelles) i.e. Chersine Merrem, 1820, espèce type Testudo hermanni Gmelin, 1789, not Chersina Gray, 1831 (Gray 1831a, b) espèce type Testudo angulata Schweigger, 1812. Il a été montré que (Bour \& Ohler 2008), n'étant pas citée dans la publication originale du genre Medaestia Wussow, 1916, l'espèce Testudo hermanni ne pouvait être attribuée à ce dernier genre (contrairement à l'une des propositions de Fritz \& Kraus 2008) mais seulement à Chersine. Il a été prouvé que les lignées Chersine ("Eurotestudo" in Lapparent de Broin et al. 2006a-c), Agrionemys et Testudo sont distinctes depuis le Miocène supérieur, Vallésien, MN 10, au moins (Lapparent de Broin et al. 2006b, c; Garcia et al. 2011). Au sein du genre Testudo s.l., les divisions et regroupements en espèces ou sous-espèces actuelles sont basées souvent sur des caractères dont beaucoup proviennent de la taille et de la morphologie générale, mais surtout des écailles et des couleurs de la tête, de la carapace et des membres, de sorte que 
les caractères ne sont pas conservés chez les fossiles; bien de ces caractères sont souvent reconnaissables seulement par une étude statistique avec un échantillon important (Perälä 2002a, b; Pieh \& Perälä 2004 ; Lapparent de Broin et al. 2006b, c). Dès les premières études (Gmira 1992, 1993a, b, 1995) a été montrée la nécessité d'avoir, en ce qui concerne les fossiles comme les actuels, un échantillon suffisant de squelettes, ce qui n'est pas souvent le cas. Le groupe T. graeca s.s. est considéré ici comme incluant les sous-espèces actuelles du Maghreb, s'étendant du Maroc à la Libye: Testudo g. graeca Linnaeus, 1758; T. g. cyrenaica Pieh \& Perälä, 2002; T. g. soussensis Pieh, 2000, T.g. marokkensis Pieh \& Perälä, 2004; T. g. lamberti Pieh \& Perälä, 2004; T. g. nabeulensis Highfield, 1990. Les spécimens fossiles du Maroc attribués à Testudo graeca sont déterminés à l'aide des squelettes de spécimens actuels (Algérie, Maroc) des collections du MNHN, dont ceux récoltés dans les régions de Fez et de Rabat; pour ceux-ci, la sous-espèce possible serait $T . g$. marokkensis mais les squelettes à notre disposition ne permettent pas vraiment d'attribution précise en regard des définitions des sous-espèces sur les caractères externes.

Chez T. oughlamensis $n$. sp. la plupart des caractères sont généralisés et communs à plusieurs genres de Testudininae incluant Testudo et représentant plusieurs lignées dans le monde (Broin 1977; Lapparent de Broin 2000b, 2002, 2003, 2008; Lapparent de Broin et al. 2006b, c). Ces caractères généralisés sont soit présents à la base de la sous-famille, soit communs par synapomorphie ou par homoplasie chez certains genres à partir d'un certain grade évolutif, mais seule une étude cladistique complète des Testudinidae mettrait en évidence leur répartition : 1) la forme générale de la carapace (dossière et plastron, plaques et écailles); 2) la présence d'écailles axillaires et inguinales (primitive) ; 3) la forme générale de la plaque nucale; 4) la différentiation des neurales avec une première neurale quadrangulaire (primitivement elle est hexagonale à petits côtés postérieurs) précédant quelques plaques octogonales et carrées; la différenciation des neurales en plaques octogonales et carrées n'est pas stabilisée ici d'après l'holotype ; 5) la forme du plastron avec les gulaires triangulaires longues (primitivement elles sont très courtes); 6) la morphologie des étroits
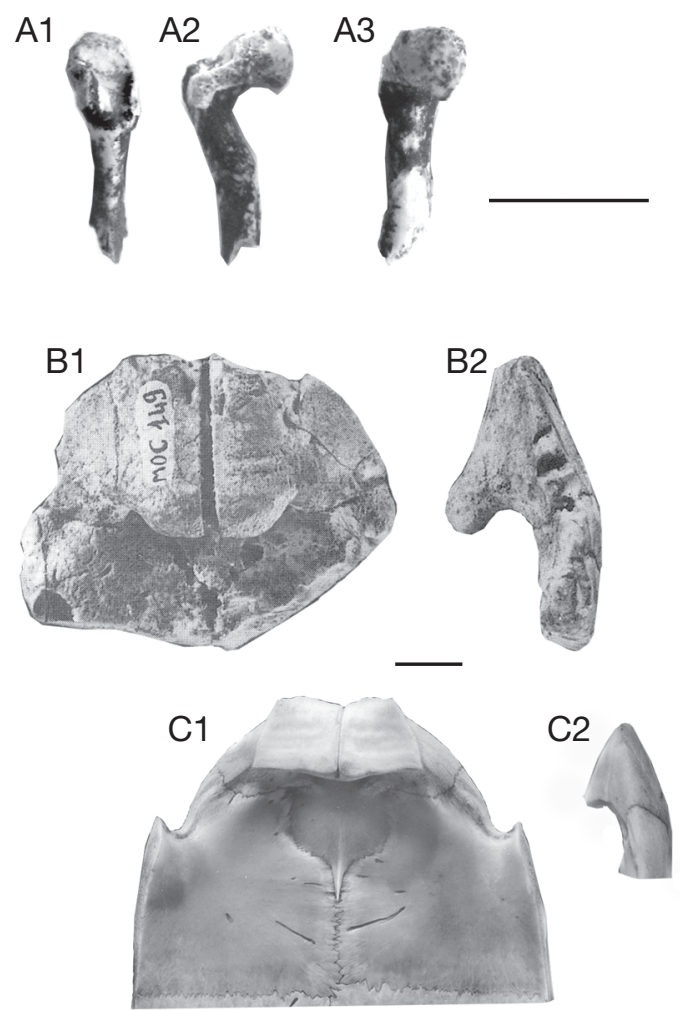

FlG. 6. - A, Testudo oughlamensis n. sp., Ahl al Oughlam, Maroc, Pliocène supérieur, coll. INSAP, AaO-696 ; A1, A2, A3, humérus droit partiel, faces ventrale, latéro-antérieure et dorsale ; B, Testudo kenitrensis Gmira, 1993, MNHN.F.MOC149, holotype, sommet du Pléistocène moyen (Inter Amirien-Tensiftien), Kenitra, Maroc ; B1, B2, lobe antérieur partiel, faces dorsale et latérale gauche; C, Testudo graeca Linnaeus, 1758, MNHN.F (coll. REP 27), Afrique du Nord (Algérie?) actuel ; C1, C2, Plastron, lobe antérieur, vues dorsale et latérale droite. Échelles : $1 \mathrm{~cm}$.

et courts contreforts plastraux dressés le long des périphériques du pont; 7) le lobe postérieur plus large que l'antérieur; et 8) le pont long.

La présence de pointes aux extrémités des sillons des marginales est un caractère représenté chez certains Testudininae dans les lignées paléarctiques dès l'Éocène supérieur et l'Oligocène dont «l'Ergilemys Čkhikvadze, 1972» de France (Broin 1977, 1978; Lapparent de Broin 2000b; Lapparent de Broin et al. 2006b, c) et certains petits endémiques africains actuels tels Homopus Duméril \& Bibron, 1834, et Kinixys Bell, 1827, ainsi que Namibchersus namaquensis (Stromer, 1926) du Miocène inférieur 
(Lapparent de Broin 2003, 2008), accompagnant éventuellement une encoche du bord antérieur. La coïncidence des sillons des écailles costales-vertébrale 5/marginales-supracaudale avec les sutures pleurales-suprapygales/périphériques-pygale sur le pourtour de la dossière est un caractère dérivé par rapport au stade du morphotype le plus primitif (celui de Manouria impressa (Günther, 1882)) et au stade du morphotype "Geochelone» (Lapparent de Broin 2003, 2008) : la pygale est élevée ici jusqu’au niveau des périphériques et sa suture proximale avec la suprapygale coïncide avec la limite postérieure de la vertébrale 5 . Ce caractère est notamment partagé par Paleotestudo, Chersine ("Eurotestudo» in Lapparent de Broin et al. 2006a-c), Testudo et "Testudo" semenensis Bergounioux, 1955 du Miocène supérieur de Djebel Sémène (Tableau 1) de Tunisie (voir Bergounioux 1955). Dans l'ancien monde, la configuration suprapygale - pygale de ces derniers est proche aussi de celle d'Agrionemys (mais avec une différentiation dans le raccourcissement médian de la vertébrale 5 chez ce dernier) et de celle des petits endémiques africains (mais avec d'autres particularités des suprapygales et du sillon de la vertébrale 5) (Gmira 1995; Lapparent de Broin 2003; Lapparent de Broin et al. 2006b, c). La fusion des marginales 12 en une supracaudale est apparue à partir de l'Éocène supérieur-Oligocène en Europe occidentale, comme chez l'oriental Agrionemys et aussi chez d'autres Testudininae; une redivision progressive de la supracaudale se produit aux Pliocène-Quaternaire chez Chersine, soit d'un seul côté, soit des deux côtés (dorsal et ventral) comme le plus souvent chez les spécimens actuels. La pygale de $T$. oughlamensis n. sp. est recourbée vers l'intérieur comme chez la plupart des espèces de Testudininae néogènes et au moins chez les mâles actuels, sauf chez T. marginata où la bordure postérieure s'étale plus largement y compris au niveau de la pygale chez les mâles comme chez les femelles.

T. oughlamensis $\mathrm{n}$. sp. et T. kenitrensis partagent avec la majorité des espèces de Testudininae (primitivement) la sinuosité latérale modérée du sillon pectoro-adominal. Toutefois T. marginata Schoepff, 1789 et T. kleinmanni Lortet, 1883 se distinguent des autres espèces de Testudo, y compris les fossiles du Maroc décrits ici, par l'infléchissement médian des pectorales beaucoup plus fort, celles-ci rejoignant presque la suture hyo-hypoplastrale; par ailleurs T. marginata et T. kleinmanni se distinguent l'une de l'autre par d'autres caractères absents chez les Testudininae du Maroc, dont la forte encoche nucale en $\mathrm{V}$ chez T. kleinmanni, et la longue carapace étroite et l'étalement de la bordure postérieure chez T. marginata (Lapparent de Broin et al. 2006b, c).

La petite taille de l'entoplastron est un caractère dérivé commun à Paleotestudo, Testudo et Chersine, et parfois aussi à certains endémiques africains où il a tendance à se réduire encore plus (Lapparent de Broin 2003, 2008).

Testudo oughlamensis $\mathrm{n}$. sp. et T. kenitrensis forment un clade avec les autres espèces de Testudo, défini principalement par la présence d'une charnière hypo-xiphiplastrale chez tous les individus (Lapparent de Broin et al. 2006b, c) avec de ce fait le raccourcissement des hypoplastrons à l'arrière, la proximité du sillon abdomino-fémoral et de la charnière médialement, avec la possibilité de leur contact tout à fait latéralement, dorsalement au moins. La présence d'une charnière hypo-xiphiplastrale existe aussi chez des spécimens isolés d'espèces de Testudininae indéterminées de l'Oligocène et de l'Aquitanien de France attribuées avec doute à "Ergilemys» sp., avec une conjonction différente de caractères des éléments isolés dont la pygale large et courte et les marginales 12 divisées (en partie au moins) primitivement d'une part, les xiphiplastrons atteignant les encoches inguinales (seule participation du xiphiplastron au lobe postérieur (Broin 1977: pl. 25, figs 11, 12), ce qui est plutôt rare chez Testudo) et le bourrelet épiplastral long et aplati apomorphiquement d'autre part (Broin 1977, 1978 ; Lapparent de Broin et al. 2006b, c).

Testudo oughlamensis $\mathrm{n}$. sp. et T. kenitrensis diffèrent des espèces de Testudo actuelles par deux caractères primitifs: 1) la bordure périphérique postérieure ne s'étale pas «en une jupette» qui serait visible dorsalement chez T. kenitrensis et, en l'absence de celles de l'holotype de T. oughlamensis n. sp., les périphériques postérieures isolées (paratypes) bien verticales indiquent qu'il en allait de même dans cette dernière espèce. Il en est de même chez Paleotestudo. Une éventuelle différentiation sexuelle chez les espèces actuelles 
du Maghreb dans l'importance de l'étalement n'est pas évidente sur le squelette osseux: dans l'ensemble une fois les écailles enlevées, les spécimens adultes de T. graeca du Maroc examinés, et plus précisément ceux de Rabat à Fez, ont un bord postérieur osseux variable d'à peine redressé postérieurement aux périphériques 10 et 11 à nettement étalé aux périphériques 11 et redressé à la pygale chez la femelle (pygale bombée mais aussi visible dorsalement chez le mâle); cet étalement est visible dorsalement, par exemple chez les spécimens de $T$. graeca du Pléistocène supérieur du Maroc (Gmira 1995: pl. VIIA) et chez la plupart des autres espèces actuelles de Testudo avec une grande variabilité suivant celles-ci : étalement postérieur beaucoup moins marqué chez un petit spécimen de Testudo cyrenaica de Libye femelle que chez un grand spécimen femelle de T. ibera de Turquie par exemple; un extrême étalement, très dérivé, se produit chez $T$. marginata mâle et femelle; 2) La participation primitive de l'hypoplastron au lobe postérieur est constante dans le clade kenitrensis, le sillon abdomino-fémoral, sinueux, restant séparé de la charnière sur la face ventrale, latéralement et médialement; cette participation tend à disparaître totalement chez les spécimens actuels. Chez de nombreux spécimens actuels de T. graeca du Maroc (dont REP 21, coll. en dépôt au MNHN.F, $17 \mathrm{~cm}$ de long) et d'autres d'espèces plus orientales (T. ibera, T. marginata), le sillon abdomino-fémoral contacte largement la suture médialement (Lapparent de Broin 2002: fig. 19) et se confond souvent presque totalement avec la charnière; l'hypoplastron est raccourci jusqu'à ce qu'il ne participe pratiquement plus au lobe postérieur et que le xiphiplastron atteigne le fond de l'encoche inguinale. Pour ce dernier cas, il en est de même chez les éléments isolés attribués à "Ergilemys» (Broin 1977: pl. 25, fig. 11; pl. 28, fig. 7) (voir ci-dessus).

La taille réduite est dérivée mais elle n'est pas unique aux espèces fossiles marocaines: T. oughlamensis $\mathrm{n}$. sp. comme T. kenitrensis sont d'une petite taille, plus petite mais assez proche de celle de la population actuelle du NW et du centre du Maroc, T. g. marokkensis Pieh \& Perälä, 2004. La taille est encore plus petite chez T. kleinmanni d'Égypte.
L'angle des encoches axillaires est variable chez les actuels Testudo, variant d'aussi fermé dans le clade kenitrensis que chez $T$. graeca à plus ouvert (T. kleinmanni), comme chez Mauremys le géoémydiné présent aussi à Ahl al Oughlam.

Testudo oughlamensis $\mathrm{n}$. sp. et T. kenitrensis forment un clade kenitrensis qui diffère de toutes les autres espèces de Testudo par la présence du bourrelet épiplastral élevé et en corniche mais à surface dorsale légèrement concave. Chez les autres espèces de Testudo, ce bourrelet est aussi élevé mais à surface convexe comme chez d'autres Testudininae (Lapparent de Broin et al. 2006b: fig. 3) et il est plus court par rapport à la largeur de la saillie gulaire chez les espèces actuelles. Ce caractère de la concavité du bourrelet élevé est primordial pour caractériser le clade. Il est considéré ici comme un caractère dérivé à partir du stade à bourrelet convexe de Testudo considéré comme plus primitif: toutefois celui-ci est lui-même dérivé pour les Testudinidae. Le bourrelet convexe est plus développé que dans les stades de base à bourrelet non élevé et largement concave tel celui préservé chez l'actuel Manouria impresssa (Günther, 1882) (Lapparent de Broin et al. 2006b: fig. 3a) et encore proche de celui des Geoemydinae, et tel celui un peu plus dérivé du genre indéterminé à ce jour, représenté par "Achilemys» cassouleti Claude \& Tong, 2004 et "Hadrianus" sp. in Broin (1977) de l'Éocène inférieur de France (voir Claude \& Tong 2004), génériquement nommés de façon inappropriée. Ce stade est aussi différemment développé du long bourrelet aplati des "Ergilemys» de France (Broin 1977: figs 109, 110; pl. 27: fig. 11; pl. 29). Le stade convexe de Testudo est partagé avec les genres occidentaux Chersine ("Eurotestudo» in Lapparent de Broin et al. 2006a-c) (Pliocène-Actuel) et Paleotestudo, avec l'oriental Agrionemys et indépendamment (avec des variations dans les proportions) avec d'autres genres de Testudininae comme par exemple en Afrique dans d'autres lignées telle celle de Stigmochelys Gray, 1873 (Lapparent de Broin 2003, 2006b). À la différence de Testudo, tous ces genres sont tous toujours dépourvus de charnière plastrale (au moins chez les mâles car les femelles adultes de Chersine hermanni hermanni peuvent en avoir une, facilitant l'oviposition). Mais chez Cher- 
sine et Agrionemys l'extension du bourrelet ne va pas jusqu'au-dessus de l'entoplastron, la poche gulaire est antérieure à l'entoplastron et faible quand elle existe chez Chersine (dans les populations de l'espèce Chersine hermanni du Quaternaire de Lunel-Viel et de Soave) et il y a toujours l'absence de charnière et la division secondaire de la supracaudale ainsi que d'autres particularités pour distinguer le groupe hermanni; tandis que d'autres caractères dont la forme du lobe antérieur à grand entoplastron et la grande hauteur du pont sont propres à Agrionemys (Broin 1977; Lapparent de Broin et al. 2006b, c). Toutefois chez «T. graeca terrestris Forskål, 1775", in Gmira (1995) (dont REP 50, coll. en dépôt au MNHN.F) le bourrelet épiplastral, sans poche gulaire, s'arrête en avant de l'entoplastron; ce spécimen de Testudo est alors morphologiquement proche sur ce seul point des Chersine quaternaires et d'Agrionemys, sa charnière hypo-xiphiplastrale étant présente pour le distinguer, entre autres différences.

D'autres caractères diagnostiquent $T$. kenitrensis et T. oughlamensis $\mathrm{n}$. sp. mais comme leur présence se manifeste éventuellement chez d'autres spécimens pliocènes de Testudo des environs d'Ahl Al Oughlam dépourvus de l'apomorphie du bourrelet concave, ils peuvent caractériser un groupe kenitrensis plus large que le clade:

1) L'encoche nucale trapézoïdale profonde du bord antérieur de la dossière, visible au moins dorsalement, avec nucale en retrait par rapport aux premières périphériques. Si les bords médiaux des périphériques 1 sont encochés, concaves, sur les paratypes de T. oughlamensis n. sp., ils apparaissent plus rectilignes chez l'holotype comme chez T. kenitrensis; mais comme ils sont un peu érodés au bord, on ne saurait affirmer qu'ils n'aient pas été également un peu encochés. Une encoche trapézoïdale semblable à celle du clade kenitrensis, forte et visible dorsalement, existe chez le spécimen de Testudo sp. B d'Ar Rehamna décrit ci-dessous et permet de l'associer dans le groupe kenitrensis. Cette encoche nucale trapézoïdale du bord antérieur de la dossière osseuse dans le clade kenitrensis est l'accentuation profonde de la légère concavité du bord antérieur de la carapace dorsale, souvent présente chez les autres espèces de Testudo y compris T. graeca (spécimens MNHN.F en provenance d'Oran [Algérie] REP 30, ou de Rabat
[Maroc] REP 20, par exemple); elle est visible chez les spécimens actuels quand les écailles sont enlevées pour montrer le bord osseux, lequel ne correspond pas toujours avec le bord écailleux (croissance différentielle de l'os dermique et de l'écaille sur les pourtours). Cette concavité très modérée du bord antérieur est d'ailleurs présente chez certains spécimens de Testudo graeca du Pléistocène supérieur du Maroc: Tensiftien de la petite dune de Rabat à Temara (Gmira 1995: pl. VIB) par exemple. Une faible encoche nucale trapézoïdale très large, non visible dorsalement, existe chez les actuels comme le spécimen de T. terrestris cité ci-dessus (MNHN.F, REP 50) et chez un spécimen de T. ibera Pallas, 1814 de Turquie (REP 73) notamment. Une forte encoche nucale visible dorsalement existe, mais en $\mathrm{V}$ profond dont les branches partent rectilignes de chaque côté de la cervicale, chez T. kleinmanni et chez Testudo sp. du Pliocène de Çalta (Turquie) (Rage \& Sen 1976) et de Grèce (Garcia et al. 2011) avec un allongement très fort des périphériques encadrantes 1 et 2 . Il y a donc une tendance à l'approfondissement de l'encoche du bord antérieur chez Testudo, faible au départ et soit en trapèze (à son maximum dans le groupe kenitrensis), soit en $\mathrm{V}$ (à son maximum dans l'espèce de Çalta). L'encoche trapézoïdale du bord antérieur de la carapace du clade T. kenitrensis-T. oughlamensis n. sp. rappelle les actuels Kinixys et Homopus d'Afrique (carapace osseuse), avec la cervicale saillante ou non suivant les espèces comme ici dans le groupe marocain; elle rappelle aussi le jeune de Namibchersus namaquensis, l'encoche étant plus large antérieurement et s'amoindrissant chez l'adulte (Lapparent de Broin 2003, 2008). Elle rappelle aussi «Ergilemys» bruneti Broin, 1977 de l'Oligocène de France, avec aussi des pointes périphériques à la jonction des marginales et une saillie médiane de la nucale, toutefois moins en retrait des périphériques 1 . En tout état de cause, les autres écailles et plaques du groupe kenitrensis n'ont pas les mêmes proportions chez Kinixys, Homopus, Namibchersus et "E." bruneti.

2) La forme de la vertébrale 1 qui peut être en forme de lyre chez plusieurs individus: deux spécimens de T. kenitrensis et l'holotype de T. oughlamensis n. sp., au moins. Tous les spécimens de $T$. oughlamensis n. sp. n'ont pas la vertébrale 1 en lyre comme ceux 
de T. kenitrensis. Comme il n'y a que deux spécimens connus de T. kenitrensis, la constance de la lyre au sein de cette espèce n'est peut-être pas absolue. Quoiqu'il en soit ce sont les seules espèces nommées présentant éventuellement une telle lyre en commun avec les autres caractères chez Testudo et, constant ou non, le caractère peut faire partie de la diagnose d'un groupe kenitrensis plus large que le clade; en effet, le spécimen de Testudo sp. B d'Ar Rehamna décrit ci-dessous a aussi une vertébrale 1 presque en lyre; d'autre part, un spécimen de Testudo sp. $\mathrm{A}$, de Dehar Mouak décrit ci-dessous, du Pliocène un peu inférieur à celui de T. oughlamensis n. sp., possède également une vertébrale 1 en lyre, de sorte qu'il est rapproché du groupe T. kenitrensis par ce seul caractère. Chez les autres espèces de Testudo, la vertébrale 1 a les bords latéraux arrondis (le plus souvent chez les T. graeca du Maroc et d'Algérie comme souvent chez T. ibera) sans le net resserrement assez long pour caractériser la forme de lyre (coll. REP, en dépôt au MNHN.F); ou bien ces espèces ont des bords rectilignes comme chez un spécimen d'Ahl al Oughlam (Fig. 4) et, souvent, chez les jeunes spécimens actuels de Testudo, atteignant les bords latéraux de la nucale ou recoupant plus ou moins ces bords comme les autres spécimens d'Ahl al Oughlam (Fig. 4); ou bien les bords latéraux de la vertébrale 1 dépassent largement la nucale, comme chez un spécimen au moins de T. cyrenaica Pieh \& Perälä, 2002 (MNHN.F, REP 24).

3) La forme, large pour la longueur, courte et bien arrondie, de la dossière de T. kenitrensis et T. oughlamensis $\mathrm{n}$. sp. (non totalement conservée à l'arrière chez l'holotype mais complétée par les paratypes). Les parties antérieure et postérieure de la dossière sont inclinées vers le bas (Fig. 2A4; Gmira 1995: pl. II) sans un long plateau horizontal entre elles comme il y en a chez les autres espèces de Testudo dont T. graeca (Fig. 6C; Gmira 1995: pls VIIB, IXC) et la petite espèce T. kleinmanni. Il en est de même pour le spécimen de Testudo sp. B d'Ar Rehamna et le caractère est aussi attribué de ce fait au groupe kenitrensis.

4) Les vertébrales sont moins larges que chez T. graeca dans les deux espèces, d'après le rapport largeur sur longueur de la vertébrale 3, de $142 \%$ chez T. oughlamensis n. sp. et de $138 \%$ chez T. kenit- rensis pour c. 152 à $155 \%$ chez T. graeca, mâles et femelles; la série vertébrale est encore plus étroite dans l'espèce Testudo sp. B d'Ar Rehamna (rapport de $114 \%$ pour la vertébrale 3) rapportée de ce fait aussi au groupe kenitrensis.

T. kenitrensis et T. oughlamensis n. sp. diffèrent en outre de $T$. graeca par des anales plus longues médialement que chez $T$. graeca, pointant vers la suture hypo-xiphiplastrale de sorte que médialement leur longueur est nettement bien plus grande que la longueur des fémorales, comme par ailleurs chez T. kleinmanni et T. cyrenaica. Ce caractère n'étant pas connu chez l'espèce Testudo sp. B d'Ar Rehamna est susceptible de caractériser tout le groupe en conjonction avec les autres caractères.

T. oughlamensis n. sp. et T. kenitrensis diffèrent l'un de l'autre par:

1) le bord antérieur de la nucale (préservée chez cinq spécimens) avec une saillie médiale chez T. oughlamensis $\mathrm{n}$. sp. : la saillie affecte toute la plaque médialement et pas seulement la partie recouverte par l'écaille cervicale (5 spécimens); la plaque a son bord antérieur rectiligne chez T. kenitrensis (deux spécimens);

2) le bourrelet dorsal moins long sur l'entoplastron chez T. oughlamensis n. sp. (Figs $2 ; 4 ; 5$ ) que chez T. kenitrensis (Fig. 6B), ce qui est vrai pour les 8 lots d'épiplastrons (les 3 paires des trois carapaces et 5 isolés) de T. oughlamensis n. sp. En revanche chez T. oughlamensis n. sp. le lobe antérieur est relativement plus long: les épiplastrons qui forment le bourrelet sont un peu allongés vers l'avant médialement, avec des gulaires relativement un peu plus étroites pour leur longueur;

3) l'insertion du contrefort hypoplastral, mais c'est un caractère potentiellement variable intraspécifiquement; elle se fait principalement sous la périphérique 7 avec un petit débordement ventral sous la périphérique 8 chez T. oughlamensis n. sp. (comme chez un T. ibera de Turquie et chez certains T. marginata de Grèce); alors qu' elle se fait complètement entre les deux plaques chez T. kenitrensis (visible sur l'holotype seul) comme généralement chez Testudo, où l'insertion peut aussi se faire: sous la $7 \mathrm{e}$ seule (chez certains $T$. graeca d'Afrique du Nord); sous la périphérique 7 avec un petit débordement dorsal sous la périphérique 8 (chez quelques T. graeca du 
Maroc et $T$. terrestris); et sous la $8^{\mathrm{e}}$ principalement (chez un T. graeca d'Afrique du Nord);

4) les écailles vertébrales 2, 3 et 4, relativement plus étroites (largeur par rapport à la longueur de chaque écaille et particulièrement la 3 e) distinguent l'holotype de T. kenitrensis de celui de T. oughlamensis n. sp. à vertébrales un peu plus larges; l'amplitude de la variabilité (compte tenu du sexe notamment) ne peut être établie mais ce qui reste des spécimens $\mathrm{AaO}-948$ et AaO-949 (Fig. 4) confirme l'holotype (Fig. 3), tous trois devant être des femelles comme T. kenitrensis d'après le plastron plat;

5) il n'y a qu'une grande écaille inguinale chez T. kenitrensis au lieu de deux petites chez T. oughlamensis n. sp. (et chez Testudo graeca il y en a deux dont l'externe plus grande).

\section{?Groupe kenitrensis}

Testudo sp. A

(Fig. 7A1, A2)

MATÉRIEL . — Une dossière seule, T CDI1, coll. INSAP.

LOCALITÉ ET HORIZON STRATIGRAPHIQUE. Environs de Casablanca, Maroc, cordons dunaires de la côte de Casablanca à Kenitra, Maroc (voir ci-dessus). Pliocène (non défini).

\section{DESCRIPTION}

La carapace a une forme allongée avec un relativement long plateau dorsal sous les vertébrales 2 et 3 et l'avant de la 4e. Les sillons des écailles costalesmarginales coïncident avec les sutures pleurales-périphériques. Les annuli de croissance sont nombreux et bien marqués et le disque pleural est bosselé sous les écailles, traduisant un état bien adulte. Le bord antérieur a une faible encoche large, à peine visible dorsalement, affectant un peu la périphérique 1 gauche et la nucale à gauche. La série des neurales (n) 2 à 5 est différenciée (n2 octogonale, n3 quadrangulaire, $\mathrm{n} 4$ octogonale et $\mathrm{n} 5$ quadrangulaire) les suivantes sont hexagonales. Les suprapygales 1 et 2 sont séparées par une suture transversale convexe, la première embrassant l'avant de la seconde. La pygale est large, à bords latéraux arrondis, haute jusqu'au sillon postérieur de la vertébrale 5 et convexe. Les bordures périphériques antérieure et postérieure ne sont pas relevées en une "jupette» visible dorsalement, les périphériques postérieures étant bien droites suivant la pente du disque pleural à gauche, mais la bordure est un peu disloquée et artificiellement relevée à droite. La cervicale est étroite et s'étrécit vers l'avant. Les vertébrales sont plutôt larges (vertébrale 3 : largeur sur longueur de $155 \%$ ). La vertébrale 1 a ses bords arrondis et en lyre, surtout à droite, laissant les costales 1 mordre largement sur les coins postérieurs de la nucale.

\section{COMPARAISON}

La forme longue avec un long plateau longitudinal médian, est celle primitive pour les Testudininae dont Testudo graeca. La différentiation neurale est conforme aux Testudo actuels et de même la série vertébrale qui est relativement large (primitivement). L'encoche antérieure n'est pas assez prononcée pour être celle, dérivée, du groupe kenitrensis et elle a son équivalent chez Testudo graeca, de même que la largeur des vertébrales. Les bordures périphériques antérieure et postérieure non relevées représentent un caractère primitif par rapport à $T$. graeca tout en la rapprochant du groupe kenitrensis. La forme de la vertébrale 1 est en lyre comme cela se produit dans le groupe kenitrensis. En l'absence de plastron il est difficile de définir une espèce nouvelle, et de voir si elle représentait une population du Pliocène inférieur dérivée par rapport à la forme primitive dans le sens ou non du groupe kenitrensis: comme, par opposition à $T$. graeca, elle partage le caractère dérivé de la vertébrale en lyre avec le groupe kenitrensis, elle en est provisoirement rapprochée.

\section{Groupe kenitrensis}

Testudo sp. B, aff. kenitrensis Gmira, 1993

(Fig. 7B1-B4)

MATÉRIEL. — Carapace T AR1, coll. INSAP, comprenant la dossière avec la bordure périphérique antérieure partielle, de la périphérique 6 gauche à la périphérique 4 droite en incluant la nucale, et avec une partie du plastron: lobe antérieur avec tout l'hyoplastron gauche, l'entoplastron et les épiplastrons outre la partie antérieure de l'hypoplastron droit. 

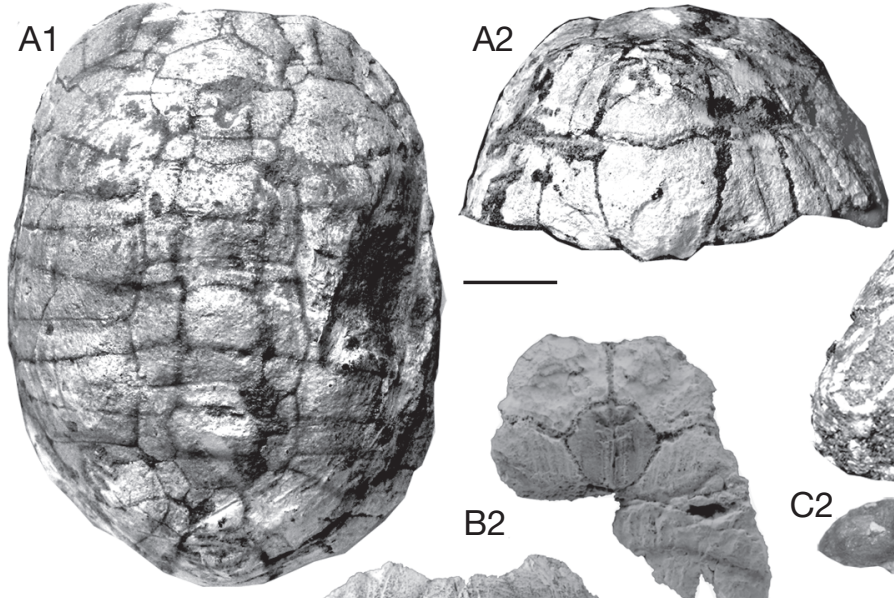

\section{C1}
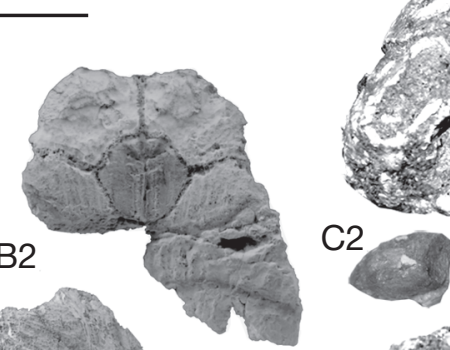

C2

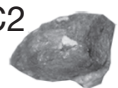

B1
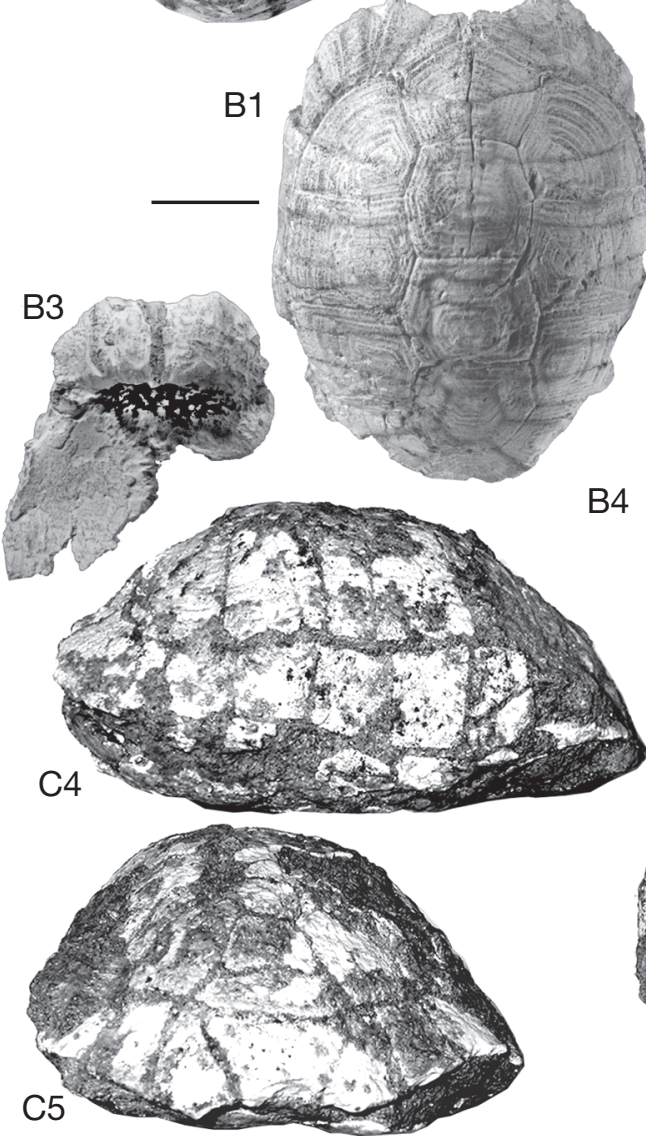

B4

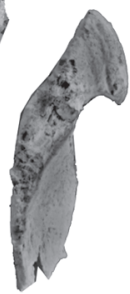

C3

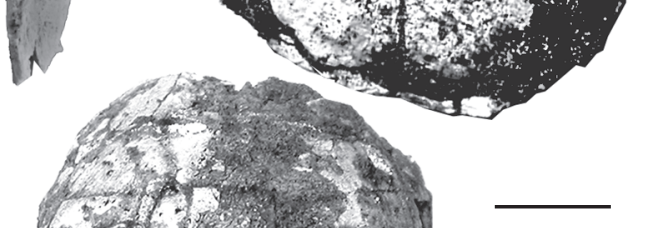

的
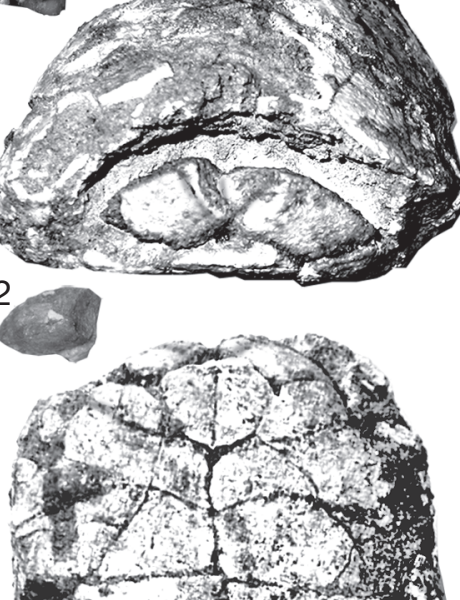

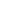


LOCALITÉ ET HORIZON STRATIGRAPHIQUE. Carrière d'Ar Rehamna, près de Casablanca, Maroc, du complexe de cordons dunaires de la côte de Casablanca à Kenitra, Maroc, entaillée dans le cordon d'Al Hrawiyine (Fig. 1). Unité morpho-sédimentaire de Sidi Messaoud, couche $\mathrm{C} 1$ sous la Dune D1b. Carte géologique, feuille de Casablanca-Mohammedia (Destombes, 1987). Piocène supérieur, c. 2,5 Ma, semble-t-il légèrement plus récent que Ahl al Oughlam (voir ci-dessus).

\section{DESCRIPTION}

La dossière est courte pour sa largeur et son profil en vue latérale est très arrondi sans long plateau médian. Les annuli de croissance sont très marqués et les sutures difficilement visibles, montrant qu'il s'agit d'un adulte avéré. La bordure périphérique antérieure n'est pas relevée, la postérieure faisant défaut. Les sillons des écailles costales-marginales coïncident avec les sutures pleurales-périphériques. Le bord antérieur de la dossière porte une profonde encoche trapézoïdale: la périphérique 1 droite a un bord médial concave, faisant une petite pointe arrondie à son extrémité antérieure au sillon des marginales 1-2; la nucale est bien en retrait par rapport aux périphériques 1 et avec une très légère saillie médiane où la cervicale vient s'effiler au milieu. La série des neurales est régulièrement différenciée à l'avant (n2 octogonale, $\mathrm{n} 3$ quadrangulaire, $\mathrm{n} 4$ octogonale); les neurales 5 et 6 sont hexagonales, la $6 \mathrm{e}$ semblant fusionnée avec la $7 \mathrm{e}$, et la $8 \mathrm{e}$ ne se distingue pas des suprapygales en formant un trapèze. Le bord postérieur du trapèze est transversal et aligné sur le bord des pleurales, indiquant une pygale élevée à ce bord, coïncidant avec le bord postérieur de la vertébrale 5. La vertébrale 1 a des bords latéraux un peu arrondis, resserrés puis divergents vers l'avant, amorçant une forme de lyre, en ne recouvrant pas les petits coins latéro-postérieurs de la nucale (couverts par les costales 1). La série vertébrale est relativement étroite (vertébrale 3: largeur sur longueur de $114 \%$ ) même par rapport à T. kenitrensis (V3: largeur sur longueur de $138 \%$, contre c. $152-155 \%$ chez $T$. graeca). Le lobe antérieur a une forme rare pour un Testudo, en un trapèze large à l'avant à bords latéraux parallèles puis convergents vers l'avant et avec un large bord antérieur un peu incurvé. De ce fait, les gulaires sont larges à l'avant; elles mordent un peu sur l'entoplastron à l'arrière.
Le sillon huméro-pectoral fait un angle près du bord latéral et vient passer assez près en arrière de l'entoplastron. Dorsalement, le bourrelet épiplastral, épais et convexe, est large avec des gulaires larges et longues, long jusqu’à l'entoplastron.

\section{COMPARAISON}

La dossière est courte et très arrondie dorsalement sans long plateau médian comme chez le clade kenitrensis. L'espèce peut faire partie du groupe kenitrensis par son encoche nucale (caractère dérivé) mais elle ressemble plus particulièrement à $T$. kenitrensis par le bord antérieur de la nucale peu saillant (caractère primitif) et la plus grande étroitesse des vertébrales (dérivé). Elle se rapproche aussi du groupe par l'amorce de lyre à la vertébrale 1 (dérivé). Elle s’en écarte radicalement par le lobe antérieur et le bourrelet épiplastral, non dérivé par une concavité comme chez le clade T. kenitrensis mais convexe (plus primitivement comme chez T. graeca) (Fig. 6C2) et autapomorphe par sa longueur et son arrondi marqué (Fig. 7B3, B4), avec des gulaires un peu plus étroites dorsalement (Fig. 7B3) que chez T. graeca (Fig. 6C1); elles sont plus longues que chez T. oughlamensis $\mathrm{n}$. sp. et plus courtes que celles de T. kenitrensis (Fig. 6B1). Le spécimen pourrait être une espèce nouvelle groupefrère de T. oughlamensis n. sp. - T. kenitrensis, dérivée pour sa part par son bourrelet soit à partir du bourrelet primitif soit à partir du bourrelet concave du clade kenitrensis; la vertébrale 1 ne forme pas une lyre parfaite par son élargissement antérieur au resserrement, mais presque, et tous les spécimens de T. oughlamensis $\mathrm{n}$. sp. n'ont pas une vertébrale en lyre, laquelle ne caractérise qu'une partie de l'échantillon.

\section{Testudo sp. C}

(Fig. 7C1-C6)

MATÉRIEL. - Une carapace complète, dossière et plastron (T DM1, coll. INSAP).

LOCALITÉ ET HORIZON STRATIGRAPHIQUE. — Dehar Mouak, près de Casablanca, Maroc, cordons dunaires de la côte de Casablanca à Kenitra, Maroc. Unité morpho-sédimentaire de Dehar Mouak (voir ci-dessus). Carte géologique, feuille de Casablanca-Mohammedia (Destombes, 1987). Pliocène inférieur. 


\section{DESCRIPTION}

La surface est érodée par places. Les annuli de croissance sont bien marqués et les plaques sont épaisses montrant qu'il s'agit d'un adulte. La nucale manque presque entièrement; la périphérique 2 gauche est pointue à l'extrémité externe du sillon des marginales 1 et 2 , mais l'encoche antérieure est large et faible. La forme est allongée avec un long plateau médian et des bosselures sous les costales à gauche. La bordure antérieure n'est pas relevée. La bordure postérieure est un peu disloquée à droite mais elle est quand même un peu redressée «en jupette" au niveau des périphériques 9 et 10, bien en place à gauche. Les sillons des écailles costales/ marginales-vertébrale 5 coïncident avec les sutures pleurales-suprapygale 2 /périphériques-pygale. Les neurales 2 à 7 sont différenciées régulièrement, alternativement octogonales et carrées; cela se voit grâce au bord gauche des neurales 2 et 3 préservées à leur contact avec les pleurales 2 et 3 gauches, alternativement courte et longue respectivement, et aux neurales 4 à 7 préservées comme les pleurales 4 à 6 , également alternativement courte, longue et courte. Après la neurale 7 carrée, la neurale 8 est hexagonale à côtés latéro-antérieurs presque aussi longs que les latéro-postérieurs. Les neurales ont donc successivement (de la 1 re à la 8 e ) 4-8-4-84-8-4-6 côtés. Les supaprapygales 1 et 2 sont des trapèzes séparés par une suture transversale un peu sinueuse, la première plus étroite et plus longue. La pygale est peu courbée ce qui semble indiquer une femelle, large à l'avant, à bords latéraux rectilignes divergents vers l'avant. La série vertébrale est relativement large (V3: largeur sur longueur : $155 \%$ ). Le pont s'étend de la périphérique 3 à la périphérique 7 , recevant les contreforts axillaire et inguinal respectivement. Le plastron est de type Testudo avec une charnière hypo-xiphiplastrale possible marquée par la rainure de gangue entre hypo-et xiphiplastrons mais l'hypoplastron participe au lobe postérieur. Le lobe antérieur est arrondi et étroit aux gulaires triangulaires qui sont à peine saillantes, courtes et qui, à l'arrière, parviennent à l'avant de l'entoplastron. Le bourrelet épiplastral dorsal est large et va jusqu'à l'entoplastron. Il est dorsalement un peu écrasé mais convexe (Fig. 7C2 en vue latérale). Il n'y a pas d'écailles axillaires et inguinales visibles. Les sillons huméro-pectoraux plutôt rectilignes passent juste au bord arrière de l'entoplastron et de ce fait les pectorales paraissent plus longues. Les sillons abdomino-fémoraux sont rapprochés de la suture hypo-xiphiplastrale constituant la charnière mais en restent écartés médialement et latéralement, avec une sinuosité entre les deux sur le côté. Les bords du lobe postérieur sont convergents vers les pointes xiphiplastrales, délimitant une encoche anale large et peu profonde, et ils sont un peu resserrés aux sillons fémoro-anaux. Les sillons fémoro-anaux sont parallèles aux bords de l'encoche anale, les anales n'étant pas beaucoup plus longues médialement que les fémorales et ne s'avançant pas vers la suture hypo-xiphiplastrale.

\section{COMPARAISON}

Par la charnière hypo-xiphiplastrale et l'ensemble de la morphologie il s'agit d'un membre de Testudo. À la différence des deux spécimens examinés précédemment, aucun caractère dérivé du groupe kenitrensis n'est présent. Presque tous les caractères, relativement primitifs, sont aussi présents chez Testudo graeca. Le seul caractère dérivé est la bordure postérieure un peu redressée et commune avec les espèces actuelles du genre. Toutefois deux points ne sont pas fréquents: 1) Les humérales touchent le bord postérieur de l'entoplastron. Or les Testudo actuels observés ont des humérales ne touchant pas le bord postérieur de l'entoplastron (sillon huméro-pectoral postérieur à l'entoplastron); toutefois, le sillon est très près de cette plaque chez certains actuels $T$. antakyensis et $T$. cyrenaica. D'autre part, ce sillon traverse sinueusement l'entoplastron chez un spécimen du Soltanien de Oualidia (Tableau 1) (Gmira 1995) attribué à T. graeca par son bourrelet épiplastral dorsal, mais s'en écartant du fait de ce sillon; et 2) Les abdominales ne joignent pas la charnière hypo-xiphiplastrale, ce qui est primitif pour Testudo et aussi présent dans le groupe kenitrensis sauf une exception et médialement seulement: comme dans ce groupe, l'hypoplastron participe davantage au lobe postérieur que chez les spécimens de T. graeca du Pléistocène supérieur du Maroc (Gmira 1995) et que chez les actuels, sauf chez des jeunes (jeune spécimen de Testudo graeca de Fez; MNHN.F, coll. REP 16). 


\section{DisCUSSION}

Présence de Testudo fossile au Maghreb

L'espèce actuelle Testudo graeca du Maghreb est proche de la forme primitive hypothétique d'où peuvent être issues les populations de Testudo du Plio-Pléistocène du Maroc, mais avec une bordure périphérique redressée et sans les apomorphies terminales du groupe kenitrensis (encoche antérieure trapézoïdale, vertébrale 1 éventuellement en lyre, série vertébrale plus étroite, absence de long plateau dorsal) et du clade kenitrensis (bourrelet épiplastral long et concave, anales longues médialement). Mais la forme primitive ancestrale n'y est pas vraiment connue. Avant le Pliocène d'Ahl al Oughlam, des restes rares de Testudininae incluant éventuellement Testudo ont été recensés au Maghreb (Lapparent de Broin 2000a) : "Testudo" semenensis Bergounioux, 1955 (holotype au MHNT) du début du Miocène supérieur (Jaeger 1977), du Djebel Sémène, Tunisie (Tableau 1), pourrait effectivement représenter le genre sous sa forme primitive. La surface est érodée et représentée par le moulage interne partiellement. La dossière est allongée avec un long plateau dorsal, la bordure antérieure est à peine encochée et non redressée; la bordure postérieure n'est pas étalée et redressée; les neurales sont régulièrement différenciées, la première et la troisième quadrangulaires et les neurales 6 à 8 hexagonales, avec les pleurales alternativement longues et courtes médialement; la division des deux suprapygales est transversale et la pygale est haute. Le plastron n'est conservé que par une partie du bourrelet épiplastral antérieur visible en coupe: il est recourbé, convexe et avec une poche gulaire; mais le reste n'est pas conservé de sorte qu'il n'est pas possible de voir la présence éventuelle d'une charnière hypo-xiphiplastrale qui identifierait le genre Testudo à coup sûr, notamment par rapport à Paleotestudo. Des restes de "Testudo" ont été signalés au Miocène supérieur (Vallésien) (Tableau 1) d'âge voisin de Bou Hanifia (Oued el Hammam) (Algérie) par Arambourg (1952), mais ils ne sont ni décrits ni figurés. D'autres restes de «Testudo" ont été signalés (Coppens 1971) à Hammada Damous, Tunisie, au Pliocène inférieur, c. 4,5 Ma, mais ni figurés ni décrits non plus. Enfin, des restes de plaques isolées d'un petit testudinidé terrestre pouvant représenter Testudo proviennent du Plio- cène inférieur d'Ichkeul, Tunisie (MNHN.F, dont 1949-1, 1950-1) et du Pléistocène d'Aïn Boucherit (Algérie) (MNHN.F, 1953-22-25), vers 2 Ma et donc plus récent qu'Ahl al Oughlam (Tableau 1) (Geraads 2010b). Par la suite au Pléistocène, Testudo est signalé en Algérie (notamment à Tighenif Algérie (MNHN.F, 1955-13 notamment) vers 700000 ans BP (Geraads 2010b) au Maroc et en Tunisie (Gmira 1995; Lapparent de Broin 2000a). Quand le matériel est déterminable spécifiquement et bien daté, c'est-à-dire au Pléistocène supérieur (Gmira 1995) et à l'Holocène, il s'agit de Testudo graeca avec cette exception pour la population du spécimen du Soltanien de Oualidia (Tableau 1) à sillon huméro-pectoral traversant l'entoplastron.

Par rapport à Paleotestudo (Miocène), l'espèce la plus primitive de Testudo se distinguerait par la présence de la charnière hypo-xiphiplastrale. Celle-ci est sûrement réalisée au Miocène supérieur, Vallésien supérieur (MN 10) de Grèce avec une suture proche du sillon abdomino-fémoral (Lapparent de Broin et al. 2006b, c ; Garcia et al. 2011) bien avant que cela se manifeste au Maroc au Plio-Pléistocène: l'on ne sait si une forme à charnière existait au Maghreb antérieurement, telle "T.» semenensis (début du Miocène supérieur). D'ailleurs, l'évolution du rapprochement du sillon abdomino-fémoral et de la suture hypo-xiphiplastrale a pu se faire éventuellement au Maroc parallèlement avec la Grèce, mais plus tardivement à partir d'une forme restée plus primitive.

Au Maroc, le spécimen de Testudo sp. A de la localité inconnue du cordon dunaire décrit ci-dessus pourrait représenter un premier stade d'évolution compatible avec la lignée de $T$. graeca au Maroc, qui serait issue directement de la forme primitive peut-être représentée par " $T$.» semenensis. Dans la partie supérieure du Pléistocène du Maroc (Tensiftien, Soltanien et Rharbien in Gmira [1995]), T. graeca semble bien représentée par le bord postérieur de la dossière redressé mais en outre par le sillon abdomino-fémoral qui s'unit davantage à la charnière, médialement et latéralement. Il est possible de considérer que, d'autre part, T. oughlamensis $\mathrm{n}$. sp. et T. kenitrensis se sont individualisées à partir du même stock primitif, formant la lignée kenitrensis qui pourrait n'être qu'un sous-groupe 

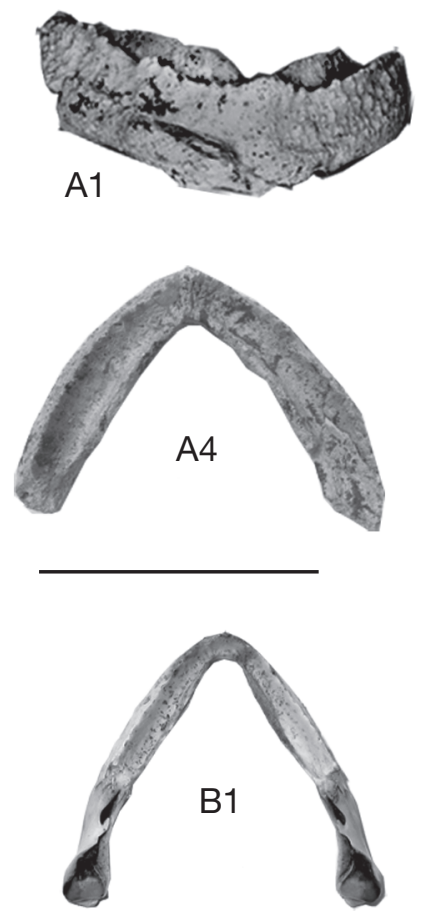

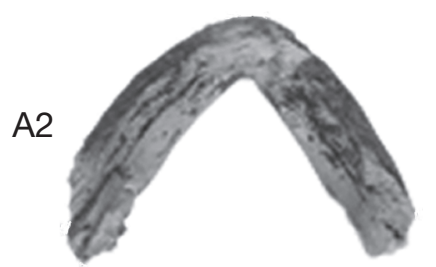

A3
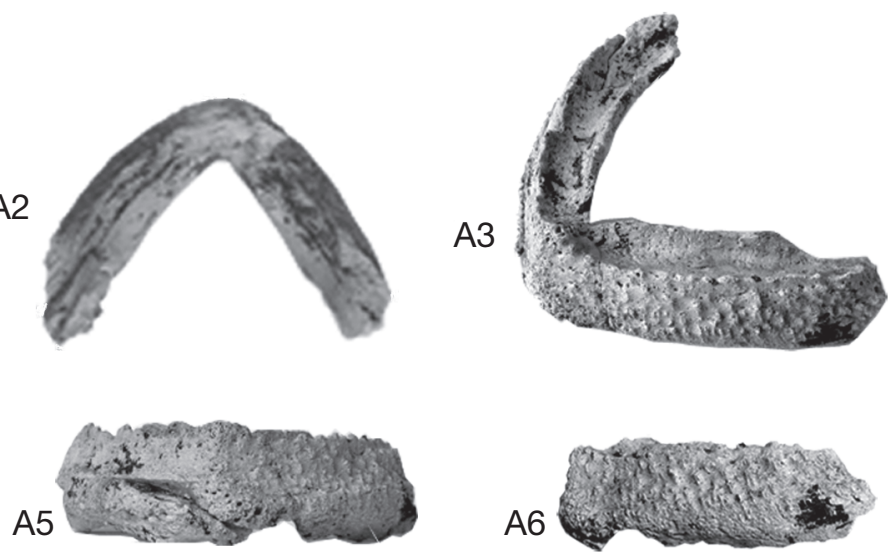

A6

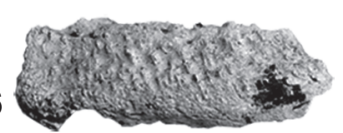

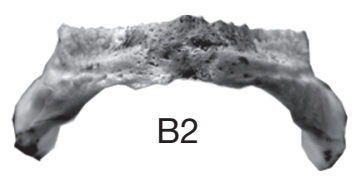

FIG. 8. - A, ?Centrochelys marocana n. sp., Ahl al Oughlam, Maroc, Pliocène supérieur, holotype, coll. INSAP. AaO-3017, dentaires fusionnés, A1-A6, vues dorso-antérieure, ventrale, dorso-latérale gauche, dorsale, frontale et latérale gauche; B, Centrochelys sulcata (Miller, 1779), Niger, MNHN.F, coll. REP 37 ; B1, B2, mandibule, faces dorsale et frontale. Échelles : A, $10 \mathrm{~cm}$; B, $5 \mathrm{~cm}$.

dans un groupe plus large de T. graeca. Les espèces Testudo sp. C de Dehar Mouak et Testudo sp. B d'Ar Rehamna pourraient représenter des premiers stades de cette lignée kenitrensis. La découverte de spécimens plus complets permettrait seule de vérifier ces hypothèses. Curieusement, la lignée kenitrensis s'éteint et disparaît au Pléistocène supérieur au profit de T. graeca.

Genre ?Centrochelys Gray, 1872

\section{?Centrochelys marocana $\mathrm{n} . \mathrm{sp}$.} (Figs 8 ; 9)

Geochelone sp. - Raynal et al. 1990: 316, 317. — Bailón 2000: 540, 542.

?Centrochelys sp. - Lapparent de Broin 2000a: 62; 2002: 130.
MATÉRIEL. - Holotype: individu représenté par une mandibule partielle (dentaires fusionnés), $\mathrm{AaO}-3017$, un humérus gauche partiel, $\mathrm{AaO}-3385$, un radius et un ulna gauches jointifs, $\mathrm{AaO}-180 \mathrm{a}$, b, le tibia droit, $\mathrm{AaO}-181$, le tibia gauche partiel, $\mathrm{AaO}-229$, un carpien radial gauche, sept phalanges unguéales, 33 ostéodermes.

DiAgNOSE. - Espèce géante de Testudininae se distinguant par la conjonction de sa carapace pouvant atteindre environ 180 à $200 \mathrm{~cm}$ pour une mandibule de $143 \mathrm{~mm}$ de large à l'arrière des dentaires et un tibia de $265 \mathrm{~mm}$ de long, ses dentaires à angle dentaire en $\mathrm{V}$ de 70 degrés, à bords externes arrondis avec un petit bec saillant et à surface très irrégulièrement tuberculée sous la rhamphothèque, à petite crête symphysaire et bord ventral symphysaire ne dépassant pas postérieurement le bord lingual dorsal, son tibia très robuste proximalement par rapport à distalement, ses radius et ulna à diaphyses relativement courtes entre leurs longs contacts proximal et distal, ménageant entre eux un espace large et court presque quadrangulaire, et son ulna arqué extérieurement. 
ÉTYMOLOGIE. - En référence au Maroc, pays de l'espèce.

LOCALITÉ ET HORIZON STRATIGRAPHIQUE TYPES. Remplissage continental dans la Carrière d'Ahl al Oughlam, située au sud-est de Casablanca, à gauche de la route S 106 allant de Casablanca à Tit Mellil. 33034'11"N, 07 30'44”W. Cordons dunaires de la côte de Casablanca à Kenitra, Maroc. Unité morpho-sédimentaire de Sidi Messaoud. Carte géologique, feuille de Casablanca-Mohammedia (Destombes 1987). Pliocène supérieur, âge estimé à 2,5 Ma (Raynal et al. 1990; Geraads et al. 2010).

\section{DESCRIPTION}

Les restes de la tortue étaient associés dans le remplissage d'une petite grotte; il n'y a pas d'os d'un seul côté en double, les deux tibias correspondent exactement à un droit et un gauche d'un même individu et les dimensions relatives des os des membres correspondent à celles d'un seul individu par comparaison avec Centrochelys sulcata (Miller, 1779) et Stigmochelys pardalis (Bell, 1828) actuels.

\section{Mandibule, AaO-3017 (Fig. 8A, Tableau 2)}

Les branches dentaires sont fusionnées suivant un $\mathrm{V}$ à angle de $70^{\circ}$, la surface de leurs bords externes un peu arrondie mais l'avant est pointu. Les bords dorsal et ventral de chaque dentaire sont parallèles en vues frontale et latérale (Fig. 8A5, A6) ; les surfaces triturantes sont concaves avec les bords lingual et labial à peine divergents vers l'arrière, la concavité longitudinale entre eux étant dilatée médialement, le bord labial bien dentelé et épais, le lingual peu dentelé et plus mince, et avec une légère crête dorsale sagittale symphysaire; le bord labial forme un petit bec pointu à l'avant, saillant médialement, et le bord lingual s'atténue à la symphyse formant une petite encoche verticale symphysaire (Fig. 8A1). Sur la face externe du dentaire, la partie décorée marque la place de la rhamphothèque; elle s'étend tout autour sur presque toute la hauteur dentaire se raccourcissant un peu vers l'arrière; elle est crênelée, tuberculée et saillante au dessus et devient plus lisse en dessous et au milieu; les tubercules s'alignent ou alternent, de la rangée supérieure sous le bord labial dentelé jusqu'à la rangée inférieure, limite inférieure de la rhamphothèque. Les dentaires sont un peu en retrait à la base (inclination vers l'intérieur visible en vue latérale), sous la marque du bord inférieur de la rhamphothèque (Fig. 8A6) et le bord ventral symphysaire ne dépasse pas postérieurement le bord dorsal symphysaire de la surface triturante (visible en vue dorsale; Fig. 8A4).

\section{Humérus. AaO-3385 (Fig. 9A1-A5; Tableau 2)}

L'humérus gauche est conservé dans sa partie diaphysaire avec proximalement la base des trochanters et de la tête et, distalement, l'élargissement vers la facette trochléaire. Il est robuste, progressivement et modérément aminci médialement. La diaphyse est modérément courbée (angle d'environ 150-160 en position latérale (Fig. 9A1, A2) et renflée sur ses $2 / 3$ sous la tête (Fig. 9A3) s'étrécissant médialenent pour s'élargir vers la facette distale (Fig. 9A4); la tête se courbe dorsalement sur la diaphyse sur un angle très ouvert (environ 135 $5^{\circ}$; l'angle d'ouverture des deux trochanters est d'environ $20^{\circ}$ et la divergence distale s'amorce, plus large que la divergence des trochanters (Fig. 9A4) : la facette distale était large par rapport à la diaphyse à sa mi-hauteur.

\section{Radius et Ulna, AaO-180a, $b$}

(Fig. 9B1-B4; Tableau 2)

Les radius et ulna gauches sont associés. Le radius est long proximalement dans son contact ave l'ulna et très étalé distalement avec une facette distale ovoïde ventralement. La tête de l'ulna est courte et large. Son bord externe fortement arqué forme une crête dans sa partie supérieure sous la tête. Le radius et l'ulna sont unis proximalement et distalement sur une grande longueur, isolant entre eux une fenêtre ovale presque quadrangulaire relativement courte. Ils sont tous deux étroits en section, amincie.

\section{Carpien radial gauche (Fig. 9E1-E3; Tableau 2)}

Large pour sa hauteur, sa facette proximale double recevait le radius et le carpien central-radial et sa facette distale montre trois facettes d'articulation avec les trois premiers métacarpiens; médialement, il s'unissait au carpien central adjacent.

\section{Tibias, Tibia droit, AaO-181 (Fig. 9C1-C4;}

Tableau 2); Tibia gauche, AaO-529 (Fig. 9D1, D2) Le tibia droit est complet, le gauche est conservé aux deux tiers inférieurs. Sur le tibia droit, la fa- 
TABLEAU 2. - Mesures d'éléments de ?Centrochelys sp., Ahl al Oughlam, Pliocène, Maroc. Abréviations : ant, antérieure ; H, hauteur ; L, longueur ; I, largeur ; méd, médiane ; post, postérieure ; sup, supérieure.

\begin{tabular}{llccc}
\hline Cf. ?Centrochelys marocana $\mathbf{n}$. sp. & & Dimensions $\mathbf{( m m})$ & Figures \\
\hline Mandibule (AaO-3017) & Dentaires fusionnés & L méd & 99 & Fig. 8A1-A6 \\
& & I post & 143 & Fig. 8A5 \\
& & H ant & 40,7 & Fig. 8A4 \\
& Symphyse & L sup & 20,9 & Fig. 8A3, A4 \\
& Surface triturante & I post & 23,1 & Fig. 9A1-A5 \\
& Préservée & $\mathrm{L}$ & 310 & \\
\hline Humérus gauche (AaO-3385) & Estimée & $\mathrm{L} \times \mathrm{I}$ & $265 \times 140$ & Fig. 9B1-B4 \\
& & $\mathrm{L} \times \mathrm{I}$ & $79 \times 98$ & Fig. 9C1-C4 \\
\hline Tibia droit (AaO-181) & Facette distale & $\mathrm{L}$ & 275 & Fig. 9B2 \\
\hline Radius (AaO-180a) & & $\mathrm{L}$ & 263 & Fig. 9B2-B4 \\
\hline Ulna (AaO-180b) & & $\mathrm{L}$ & 98 & Fig. 9B2 \\
\hline Radius-ulna fenestra & & $\mathrm{L}$ & 47,1 & Fig. 9E1-E3 \\
\hline Carpien radial gauche & Facette proximale & $\mathrm{L}$ & 150,7 & \\
\hline Phalanges unguéales & AaO-182b & $\mathrm{L}$ & c. 68 & Fig. 9F6 \\
\hline Ostéoderme & AaO-182a & $\mathrm{L} \times \mathrm{I}$ & c. $97 \times 57$ & Fig. 9G3 \\
\hline
\end{tabular}

cette proximale, en relation avec la facette distale du fémur, est très large (environ $53 \%$ de la longueur maximale de l'os) par rapport à la facette distale. La facette proximale est un peu érodée à gauche et fait complètement défaut sur le tibia droit; elle est grande, grossièrement trapézoïdale avec des côtés arrondis et, au dessous, la partie tibiale proximale est corrélativement très robuste. La diaphyse est bien incurvée médialement et concave sur trois de ses faces sauf sur la face antérieure; à partir de la mi-longueur de cette face se trouve une large crête d'insertion musculaire saillante, laquelle s'étend distalement jusqu'à la base de l'os. La diaphyse porte en outre une crête longitudinale oblique sur les deux tiers inférieurs de sa face postérieure. La facette distale des deux tibias est concave, polygonale avec un fort décrochement latéral, s'incurvant autour du gros tarsien central.

\section{Phalanges unguéales (Fig. 9F1-F7; Tableau 2)}

Sur les sept conservées, une est plus courte et triangulaire, de l'extrémité latérale externe d'un pied peut-être. Les autres sont plus allongées et larges à leur extrémité distale, laquelle est frangée (bord sinueux), provenant aussi bien du pied que de la main.

\section{Ostéodermes (Fig. 9G1-G11; Tableau 2)}

Trente trois ostéodermes sont conservés. Ils recouvraient les membres antérieurs et postérieurs sans qu'une distinction soit effectuable. Ils varient d'arrondis et plats à plus ou moins allongés et pointus.

\section{COMPARAISON}

Les comparaisons portent principalement sur les restes très partiels et rares de grosses tortues terrestres fossiles du Néogène de la partie Nord d'Afrique et leur parent potentiel actuel, Centrochelys sulcata (répartition en Afrique, d'Est en Ouest au Sahel-subSahel; Roset et al. 1990 ; Iverson 1992) (MNHN.F, coll. REP et MNHN.ZA), sur l'autre grand testudininé actuel d'Afrique, Stigmochelys pardalis (partie orientale d'Afrique: Iverson 1992) (MNHN.ZA et RA) et sur la grande européenne "Cheirogaster" perpiniana (Depéret, 1885) (MNHN.F, 1887-26) (Bourgat \& Bour 1983; Depéret \& Donnezan 1890-1897) du Pliocène du Roussillon (MN 15) (Tableau 1). Toutes ces tortues ont en commun d'être de grande taille, de ne pas avoir d'écaille cervicale sur la dossière (quand elle est connue) ni dorsale ni ventrale et d'avoir un morphotype pygale-suprapygales particulier: suprapygale 2 lenticulaire embrassée par la surapygale 1 et la pygale, 
et traversée par le sillon entre l'écaille vertébrale 5 et l'unique écaille supracaudale; ce morphotype "Geochelone» représente un grade qui fut attribué à ce genre quoique existant en fait dans plusieurs lignées et rarement chez l'espèce type Geochelone elegans (Schoepff, 1795) (Lapparent de Broin 2002, 2003, 2008). Les restes de tortues fossiles géantes d'Europe (Lapparent de Broin 2002) sont la plupart mal connues et provisoirement attribuées au genre Cheirogaster Bergounioux, 1935; celle de Perpignan est la seule complète. Il conviendrait de séparer génériquement l'espèce type $C h$. maurini Bergounioux, 1935 de l'Éocène supérieur de France (voir Bergounioux 1935; Broin 1977) et les restes provenant du Néogène européen (voir Lapparent de Broin 2002); leur révision n'est pas l'objet de ce travail, mais les guillemets au genre attirent l'attention sur le problème. Quelques comparaisons sont aussi faites avec les grandes formes de Madagascar (à écaille cervicale présente au moins ventralement), Aldabrachelys grandidieri (Vaillant, 1885 ) et $A$. abrupta (Grandidier, 1868) originaires d'Afrique (Bour 1994).

\section{Mandibule}

Les dentaires fusionnés d'Ahl al Oughlam sont comparables à ceux de $C$. sulcata et à ceux de toutes les tortues terrestres adultes. Ce sont des mandibules de tortues végétariennes avec une longue concavité supérieure triturante sur chaque branche dentaire entre les bords labial et lingual; mais chacune peut présenter des particularismes. Celle de ?C. marocana n. sp. d'Ahl al Oughlam ressemble à celle de $C$. sulcata (Fig. 8B) par sa symphyse dont le bord ventral ne dépasse pas le bord dorsal postérieurement et par le petit bec antérieur bien délimité médialement sur le bord labial. ?C. marocana n. sp. s'en distingue par l'arrondi externe des dentaires, par la légère crête dorsale sagittale, les bords lingual et labial plus épais et moins coupants et l'ornementation du pourtour externe tuberculée alternée (plus crênelée chez sulcata). Elles se distinguent aussi l'une de l'autre par l'angle entre les branches dentaires plus ouvert à Ahl al Oughlam, de $70^{\circ}$, pour c. 55 à $65^{\circ}$ chez C. sulcata. La mandibule de Stigmochelys a un bord externe un peu arrondi s'élevant vers l'avant pour former un petit bec, mais non délimité et elle a un angle dentaire plus ouvert $\left(65-67^{\circ}\right)$ se rapprochant de celui de? C. marocana n. sp. Elle se distingue des précédentes par le bord ventral symphysaire plus en retrait antérieurement et dépassant le bord dorsal postérieurement (visible en vue dorsale). Une partie des différences entre $C$. sulcata et l'espèce d'Ahl al Oughlam peut tenir à la taille: la dilatation de la concavité triturante plus marquée chez ?C. marocana n. sp. (concavité qui s'étrécit davantage vers l'avant chez $C$. sulcata) et ses bords plus arrondis que chez C. sulcata, par exemple. Mais non en ce qui concerne l'angle dentaire: il est voisin de $60^{\circ}$ chez la géante "Ch." perpiniana (mandibule de $11 \mathrm{~cm}$ de large à l'arrière des dentaires pour une carapace de $112 \mathrm{~cm}$ de long) montrant que la valeur de l'angle ne tient pas à la taille. Chez celle-ci, il y a aussi un petit bec antérieur, le bord postérieur symphysaire ventral ne dépasse pas le bord dorsal mais les dentaires sont peu ornés et en dessous, leur face lisse est fortement inclinée vers l'intérieur. La mandibule de "Cheirogaster» sp. du Miocène supérieur (Vallésien inférieur, MN 9) de Catalogne (Lujan et al. 2010) présente aussi un angle inférieur à $60^{\circ}$ et elle paraît peu ou pas ornementée comme "Ch." perpiniana. Ces mandibules attribuées à Cheirogaster sont relativement plus étroites. L'angle varie de $c .65^{\circ}$ à $c .70^{\circ}$ chez Aldabrachelys grandidieri du Pléistocène de Madagascar en fonction de la taille (longueur de la mandibule complète de 101 à $130 \mathrm{~mm}$ ) (Bour 1994), atteignant donc la valeur de la grande mandibule d'Ahl al Oughlam. Mais la morphologie des dentaires est différente: notamment, la décoration est faible, l'extrémité antérieure est arrondie, la symphyse est plus courte sans crête sagittale et il y a une crête intermédiaire

FIG. 9. - ?Centrochelys marocana n. sp., Ahl al Oughlam, Maroc, Pliocène supérieur, holotype, coll. INSAP: A1-A5, AaO-3385, humérus gauche partiel, vues médio-ventrale, externe, dorsale, ventrale et médiale ; B, AaO-180 ; B1-B4, radius et ulna gauches ; B1, AaO-180b, radius, vue médiale ; B2, AaO-180a, b, radius et ulna associés, vue dorsale ; B3, B4, AaO-180a, ulna, vues médiale et distalo-médiale ; C, AaO-181 ; C1-C4, tibia droit, vues postérieure, proximale, antérieure et médiale ; D, AaO-529 ; D1, D2, tibia gauche, vues postérieure et distale ; E, AaO-4302 ; E1-E3, carpien radial gauche, vues dorsale, proximale et distale ; F1-F7, phalanges unguéales ; F1, AaO-ssn, F2, AaO-ssn ; F3, AaO-4323, F4, AaO-3392 ; F5, AaO-2868 ; F6, AaO-182 ; G1-G11, ostéodermes ; 

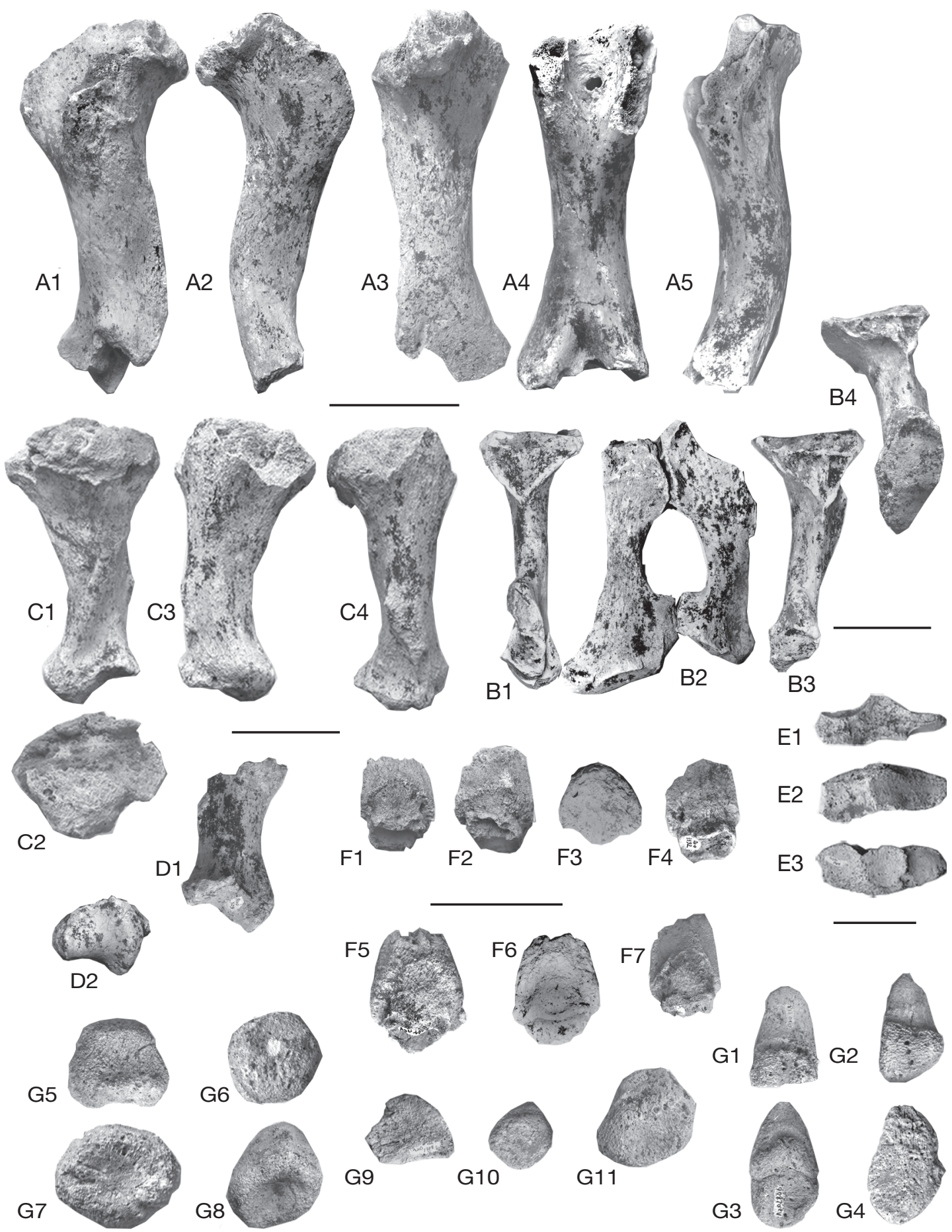

G1, AaO-1784 ; G2, AaO-2869 ; G3, AaO-2886 ; G4, AaO-2213 ; G5, AaO-2211 ; G6, AaO-2864 ; G7, AaO-3444 ; G8, AaO-2867 ; G9, AaO-ssn ; G10, AaO-3444 ; G11, AaO-4297. Échelles : 10 cm. 
entre les bords lingual et labial. La mandibule d'Ahl al Oughlam est donc différente de celle des taxons auxquels elle est comparée ici par la conjonction de ses caractères, tout en ressemblant davantage à celle de Centrochelys sulcata par son bec mieux délimité, ses bords symphysaires et l'ornementation marquée.

\section{Humérus}

Il a une diaphyse relativement plus robuste que chez $C$. sulcata, plus large médialement et plus renflée sous les trochanters, et la tête se courbe dorsalement sur la symphyse sur un angle un peu plus ouvert; mais la faible courbure de la diaphyse (150-160 chez sulcata), l'angle d'ouverture des deux trochanters et la divergence distale vers les deux parties de la facette distale (large par rapport à la diaphyse) sont identiques. Un humérus partiel de ?Centrochelys punica (Arambourg, 1979) (in Lapparent de Broin 2000a) du Pliocène du lac Ichkeul, Tunisie (c. 3,5 Ma) (MNHN.F 1950-1-41) n'a pas été figuré, pas plus qu'une portion de bordure périphérique; sa longueur préservée est de $352 \mathrm{~mm}$ et il pouvait atteindre $450 \mathrm{~mm}$, évoquant une taille de carapace plus grande que celle estimée auparavant d'après l'holotype (un fémur) (Lapparent de Broin 2002); cet humérus est très proche de celui d'Ahl al Oughlam, quoique pouvant même être un peu plus grand; un peu moins complet à la tête il est pareillement conservé du côté distal: la diaphyse est aussi robuste et renflée médialement, l'angle des trochanters est voisin (environ $25^{\circ}$ ), la courbure diaphysaire (mesurée en vue latérale) est voisine (angle d'environ $150^{\circ}$ ); la divergence distale est à peine inférieure et la divergence des trochanters à peine supérieure. Cet humérus est conféré à ?Centrochelys comme celui d'Ahl al Oughlam. Par ailleurs un humérus provenant du Djebel Krechem El Artsouma, Tunisie centrale, Miocène supérieur, Vallésien supérieur (Tableau 1), fut attribué à Geochelone sp. (in Geraads 1989) puis à ?Centrochelys sp. (in Lapparent de Broin 2000a). Il est long de $237 \mathrm{~mm}$ de long, un peu plus petit que celui d'Ahl al Oughlam et que celui d'Ichkeul. Sa face ventrale montre la même divergence des trochanters et, à l'épiphyse distale, sa face latérale montre exactement la même courbure de diaphyse et la même courbure de la tête sur la diaphyse que l'humérus d'Ahl al Oughlam: comme les autres restes géants d'Afrique, il est aussi attribué à cf. ?Centrochelys sp.

Chez "Ch.» perpiniana (humérus de $302 \mathrm{~mm}$ de long) la divergence distale est semblable à celle de ?C. marocana n. sp. mais la diaphyse est un peu plus courbée $\left(c .130-140^{\circ}\right)$, la courbure de la tête un peu plus accentuée mais, surtout, l'angle des trochanters est moins ouvert (environ $16^{\circ}$ ). Un grand spécimen du Miocène supérieur de France, du Bois de Fabrègues (Var) (MNHN.F.APF1), indéterminé mais situé dans la zone paléogéographique de "Cheirogaster» leberonensis (Depéret, 1890), aussi du Miocène supérieur (Lapparent de Broin 2002), a une courbure d'humérus voisine, de l'ordre de $140^{\circ}$. Un humérus partiel sans la partie distale (MNHN.F.SLQ996) (315 mm préservés) du Miocène supérieur de Karabourun, près de Thessalonique, Grèce, qui pourrait aussi être attribué à Cheirogaster du fait de sa localisation européenne ("Testudo»sp. in Arambourg \& Piveteau 1929), ressemble à celui d'Ahl al Oughlam par sa courbure et l'écartement de ses trochanters, mais sa tête est plus courbée sur la diaphyse comme chez «Ch.» perpiniana. Stigmochelys pardalis, l'autre grande tortue terrestre africaine actuelle est, comme C. sulcata, petite par rapport à la forme d'Ahl al Oughlam (carapace d'environ $85 \mathrm{~cm}$ ). Chez $S$. pardalis, la courbure de l'humérus, de l'ordre de $140^{\circ}$, est plus forte que chez C. sulcata et ?C. marocana n. sp., et la facette distale est plus recourbée médialement; elle est aussi relativement moins large par rapport à la diaphyse médialement. L'angle des trochanters est en $\mathrm{U}$ au lieu d'en $\mathrm{V}$ large chez $C$. sulcata et chez ?C. marocana n. sp., espèces où les trochanters sont plus écartés proximalement; chez $S$. pardalis l'angle est nettement plus fermé dans la partie proximale où les deux trochanters sont inclinés l'un vers l'autre (pouvant passer de $20^{\circ}$ à leur union à $10^{\circ}$ proximalement). Chez Aldabrachelys abrupta et A. grandidieri, la courbure semble variable de 130 à $140^{\circ}$ mais, surtout, le point de courbure est déplacé vers la facette distale; l'humérus est très robuste, très large à ses extrémités; la diaphyse est immédiatement plus divergente proximalement et dorsalement après le point de rétrécissement médial; corrélativement, les trochanters sont très écartés à la base (angle de $60^{\circ}$ ) mais ils se rapprochent proximalement un peu chez A. abrupta. 
Une plus forte courbure diaphysaire de l'humérus est fréquente chez les Testudininae terrestres dont la carapace est bombée comme Stigmochelys et Testudo graeca. Un angle entre les trochanters se refermant proximalement est également visible chez d'autres genres de Testudininae terrestres bombés tels les petits Testudininae paléarctiques, dont Testudo, et tels Astrochelys radiata de Madagascar et le grand Namibchersus namaquensis de Namibie. Chez ces derniers et à la différence d'Aldabrachelys, le rapprochement proximal des trochanters va de pair avec la facette distale plus étroite et la courbure plus forte. Chez C. sulcata, d'un humérus de $84 \mathrm{~mm}$ à un humérus de $105 \mathrm{~mm}$ de long (mesuré médialement), la courbure de la diaphyse diminue un peu mais elle reste toujours nettement plus faible que chez Stigmochelys et Namibchersus, formes à carapace bombée à la différence de Centrochelys et de "Ch."perpiniana. En fin de compte, les humérus africains les mieux conservés attribués à ?Centrochelys se ressemblent par la conjonction de la plus faible courbure diaphysaire, l'écartement des trochanters et la relative largeur de la facette distale mais ils peuvent être peu distincts de ceux, européens, que l'on attribue à "Cheirogaster": en fait, la courbure des humérus est ou non variable d'une espèce à l'autre chez Centrochelys et "Cheirogaster", mais elle reste modérée, la courbure étant bien dépassée chez Stigmochelys. Les fémurs aussi sont plus rectilignes comme les humérus dans les formes moins bombées comme Centrochelys et "Cheirogaster" par rapport à Stigmochelys ou Testudo: ceux préservés d'Afrique, du Miocène inférieur (Burdigalien) du Djebel Zelten, Libye (Tableau 1) (MNHN.F Z 1961, QB 55) comme celui de ?C. punica du Pliocène d'Ichkeul (holotype MNHN.F 1948-2-39), et comme ceux de C. sulcata (Lapparent de Broin 2002: fig. 16) sont semblables par leur diaphyse rectiligne; alors que chez "Ch." perpiniana, la diaphyse du fémur est rectiligne dans sa grande partie supérieure mais un peu incurvée vers la facette distale; chez "cf. Ch." schafferi (Szalai 1931; Lapparent de Broin 2002) de Samos, Miocène, la diaphyse est rectiligne, à peine légèrement incurvée, plus semblable à Centrochelys. Il est donc assez difficile de distinguer Centrochelys et toutes les espèces attribuées à "Cheirogaster" par les fémurs et les humérus alors que les plastrons, hélas pas toujours connus, se distinguent aisément (voir ci-dessous). En attendant une meilleure définition de ces "Cheirogaster", les fémurs de ces espèces africaines fossiles plus conformes à l'actuelle C. sulcata sont attribués à cf. Centrochelys; comme leurs humérus sont semblables à celui de l'espèce d'Ahl al Oughlam, cette dernière est aussi rapportée au genre, ce qui n'est pas contredit par les autres éléments, mandibule, et os des membres, malgré leurs spécificités. Il en est de même pour l'espèce indéterminée du Miocène du Djebel Krechem El Artsouma. Les carapaces de ?C. punica et de l'espèce d'Ahl al Oughlam ?C. marocana n. sp. ne sont que peu (reste non significatif à Ichkeul) ou pas (à Ahl al Oughlam) préservées, mais étant donné la faible courbure de leurs os longs, elles peuvent être supposées relativement basses comme chez C. sulcata et comme l'est celle d'un spécimen de Centrochelys sp. provenant du Mio-Pliocène inférieur du Maroc à Lissasfa, étudié ci-dessous. Il en est de même pour "Cheirogaster" perpiniana.

\section{Radius et ulna}

Par rapport à $C$. sulcata, les os d'Ahl al Oughlam sont plus étendus proximalement et la fenêtre entre les deux os en vue latérale est plus courte et plus large, étant presque quadrangulaire. Mais la forme distale et l'aplatissement des deux os sont très comparables. Chez "Ch. perpiniana» les deux os sont plus cylindriques, moins étalés distalement et la fenêtre entre eux est longue et étroite. Chez l'espèce indéterminée de Karabourun, les os (MNHN.F.SLQ998, SLQ999) sont aussi moins cylindriques que chez perpiniana, comme à Ahl al Oughlam et chez sulcata, la fenêtre est également un peu élargie comme à Ahl al Oughlam mais elle reste plus longue; les extrémités sont étalées comme à $\mathrm{Ahl}$ al Oughlam mais un peu plus longues. Il en est de même pour les radius et ulna de "Ch.» cf. schafferi de Pikermi (Bachmayer 1967; Lapparent de Broin 2002): Ainsi, dans tous les cas la fenêtre est plus longue et plus ovale que chez ?C. marocana n. sp., unique ici dans sa morphologie. 


\section{Tibia}

Sa courbure est identique à celle de $C$. sulcata mais chez celle-ci, un tibia de $55 \mathrm{~mm}$ de long (carapace d'environ $34 \mathrm{~cm}$ alors que la longueur maximum connue est d'environ $90 \mathrm{~cm}$ ) est plus gracile: relativement plus étroit pour sa longueur et avec une crête inférieure moins marquée mais identique dans sa proportion sur l'os et le degré de saillie; la crête postérieure est peu marquée et la facette proximale est quadrangulaire. Chez $S$. pardalis, la courbure du tibia est moins accentuée que chez les deux espèces précédentes; les crêtes sont aussi moins accentuées que chez ?C. marocana n. sp., mais la crête oblique postérieure est un peu plus marquée que chez sulcata rappelant ?C. marocana n. sp. Chez «Ch.» perpiniana, le tibia est relativement aussi robuste qu'à $\mathrm{Ahl}$ al Oughlam mais il est plus élargi distalement et moins proximalement. Un tibia partiel préservé d'une tortue géante du Pliocène de Lesbos (Grèce) et attribué à cf. "Cheirogaster» aff. schafferi est en revanche relativement plus gracile et plus incurvé au dessus et en dessous de la crête musculaire, laquelle est plus haute et plus saillante de profil (Lapparent de Broin 2002). D'après l'observation de squelettes de diverses tortues terrestres en collection au Muséum national d'Histoire naturelle (F, RA, ZA), il y a une variabilité dans la gracilité, la saillie des crêtes et la courbure avec les espèces sans que des caractères qui pourraient avoir une application générique ne se distinguent. Tout au moins les tibias d'Ahl al Oughlam se distinguent de ceux de C. sulcata par leur robustesse: cette différence n'est pas étonnante étant donné la différence de taille considérable.

\section{Phalanges unguéales (Fig. 9E1-E7)}

À notre connaissance, ce sont les premières griffes de tortues géantes fossiles identifiées dans la partie Nord de l'Afrique incluant l'aire de répartition de Centrochelys, d'est en ouest (Roset et al. 1990) : elles ne se distinguent pas de celles trouvées en Europe, par exemple chez cf. Cheirogaster aff. schafferi du Pliocène de Lesbos (Lapparent de Broin 2002, Figs 4-6) ni de celles de "Ch.» cf. schafferi du Miocène de Pikermi (Bachmayer 1967). Taille mise à part, elles ne sont pas bien différentes de celles d'espèces d'autres genres actuels variés à écaille cervicale présente, sans relation avec Centrochelys, telles Astrochelys radiata de Madagascar (nettement plus petite) et la grande Aldabrachelys gigantea d'Aldabra, par exemple.

\section{Ostéodermes (Fig. 9G1-G11)}

De même que pour les phalanges, ce sont les premiers récoltés fossiles pour les formes géantes du Nord de l'Afrique. Ils sont comparables à ceux des genres actuels continentaux non insulaires (chez ceux-ci, l'armure osseuse dermique des membres disparaît) mais ils diffèrent par leurs grandes taille et épaisseur. Ils ressemblent à ceux des tortues géantes attribuées à "Cheirogaster», en Grèce (Samos, Pikermi, Lesbos) comme en France (Perpignan). Comme chez les actuels et "Ch.» perpiniana, il y a des ostéodermes ronds alternant avec des plus longs et pointus au-dessus des membres y compris les cruraux (de la cuisse) plus coniques. Chez les actuels, leurs forme et arrangement permettent des distinctions spécifiques, exclues ici avec des os isolés.

\section{Dimensions (TABleaU 2)}

Par comparaison avec les espèces actuelles Stigmochelys pardalis et Centrochelys sulcata, le rapport de longueur du tibia par rapport à l'humérus, voisin de $62,25^{\circ}$, et le rapport de longueur de l'ulna par rapport à l'humérus, de $62,2^{\circ}$, permettent d'envisager une longueur d'environ $42 \mathrm{~cm}$ environ pour l'humérus du Maroc. La longueur de la carapace peut varier de 180 et $200 \mathrm{~cm}$, avec une marge d'incertitude importante car le rappport des os des membres et surtout de la mandibule à la longueur de la carapace n'est pas constant. Cela en fait une des plus grandes tortues terrestres connues d'Afrique, la plus grande avec ?C. punica de Tunisie. En Europe, les espèces rapprochées de «Ch.» schafferi de Lesbos et Samos (Grèce) peuvent avoir des dimensions voisines, de même que Megalochelys sivalensis Falconer \& Cautley, 1837 en Inde (Falconer \& Cautley 1837; Lapparent de Broin 2002: tableau 2). L'espèce ?C. punica, définie sur un fémur et auquel un humérus partiel et un fragment de carapace sont référés, ne peut pas être vraiment comparée à ?C. marocana n. sp. car ces deux os ne paraissent pas diagnostiques au niveau spécifique. Mais une synonymie pourrait être envisagée avec davantage de matériel, dont nécessairement les dentaires et l'association radius-ulna. 


\section{Centrochelys sp.}

(Fig. 10)

MATÉRIEL. - Coll. INSAP, LSF-5, moule interne de la dossière avec plastron; un œuf, coll. INSAP, LSF-6

LOCALITÉ ET HORIZON STRATIGRAPHIQUE. - Lissasfa, Casablanca, Maroc, grand cordon dunaire de Marchich qui, à $244 \mathrm{~m}$, domine la plaine de Médiouna (Raynal et al., 1999). Unité morpho-sédimentaire de Médiouna/Bouskoura, Miocène supérieur/Pliocène inférieur, c. 5.5 Ma (Tableau 1) (Raynal et al. 1999; Geraads et al. 2010).

\section{DESCRIPTION}

\section{Carapace}

Le spécimen est représenté par le moule interne de la dossière avec le relief interne des plaques pleurales et des périphériques, lesquelles sont très hautes. La carapace est relativement basse (elle a pu être un peu aplatie post-mortem). Le plastron est bien conservé, visible ventralement. Il est plat et à très faible saillie gulaire, un peu érodée: il peut appartenir à une femelle; les sutures ouvertes indiquent un spécimen jeune. Le lobe antérieur est à peine plus étroit et plus court que le lobe postérieur (mesurés au fond des encoches axillaire et inguinale respectivement) et le pont est plus long. Les bords latéraux du lobe antérieur sont régulièrement arrondis jusqu'aux gulaires qui forment un bord antérieur assez transversal, un peu érodé mais peu saillant. Le bourrelet épiplastral dorsal n'est pas dégagé de la gangue. L'entoplastron est très grand, aussi long que large et prolongé postérieurement au lobe antérieur médialement à l'avant du pont. Le lobe postérieur a des bords latéraux fort convergents vers les pointes xiphiplastrales, lesquelles sont assez pointues du fait de l'étroitesse et de la profondeur relatives de l'encoche anale; le bord latéral est arrondi jusqu'aux anales; il y a un resserrement au sillon fémoro-anal puis le bord latéral est rectiligne comme les bords de l'encoche anale. Les hypoplastrons forment une bonne part du lobe postérieur (presque un tiers latéralement).

\section{Dimensions}

Longueur maximum du plastron $355 \mathrm{~mm}$; largeur au sillon abdomino-fémoral : $174 \mathrm{~mm}$.
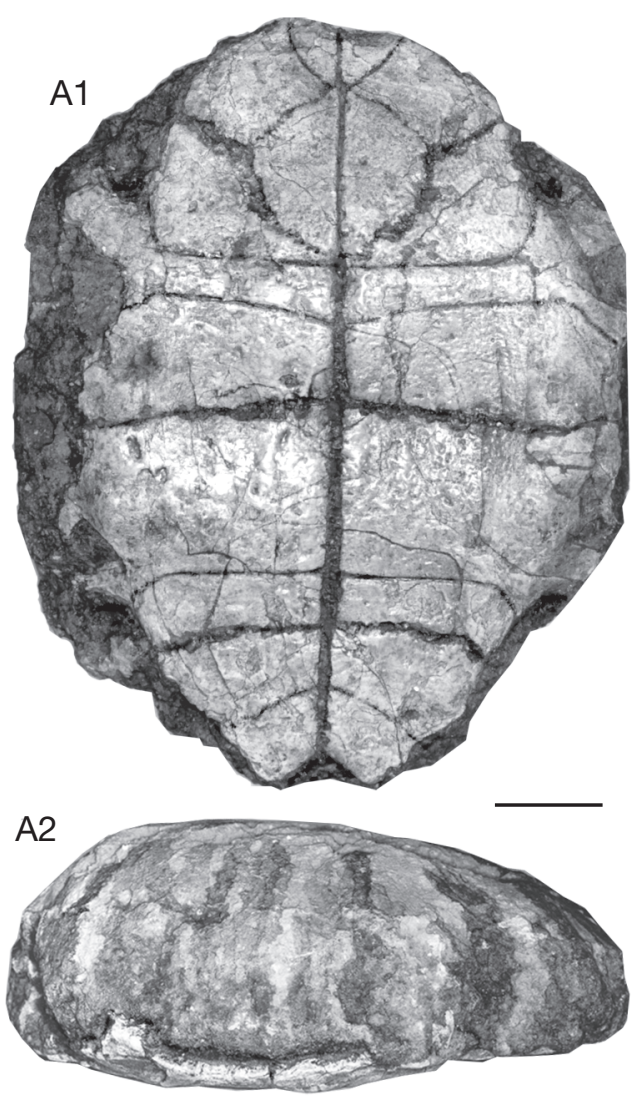

FIG. 10. - Centrochelys sp., Lissasfa, Maroc, Miocène supérieur/ Pliocène inférieur, coll. INSAP, LSF-5 : A1, Plastron, face ventrale; A2, carapace (avec moule interne de la dossière) face latérale gauche. Échelle : $5 \mathrm{~cm}$.

\section{Écailles}

Les gulaires, en triangle ventral, ont un bord latéral arrondi et se prolongent jusqu'à l'entoplastron qu'elles recouvrent à peine. Les humérales sont longues, le sillon huméro-pectoral étant postérieur au bord postérieur du grand entoplastron mais proche. Les sillons huméro-pectoral et abdomino-fémoral sont largement rectilignes avant un court recourbement latéral vers les encoches axillaire et inguinale respectivement. Les pectorales sont courtes: le sillon pectoro-abdominal est proche de l'huméro-pectoral, sinueux, un peu infléchi vers l'arrière médialement et (davantage) latéralement. La partie rectiligne du sillon abdomino-fémoral est un peu avancée par rapport au fond des encoches inguinales. Les anales 
couvrent la moitié postérieure des xiphiplastrons et le sillon fémoro-anal est oblique, rectiligne et parallèle au bord de l'encoche anale. Les abdominales sont les plus longues médialement, suivies des humérales, des fémorales, des anales, des pectorales et des gulaires.

\section{Euf}

Dimensions: $66 \times 58 \times 50 \mathrm{~mm}$. Arrondi mais un peu aplati post-mortem et la coquille érodée par places, il appartenait à une tortue de grande taille. La forme arrondie se trouve chez les Cheloniidae, les Trionychidae et les Testudininae: le contexte continental et la présence du spécimen précédent favorisent l'appartenance à un Testudininé. Il est rapporté au taxon du spécimen précédent provisoirement, en attendant que sa structure d'oeuf de tortue terrestre soit vérifiée.

\section{COMPARAISON}

Les caractères du spécimen de carapace de Lissasfa sont très proches de ceux de C. sulcata; il est rapporté à Centrochelys mais il représentait une espèce miocène sûrement distincte. Les proportions sont un peu différentes mais restent du domaine de la différence spécifique: la saillie gulaire est faible (un peu érodée), à la différence de $C$. sulcata actuelle (Lapparent de Broin 2003: pl. 3, fig. 1), d'un spécimen holocène du Niger (Roset et al. 1990) et du spécimen de $C$. aff. sulcata du Miocène supérieur d'Abu Dhabi (Lapparent de Broin \& Van Dijk 1999); les deux derniers ont une saillie un peu plus forte et ils sont tous deux des femelles, la saillie y étant cependant bien moins forte que chez les mâles actuels et non fourchue comme elle l'est chez ceux-ci (Vaillant 1885). Les gulaires atteignent l'entoplastron comme sur le spécimen d'Abu Dhabi et à la différence de l'actuel et de celui de l'holocène. L'entoplastron est grand également mais moins large et plus arrondi postérieurement, étant plus large que long chez l'actuel et probablement chez le spécimen holocène d'après le bord de l'épiplastron (non connu à Abu Dhabi). Les pectorales sont un peu plus longues, étant plus proches de l'entoplastron que chez l'actuel. Les bords latéraux des xiphiplastrons sont convergents vers l'arrière sous les anales au lieu d'être parallèles (après le même resserrement fémoro-anal) chez C. sulcata actuel (Lapparent de Broin 2003: pl. 3, fig. 1). La morphologie commune à l'espèce du Maroc et à l'actuelle Centrochelys sulcata s'oppose majoritairement à celle de l'autre moyen à grand genre d'Afrique Stigmochelys, réparti tout au long de la partie orientale jusqu'au Cap, du Miocène supérieur du lac Baringo (récolte M. Pickford) à l'époque actuelle (Lapparent de Broin 2000a) généralement avec des tailles modérées: chez Stigmochelys, le plastron est plus étroit, correspondant à une carapace plus bombée et plus étroite, les sillons huméro-pectoraux, pectoro-abdominaux et abdomino-fémoraux sont plus courbés, l'encoche anale est plus grande et le resserrement fémoroanal est moins fort (Meylan \& Auffenberg 1987; Harrison 2011; Lapparent de Broin 2003: pl. 3 , fig. 2). Les différences capitales entre Centrochelys et Stigmochelys sont la forme de la carapace, basse chez le premier et haute et plus étroite chez le second, et la forme du bourrelet épiplastral dorsal qui est recourbé et convexe chez le second au lieu d'aplati et concave chez Centrochelys: c'est pourquoi il est dommage de ne pas disposer de la morphologie du bourrelet chez le spécimen du Maroc; mais la forme basse et les autres caractères permettent sans peine de l'attribuer à Centrochelys, sans toutefois pouvoir nommer l'espèce. Une autre espèce géante existait au Miocène d'Afrique en Namibie, Namibchersus namaquensis (Stromer, 1926) mais la morphologie du plastron est différente: plastron étroit à grand lobe antérieur anguleux, lobe postérieur non resserré aux anales etc. (Lapparent de Broin 2003, 2008); c'est une forme dont la carapace, haute, a gardé l'écaille cervicale et elle est plus proche des petits endémiques africains.

Le petit plastron de Lissasfa est distinct de celui de la grande "Ch.» perpiniana dont le lobe antérieur est plus trapézoïdal et allongé médialement avec de ce fait une plus grande distance entre les gulaires et l'entoplastron et un bourrelet dorsal épiplastral très long et convexe-aplati; le lobe postérieur à des bords moins convergents vers l'arrière, aussi resserrés au sillon fémoro-anal mais avec une encoche anale plus large (Depéret \& Donnezan 1890-1897); les autres caractéristiques (écailles à sillons rectilignes et leur position relative, entoplastron grand) sont 
assez semblables à celles du spécimen de Lissasfa et de $C$. sulcata, d'où la confusion entre les grands "Cheirogaster» néogènes européen et Centrochelys (voir Čhkhikvadze 2000; Danilov 2005).

Ainsi, par ailleurs, la morphologie du plastron de Lissasfa est proche de celle d'un taxon du Miocène inférieur, Burdigalien (Orléanien, MN 4) (Tableau 1) d'Artenay, France: d'abord nommé Testudo ginsburgi Broin, 1977, sur la base d'un petit plastron assez jeune (MNHN.F.ART541; Broin 1977: pl. 32, fig. 1; pl. 33, fig. 1) (long de 236 sur $120 \mathrm{~mm}$ de large au sillon abdomino-fémoral), il s'est avéré qu'il n'appartenait ni à Testudo ni à Paleotestudo quand ces genres furent distingués et il fut rebaptisé Cheirogaster ginsburgi par Lapparent de Broin (2000b). Le plastron "Ch.» ginsburgi d'Artenay ressemble à celui de Lissasfa par sa face ventrale. Il se distingue de l'actuel C. sulcata, comme le spécimen de Lissasfa: 1) par les bords latéraux des xiphiplastrons un peu obliques et convergents vers l'arrière sous les anales au lieu de parallèles (après le même fort resserrement fémoro-anal), quoique les xiphiplastrons soient un peu moins obliques sous les anales que sur le plastron de Lissasfa; 2) par la longueur des gulaires jusque sur l'entoplastron; et 3) et par la moindre saillie gulaire, même pour une femelle ou un jeune adulte. Les sillons sont aussi rectilignes que chez $C$. sulcata mais, chez "Ch." ginsburgi et à Lissasfa, les humérales sont plus courtes par le sillon huméro-pectoral contactant l'entoplastron chez "Ch. " ginsburgi et très proche de lui chez Lissasfa; et enfin, l'entoplastron est arrondi chez « Ch. " ginsburgi comme à Lissasfa au lieu de plus large que long chez C. sulcata. Mais l'espèce ginsburgi se distingue de tous les spécimens de Centrochelys actuel et fossiles de l'Holocène comme d'Abu Dhabi, par son bourrelet épiplastral, relativement court antérieurement, à bord postérieur plus arrondi, plus large, plus long jusqu'à l'entoplastron, et dorsalement très aplati sans être concave (Broin 1977: pl. 33, fig. 1). Le bourrelet dorsal épiplastral est saillant, élevé et en pente forte chez l'espèce type $C h$. maurini, il est trapézoïdal, allongé et convexe-aplati chez "Ch.» perpiniana, montrant la variabilité entre les formes européennes néogènes. "Ch." ginsbugi, "Ch." perpiniana (Depéret \& Donnezan 1890-1897: pls 14, 15) et l'espèce type Ch. maurini (Broin 1977: fig. 105 ; pl. 26, figs 9, 10) ont d'autres caractères les différenciant mais c'est la découverte de plusieurs fossiles d'âges différents à bourrelet de type C. sulcata en Afrique et Arabie qui a montré l'importance du caractère de la concavité ou convexité du bourrelet pour le genre (différence concavité-convexité à rapprocher de la différence entre le clade T. kenitrensis et les autres espèces de Testudo). Les "Cheirogaster" (néogènes) incluant le spécimen d'Artenay comme celui de Perpignan sont cependant morphologiquement proches de Centrochelys comme cela a été vu avec ?C. marocana n. sp. et une proche parenté entre eux reste possible. Ainsi, un fémur inédit de grande taille (276 mm de longueur) qui accompagne le petit plastron de "Ch." ginsburgi et peut être rapporté à l'espèce, est très proche de celui de "Ch." perpiniana: à la différence de Testudo ou de Stigmochelys (à carapace bombée), ce fémur a une diaphyse presque droite mais tout de même un peu courbée vers la partie distale avant la facette articulaire, comme à Perpignan. Il se distingue du fémur de C. sulcata, de celui de ?C. punica et du fémur du Miocène inférieur du Djebel Zelten dont la diaphyse est rectiligne jusqu'à la base distale. Il semble donc bien possible de distinguer d'une part un ou des genres européens (pour les espèces « $C h$.» ginsburgi, "Ch." perpiniana et "cf. Ch." schafferi) et d'autre part le genre africain Centrochelys, par la morphologie du bourrelet épiplastral comme par la diaphyse du fémur et des humérus tout en leur reconnaissant une possibilité de parenté proche.

\section{DISCUSSION}

D'Europe occidentale jusqu'au Proche-Orient, les Testudininae géants du Néogène ont été attribués à "Cheirogaster» (sans écaille cervicale) et "Ergilemys» (avec encore une écaille cervicale) (Broin 1977; Lapparent de Broin 2000b, 2001, 2002). Mais l'on sait qu'il convient de redéfinir ces genres et de nommer la forme de l'Éocène inférieur. En Afrique, Raynal et al. (1990) avaient déjà signalé à Ahl al Oughlam la présence d'une tortue terrestre, de forme géante comme la «Testudo punica» Aramboug, 1979, du Lac Ichkeul. Ce matériel a été attribué par ces auteurs, puis par Bailón (2000), à Geochelone sp.; Lapparent de Broin (2000a) l'attribue à ?Centrochelys et il 
est nommé ici ?C. marocana n. sp. Entre temps, il a été démontré que "Geochelone» est un genre compréhensif artificiel, paraphylétique, dont le nom est généralement donné abusivement aux grandes tortues terrestres du monde entier (Lapparent de Broin 2000a, 2002, 2003). Au Maghreb, le problème était de savoir si les grandes formes d'Ahl al Oughlam retrouvées par des restes isolés appartenaient au genre Centrochelys ou à un autre genre. Les grands spécimens fossiles d'Afrique se répartissent en plusieurs genres (Lapparent de Broin 2000a, 2002, 2003, 2008) et parmi eux Centrochelys: la présence de l'espèce $C$. sulcata est prouvée à l'Holocène ancien d'Afrique du nord (Lapparent de Broin 2000a), par exemple au Niger (Roset et al. 1990: pl. 3, fig. 5) et elle est estimée dès le Pliocène, par exemple au Tchad à Ouadi Derdemi (Lapparent de Broin 2000a). Elle est prouvée au Miocène supérieur des Emirats Arabes Unis (Lapparent de Broin \& Van Dijk 1999). Centrochelys est cité avec Stigmochelys au Plio-Pléistocène d'Ethiopie (Omo, Hadar) par des formes géantes inédites observées par deux d'entre nous et conservées à Addis Abeba (Ethiopie) (Broin 1979); enfin, sa présence est estimée possible au Miocène inférieur (Burdigalien) de Djebel Zelten (Libye) et d'As Sarrar (Arabie Saoudite) (Lapparent de Broin 2000a). C'est de Centrochelys que sont alors rapprochées les formes d'Ahl Al Oughlam, de Djebel Krechem El Artsouma et d'Ichkeul. Si la ressemblance globale avec Centrochelys de la face ventrale des plastrons a amené à lui attribuer des "Cheirogaster" fossiles (Čhkhikvadze 2000 ; Danilov 2005), un examen plus complet permet de séparer les formes africaines et européennes et principalement à l'aide de la morphologie du bourrelet épiplastral dorsal. Malgré leur préservation incomplète les restes de ces grandes tortues terrestres du nord de l'Afrique sont certainement plus proches de Centrochelys que de Stigmochelys et potentiellement apparentés à un complexe "Cheirogaster» donné préalablement comme groupe-frère de Centrochelys (Lapparent de Broin 2000b, 2002). Des migrations de tortues ont eu lieu entre Europe et Afrique du nord à partir du Miocène tel qu'avec les représentants de la faune paléarctique terrestre et d'eau douce
(Testudo, Mauremys) attestés depuis la péninsule d'Arabie jusqu'au Maroc (Gmira 1995; Lapparent de Broin 2000a) et des relations ne sont pas interdites pour les formes géantes. Etant donné la relative faiblesse des restes fossiles africains géants, c'était avec plus de doute qu'étaient attribués les restes néogènes du Maghreb au taxon Centrochelys: la présente comparaison montre quelques ressemblances réelles entre les pièces géantes du Maghreb et Centrochelys et d'autre part la présence du genre Centrochelys au Maroc dès le Miocène supérieur/base du Pliocène semble attestée à Lissasfa. La préparation du spécimen de Lissasfa est nécessaire, mais en tout état de cause la présence de Centrochelys au Miocène supérieur et au Pliocène du Maroc est ici considérée comme vraisemblable. ?Centrochelys marocana $\mathrm{n}$. sp. est distincte des autres espèces rapportés à ce genre par les particularités des éléments préservés. En fin de compte son appartenance à Centrochelys, mériterait d'être confirmée mais elle est crédible.

\section{Sous-famille GeOEMYdinaE Theobald, 1868 Genre Mauremys Gray, 1869}

\section{Mauremys cf. leprosa (Schweigger, 1812)} (Fig. 11)

Matériel. - Fragment antérieur de nucale, AaO-680 (Fig. 11A); hyoplastron droit, AaO-671 (Fig. 11B).

LOCALITÉ ET HORIZON STRATIGRAPHIQUE. - Remplissage continental dans la carrière d'Ahl al Oughlam, située au sud-est de Casablanca, à gauche de la route S 106 allant de Casablanca à Tit Mellil. 3334'11'N, 07³0'44”W. Cordons dunaires de la côte de Casablanca à Kenitra, Maroc. Carte géologique, feuille de Casablanca-Mohammedia (Destombes 1987). Pliocène supérieur, âge estimé à 2,5 Ma (Raynal et al. 1990; Geraads et al. 2010).

\section{DESCRIPTION}

Nucale, AaO-680 (Fig. 11A)

Le fragment porte une écaille cervicale. Le bord préservé de la nucale de part et d'autre de la cervicale est légèrement sinueux. Celle-ci est plus longue que large dorsalement, saillant légèrement avec un contour arrondi à l'avant et un bord postérieur légèrement concave. Les écailles marginales 1 s'allongent 


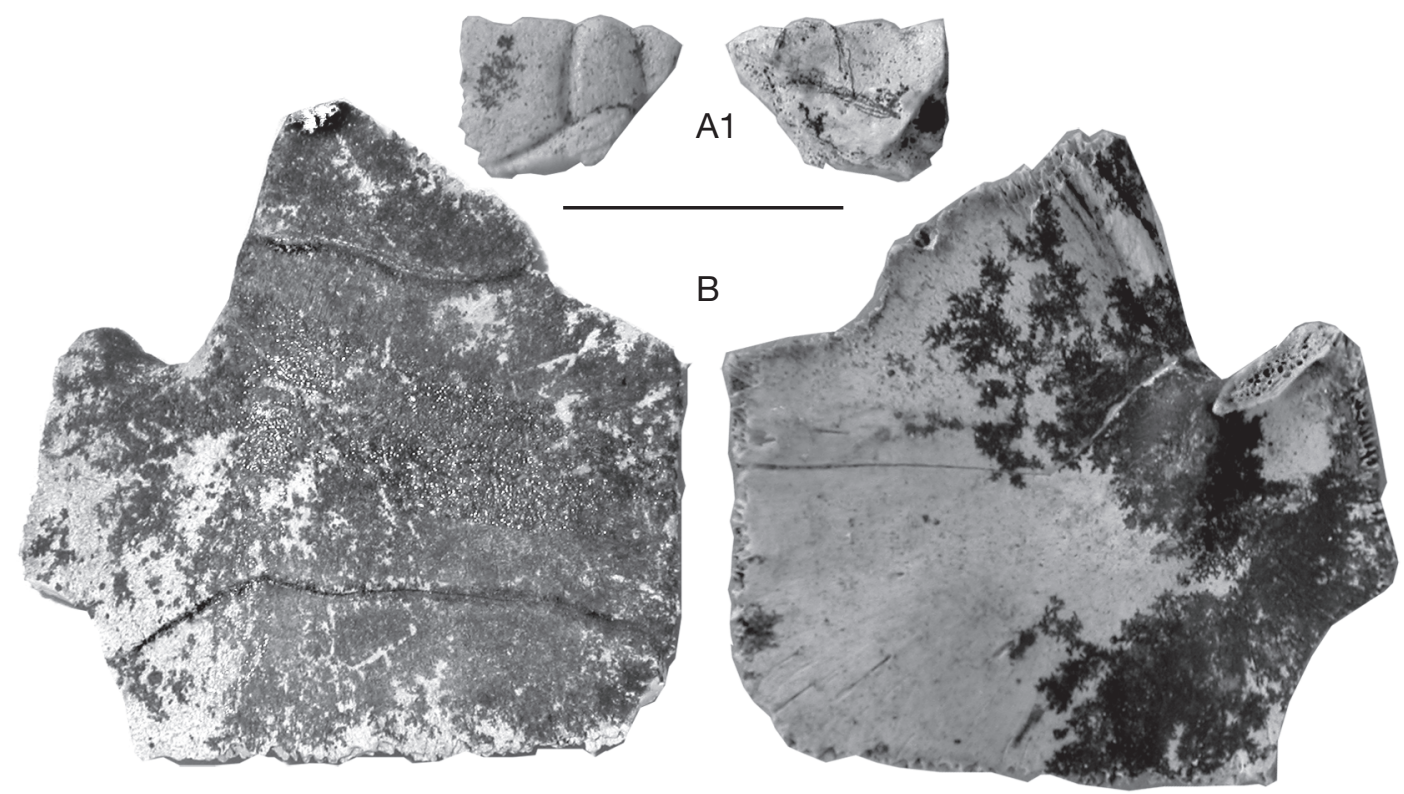

FIG. 11. - Mauremys sp., Pliocène supérieur, coll. INSAP. AaO-680 : A, fragment de nucale avec l'écaille cervicale, faces dorsale et ventrale, AaO-671; B, hyoplastron gauche, faces ventrale et dorsale. Échelle : $2 \mathrm{~cm}$.

postéro-latéralement à partir de la cervicale. Ventralement, le rebord des écailles cervicale et marginales 1 est relativement court; la cervicale ventrale est plus large que longue au milieu, un peu élargie à l'arrière, les écailles marginales 1 s'allongeant de chaque côté comme dorsalement en délimitant une douce concavité de la plaque, sans rebord fort.

\section{L'hyoplastron droit, AaO-671 (Fig. 11B)}

Aussi large que long au maximum, sa surface ventrale est plane et doucement courbée latéralement vers le processus hyoplastral; l'os est épaissi dorsalement vers le pont, mais il est mince globalement. Les bords latéraux du lobe antérieur sont convergents en léger arrondi, d'après cette seule partie hyoplastrale préservée et l'angle de l'encoche axillaire est de $c .60^{\circ}$. Les sillons sont sinueux. Le sillon huméro-pectoral est transversal et médialement il traversait l'entoplastron vers ses $2 / 3$ postérieurs. Par symétrie, l'entoplastron était pyriforme et large pour sa longueur hypothétique. Le sillon pectoro-abdominal traversait l'hyoplastron vers le quart arrière avec une sinuosité anguleuse à la courbure latérale du processus plastral.

\section{COMPARAISON}

Ces deux éléments sont conformes à ceux déjà décrits pour le Maroc, du Quaternaire, Soltanien terminal - à base de l'Holocène, de Doukkala 2 (Gmira 1995), et ils présentent les caractères de Mauremys leprosa (Schweigger, 1812), les différenciant de Testudo. La portion dorsale de la cervicale majoritairement plus longue que large chez l'adulte est un caractère dérivé qui différencie cette espèce de $M$. caspica (Gmelin, 1774) plus orientale et des formes ancestrales (Lapparent de Broin \& Van Dijk 1999; Hervet 2004) où la cervicale est plus large que longue. La cervicale n'a pas les bords rectilignes et l'étroitesse de Testudo oughlamensis n. sp. sur une nucale à bord saillant et le bourrelet de la cervicale et des marginales n'est pas saillant et long sous la nucale; l'hyoplastron a un angle plus ouvert à l'encoche axillaire; il est plus mince et plus plat ventralement et le sillon huméro-pectoral ne contourne pas l'entoplastron et le traverse, entoplastron qui est plus large pour sa longueur; et enfin il n'y a pas d'annuli de croissance. 


\section{DisCUSSION}

La découverte de l'espèce Mauremys cf. leprosa (ou très proche) à Ahl al Oughlam montre la plus grande ancienneté de la présence de Mauremys au Maroc où il existe toujours avec plusieurs variétés géographiques (Schleich 1996; Bour \& Maran 1998). L'échantillon est insuffisant pour distinguer cette population pliocène de celles actuelles. Mauremys est connu en Europe et particulièrement en France depuis l'Oligocène (Broin 1977; Hervet 2004). Il était encore présent au Pliocène de Perpignan et au Plio-Pléistocène ancien du Piémont (Italie) (Broin 1977; Hervet 2004). Il avait régressé en Europe occidentale ou disparu (Hervet 2000) au cours des glaciations quaternaires, tandis qu'il poursuivait sa différentiation en particulier dans le nord de l'Afrique (Lapparent de Broin 2000a). Dans le continent africain, la ou les formes présumées ancestrales des espèces actuelles $M$. caspica et $M$. leprosa sont connues dans la péninsule Arabique à Abu Dhabi depuis le Miocène supérieur (Lapparent de Broin \& Van Dijk 1999), en Égypte depuis le Pliocène basal à Wadi Natrun (Dacqué 1912) et en Tunisie au Pliocène d'Ichkeul (notamment MNHN.F, 1950-1-117). L'espèce M. leprosa (à cervicale majoritairement étroite), seule présente au Maghreb actuellement comme dans la péninsule Ibérique et le sud de la France (ponctuellement), pouvait être déjà distinguée de la forme ancestrale et de $M$. caspica (à cervicale toujours large) dans le Maghreb au Pléistocène inférieur de Aïn Boucherit (Algérie) vers $2 \mathrm{Ma}$ (coll. MNHN.F 1953-22-26 et 27) (Tableau 1). Mais à Aïn Boucherit, la cervicale y est aussi large que longue, ce qui ne permet pas d'attester la présence de l'espèce. Il en est de même au Pléistocène de Tighenif (encore connu comme Ternifine ou Palikao) (Algérie) (notamment MNHN.F 1955-13), c. 700000 ans BP. Mauremys est présent dans bien d'autres localités pléistocènes du Maghreb telle Mansourah (Algérie; voir Thomas 1878) (vers un Ma) (Geraads 2010b) avec le moulage interne d' "Emys» prosigriz Thomas, 1878 (MNHN.F 1874-47) (Lapparent de Broin 2000a); d'autres restes insuffisants, parfois présentés sous le nom d' "Emys» (par confusion), ne permettent pas d'aller à l'espèce mais seulement au genre Mauremys dans le meilleur des cas, quand les spécimens se trouvent au Muséum de Paris et ont pu être vérifiés. Au Maroc, la mention de $M$. leprosa au Pléistocène de Taforalt (MNHN.F, récolte Roche 1953) était considérée comme la plus ancienne (Lapparent de Broin 2000a) avant Doukkala (Gmira 1995); mais cette localité s'est révélée comme probablement plus récente que Doukkala 2 (Geraads 2010b) et l'ancienneté revient maintenant à $\mathrm{Ahl}$ al Oughlam (Tableau 1). Mauremys leprosa a pu se diversifier au Maghreb pour pénétrer à partir du Maroc ou de l'Algérie la péninsule Ibérique (Lapparent de Broin \& Antunes 2000) puis le sud de la France, de retour après les glaciations quaternaires, où elle est attestée au Chalcolithique (Hervet 2000) et où elle survit actuellement, tandis que $M$. caspica s'est répartie dans le bassin méditerranéen oriental.

\section{RÉSULTATS}

L'étude de la faune d'Ahl al Oughlam, enrichie de sa comparaison avec celle de localités voisines en provenance de cordons dunaires de la région de Casablanca, dont Ar Rehamna, Dehar Mouak et Lissasfa, a permis de mettre en évidence au MioPliocène une faune de tortues composée de trois genres, Testudo, Centrochelys et Mauremys. Cette faune est représentée par plusieurs espèces, même si l'échantillon ne permet pas encore de les nommer toutes. Elle est majoritairement terrestre en genres comme (surtout) en nombre de spécimens: Ahl Al Oughlam et les autres sites des cordons dunaires de Casablanca n'ont livré que des Testudinidae terrestres, à l'exception des deux rares restes de la tortue amphibie Mauremys. En accord avec le reste de la faune de reptiles (Bailón 2000) et en l'absence de grandes formes nageuses telle Trionyx triunguis (Forskål, 1775), cela indique un environnement sans grands fleuves et sans grandes étendues d'eau, sachant que cette espèce existait dans les grands lacs et fleuves au sud et à l'est du Maroc à partir de la limite Mio-Pliocène et jusqu'à l'Holocène (Broin 1983); elle y sera seulement ponctuellement présente près de la côte de Rabat à Temara au Pléistocène tardif (Gmira 1995; Lapparent de Broin 2000a).

La faune montre la forte diversification du genre paléarctique Testudo avec l'individualisation dès le 
Pliocène terminal du clade T. kenitrensis représenté à Ahl Al Oughlam par T. oughlamensis n. sp. Ce clade, individualisé indépendamment de $T$. graeca, pourrait faire partie d'un groupe kenitrensis initié dès le Pliocène inférieur avec l'apparition éventuelle de la lyre (Testudo sp. A de Dehar Mouak) puis de la dossière courte et arrondie sans long plateau, de la forte encoche antérieure (Testudo sp. B d'Ar Rehamna) et de la concavité du bourrelet épiplastral dorsal, laquelle caractérise le clade de T. oughlamensis n. sp.-T. kenitrensis (Testudo sp. B d'Ar Rehamna dérivant de ce clade ou le précédant). Cette progression permet tout au moins d'associer le spécimen d'Ar Rehamna au groupe kenitrensis plus large que le clade, mais encore informel en l'absence de spécimens suffisamment complets et nombreux. La permanence au Pliocène du Maroc du genre africain Centrochelys, attesté au Miocène terminal de Lissafa, est présumée avec l'espèce géante ?C. marocana n. sp. à Ahl Al Oughlam. L'espèce d'Ahl Al Oughlam est la dernière géante du Maghreb après celle d'Ichkeul en Tunisie. La faune d'Ahl Oughlam et de ses environs complète largement dans le temps les témoignages au Maroc de la présence de ces genres.

\section{CONCLUSION}

La présente étude de la faune d'Ahl Al Oughlam et des ses alentours permet de faire le point, après les études antérieures (Gmira 1993a, 1995), sur le peuplement du Maroc par les tortues cryptodires continentales Testudinidae venues d'Eurasie. L'Afrique voit arriver des tortues cryptodires continentales d'Eurasie en plusieurs vagues à partir de l'Éocène supérieur. Celles-ci s'épanouissent partout en Afrique et notamment dans le Nord de l'Afrique aux dépens des populations gondwaniennes de pleurodires d'eau douce, qui en disparaissent en se cantonnant plus au sud (Broin 1983; Roset et al. 1990; Iverson 1992 ; Lapparent de Broin 2000a).

Centrochelys est considéré comme apparenté au genre Cheirogaster, connu en Europe dès l'Éocène supérieur et sous sa forme évoluée la plus proche de Centrochelys, dénommée ici "Cheirogaster", dès la base du Miocène inférieur (Aquitanien), date proche de la première attestation probable de Centrochelys en Afrique (Burdigalien). Le genre africain Centrochelys, restreint de nos jours vers le sud au Sahel (Broin 1983; Roset et al. 1990; Iverson 1992), n'était pas attesté auparavant au Maroc. Il était soupçonné dès le Miocène inférieur de Libye et d'Arabie saoudite, attesté dès le Miocène supérieur des Emirats Arabes Unis et de nouveau soupçonné notamment au Miocène supérieur et au Pliocène de Tunisie et d'Ahl Al Oughlam (Arambourg 1979; Geraads 1989; Raynal et al. 1990; Lapparent de Broin \& Van Dijk 1999; Bailón 2000; Lapparent de Broin 2000a): l'aire de répartition de Centrochelys est amplifiée au Maroc avec sa découverte au Miocène supérieur de Lissasfa et renforcée avec la description du spécimen pliocène d'Ahl $\mathrm{Al} \mathrm{Ou-}$ ghlam. Au Miocène et au Pliocène, et comme pour "Cheirogaster" en Europe, des formes attribuées à Centrochelys sont géantes, ?C. marocana n. sp. en étant l'une des plus grandes, sinon la plus grande, connues en Afrique à ce jour.

L'introduction au Maghreb de Testudo et Mauremys, genres paléarctiques européens comme Trio$n y x$, a pu se faire au Miocène un peu plus tard, à partir de l'est du Bassin méditerranéen. Le genre Testudo est connu en Europe à partir du Miocène supérieur de Grèce mais il pourrait aussi être connu dès le Miocène supérieur de Tunisie avec "Testudo" semenensis. Son lieu précis de diversification par rapport à Paleotestudo et Chersine ("Eurotestudo» in Lapparent de Broin et al. 2006a-c) reste donc inconnu mais possible dans l'est du bassin méditerranéen européen où il est le plus abondant dès le Miocène supérieur. Testudo apparaît bien diversifié au Pliocène avec les espèces du Maroc décrites ici et au Pléistocène-Holocène (Gmira 1995), T. graeca y restant seule à partir du Pléistocène supérieur. Notre connaissance de la date de différentiation du clade kenitrensis par rapport à l'actuelle Testudo graeca, différentiation attestée auparavant au Pléistocène moyen (Gmira 1995) et maintenant vers la fin du Pliocène, est reportée dans le temps ce qui laisse le temps à Testudo pour avoir été présent dans l'est du Maghreb au Miocène supérieur.

Il a été dit comment Mauremys, connu dès l'Oligocène en France, est connu dans le continent africain dès le Miocène supérieur des Emirats Arabes 
Unis et dès le Pliocène inférieur d'Egypte: l'espèce $M$. leprosa était présente au Pliocène supérieur du Maroc, différenciée par rapport à $M$. caspica présente actuellement de l'Arabie à l'est méditerranéen (Iverson 1992). Après la disparition des tortues pleurodires et la brève présence côtière de Trionyx au Pléistocène (Gmira 1995), M. leprosa est la plus ancienne tortue d'eau douce actuelle du Maroc. En effet Emys orbicularis (Linnaeus, 1758), laquelle n'est pas signalée fossile mais est bien répartie actuellement au Maroc, a dû arriver beaucoup plus récemment (Lapparent de Broin 2000a).

\section{Remerciements}

Nous remercions, pour leur assistance au MNHN, S. Bailón (ZA), L. Pierre et R. Bour (RA), C. Sagne et R. Allain (F), au MHNT, F. Duranthon, et pour leur appréciable contribution les rapporteurs A. Ohler et R. Bour (MNHN) et J. Claude (Montpellier). Le matériel de tortues du Maroc a été récolté dans le cadre du Programme "Casablanca» de l'Institut national des Sciences de l'Archéologie et du Patrimoine, co-dirigé par A. Mohib et J.-P. Raynal. Les fouilles sont financées par le Ministère des Affaires culturelles du Royaume du Maroc, le Ministère des Affaires étrangères et européennes, la Région Aquitaine, et le Department of Human Evolution du Max Planck Institute, Leipzig.

\section{RÉFÉRENCES}

ARAMBOURG C. 1952. — La paléontologie des vertébrés en Afrique du Nord française, 19 e Congrès géologique international, Alger 1952, Monographies régionales Hors série, $62 \mathrm{p}$.

ARAmbOURG C. 1979. - Vertébrés villafranchiens d'Afrique du Nord (Artiodactyles, Carnivores, Primates, Reptiles, Oiseaux). Éditions de la Fondation Singer-Polignac, Paris, $141 \mathrm{p}$.

Arambourg C. \& Piveteau J. 1929. - Les vertébrés du Pontien de Salonique. Annales de Paléontologie 18: 57-139.

BACHMAYER F. 1967. — Eine Riesenschildkröte aus den altpliozänen Schichten von Pikermi (Griechenland). Practica Akademiae Athenae 42: 303-317.

BAILÓN S. 2000. - Amphibiens et reptiles du Pliocène terminal d'Ahl al Oughlam (Casablanca, Maroc). Geodiversitas 22 (4): 539-558.
Bergounioux F. M. 1935. - Contribution à l'étude paléontologique des Chéloniens. Chéloniens fossiles du Bassin d'Aquitaine. Mémoires de la Société géologique de France N.S. 25: 1-216.

BergOunioux F. M. 1955. - Testudo semenensis nov. sp. du Djebel Demène (Tunisie). Bulletin de la Société des Sciences Naturelles de Tunisie 8 (1-2): 145-151.

BiberSON P. 1961. — Le cadre paléogéographique de la Préhistoire du Maroc Atlantique. Publication du Service des Antiquités du Maroc, Rabat 16, 235 (17): 544 p.

BouR R. 1994. - Recherches sur des Animaux doublement disparus: les Tortues géantes subfossiles de Madagascar. Mémoires et travaux de l'Institut de Montpellier de l'École Pratique des Hautes Études, Sciences de la vie et de la terre 19: 1-253.

Bour R. \& MARAn J. 1998. - Taxinomie de Mauremys leprosa (Schweigger, 1812) dans le sud du Maroc: la "Tortue aux yeux bleus» (Reptilia, Chelonii, Geoemydidae). Manouria 1 (2): 22-52.

Bour R. \& OHLer A. M. 2008. - Chersine Merrem, 1820 and Chersina Gray, 1831: a nomenclatural survey. Zootaxa 1752: 66-68.

Bourgat R. \& Bour R. 1983. — La Tortue géante de Perpignan. Société agricole, scientifique et littéraire des Pyrénées-Orientales 21: 167-177.

BROIN F. DE 1977. — Contribution à l'étude des Chéloniens. Chéloniens continentaux du Crétacé et du Tertiaire de France. Mémoires du Muséum national d'Histoire naturelle, Paris, C, 38: i-ix, 1-366.

BROIN F. DE 1978. — La poche à phosphate de Ste Néboule (Lot) et sa faune de vertébrés du Ludien supérieur. 3- Chéloniens. Paleovertebrata 8 (2-4): 181-190.

BROIN F. DE 1979. - Chéloniens du Miocène et du Pio-Pléistocène d'Afrique orientale. Bulletin de la Société géologique de France sér. 7, 21 (3): 323-327.

BROIN F. DE 1983. - Chéloniens 6, in BlanC-Vernet L., Broin F. de, Buffetaut E., Camps-Fabrer H., Carbonel P., Celles J.-C., Commelin D., Coude G., Dutour O., Fabre J., Faure M., Gast M., Gayet M., Guerin C., Hillaire-Marcel C., Kelner-Pillault S., Manière R., Petit-Maire N., Raimbault M., Riser J., Rognon P., Rosso J.-C., Schulz E., SERVANT-Vildary S. \& Soulié-Marsche I. (éds), Sahara ou Sahel? Quaternaire récent du Bassin de Taoudenni (Mali): 211-233.

ČKHIKVADZE V. M. 2000. - [The giant terrestrial turtles from the Western Palearctic of Late Tertiary], in Zhordania R. G. (ed), Actual Problems of Modern Biology, Academy of Tiflis: 20-35 [en russe, résumé en anglais].

Claude J. \& Tong H. 2004. - Early Eocene testudinoid turtles from Saint-Papoul, France with comments on the early evolution of modern Testudinoidea. Oryctos 5: 3-45.

COPPENS Y. 1971. — Les Vertébrés villafranchiens de Tunisie: gisements nouveaux, signification. Comptes 
Rendus de l'Académie des Sciences, Paris, 273, D: 51-54.

DACQUÉ E.1912. — Die fossilen Schildkröten Aegyptens. Geologische und Palaeontologische Abhandlungen 14 (N.S. 10) (4): 275-337.

DANILOV I. G. 2005. — Schildkröten (Testudines) II , 9. Die fossile Schildkröten Europas, in BÖHME W. (éd.), Handbuch der Reptilien und Amphibien Europas, Aula Verlag, Wiebeisheim, 3/IIIB: 327-441.

DePÉRET C. 1885. - Description géologique du Bassin tertiaire du Roussillon et description des vertébrés fossiles du terrain pliocène du Roussillon. Masson, Paris, $274 \mathrm{p}$.

Depéret C. 1890. - Sur la découverte d'une Tortue de terre géante au mont Léberon. Comptes rendus des séances de l'Académie des Sciences, Paris, 60, 17: 915-917.

Depéret Ch. \& Donnezan A. 1890-1897. - Classe des Reptiles, O. des Chéloniens: 140-168, in DepéRET C. (éd.), Les Animaux pliocènes du Roussillon. Mémoire de la Société géologique de France, Paléontologie 3: 1-194.

Destombes A. 1987. - Carte géologique du Maroc au 1/100 000e, - feuille N-I-29-XI-3 4, CasablancaMohammedia. Service Géologique du Royaume du Maroc, Ministère de l'Energie et des Mines, Direction de la Géologie, Notes et Mémoires 350.

EISENMANN V. \& GERAADS D. 2007. - The hipparion from the Late Pliocene of Ahl al Oughlam, Morocco, and a revision of the relationships of Pliocene and Pleistocene African hipparions. Paleontologia Africana 42: 51-98.

FalCONER H. \& CAUTley P. T. 1837. — On additional fossil species of the order Quadrumana from the Sivalik Hills. Journal of the Asiatic Society of Bengal 6: 358-360.

Fritz U. \& Kraus O. 2008. - Comments on "Chersine Merrem, 1820 and Chersina Gray, 1831: a nomenclatural survey by Bour \& Ohler, Zootaxa 1752: 66-68. Zootaxa 1893: 65-68.

Garcia G., Lapparent de Broin F. DE, Bonis L. De, Koufos G. D., Valentin X., Kostopoulos D. \& MerCeron G. 2011. — A new terrestrial Testudinidae from the Late Miocene hominoid locality "Ravin de la pluie" (Axios valley, Macedonia, Greece). 9th European Association of Vertebrate Palaeontologists Annual Meeting, 14-19 June 2011, Heraklion Crete, Greece, Poster.

GeraADS D. 1989. - Vertébrés fossiles du Miocène supérieur de Djebel Krechem El Artsouma (Tunisie Centrale). Comparaisons biodtratigraphiques Geobios 22: 777-801.

GERAADS D. 1995. - Rongeurs et Insectivores du Pliocène final d'Ahl al Oughlam, Casablanca, Maroc Geobios 28 (1): 99-115

GERAADS D. 1998. - Rongeurs du Mio-Pliocène de Lissasfa (Casablanca, Maroc) Geobios 31(2): 229-245.

GERAADS D. 2006. - The Late Pliocene locality of Ahl al Oughlam, Morocco: vertebrate fauna and interpretation. Transactions of the Royal Society of South
Africa 61 (2): 97-101.

GERAADS D. 2010a. - Biochronologie mammalienne du Quaternaire du Maroc atlantique, dans son cadre régional. L'Anthropologie 114 (3): 324-340.

GERAADS D. 2010b. - Biogeographic relationships of Pliocene and Pleistocene North-western African mammals. Quaternary International 212: 159-168.

GeraAds D. 2010c. - The Upper Pliocene Avifauna of Ahl al Oughlam, Morocco. Systematics and Biogeography. Records of the Australian Museum 62: 157-184.

GeraADS D. 2011. - A Revision of the fossil Canidae (Mammalia) of North-Western Africa. Palaeontology 54 (2): 429-446.

GeraAds D., Raynal J.-P. \& Sbihi-Alaoui F.-Z. 2010. Mammalian faunas from the Pliocene and Pleistocene of Casablanca (Morocco). Historical Biology 22 (13): $275-285$.

Gmira S. 1992. - Étude des Chéloniens fossiles du Maroc. Mémoire de thèse doctorale, Université Paris VII: $1-248$.

GMIRA S. 1993a. - Une nouvelle espèce de tortue Testudininei (Testudo kenitrensis n. sp.) de l'Inter Amirien-Tensiftien de Kénitra (Maroc). Comptes rendus de l'Académie des Sciences, Paris 316 (II): 701-707.

GMira S. 1993b. - Nouvelles données sur les espèces actuelles de Testudo (Chelonii, Testudinidae). Bulletin de la Société herpétologique de France 65: 49-56.

Gmira S. 1995. — Étude des Chéloniens fossiles du Maroc. Anatomie. Systématique. Phylogénie. Cahiers de Paléontologie, CNRS (ed), Paris, 140 p.

GraY J. E. 1831a. - Synopsis of the Species of the Class Reptilia: 1-110, in Griffith E. \& Pidgeon E. (éd.), The Class Reptilia arranged by the Baron Cuvier, with specific descriptions, in GrIFFITH E. (éd.), The Animal Kingdom arranged in conformity with its organization, by the Baron Cuvier ... with additional descriptions ..., vol. 9. Whittaker, Treacher and Co., London.

GraY J. E. 1831 b. - Synopsis Reptilium; or short descriptions of the species of Reptiles. Part I. Cataphracta. Tortoises, Crocodiles, and Enaliosaurians. Treuttel, Wurtz, and Co., London, 85 p.

Gunnell G. F., Thomas P., Eiting T. P. \& Geraads D. 2011. - New late Pliocene bats (Chiroptera) from Ahl al Oughlam, Morocco. Neus Jarbuch für Geologie und Paläontologie 260 (1): 55-71.

Harrison T. 2011. - Tortoises (Chelonii, Testudinidae), in HARRISON T. (ed.), Paleontology and geology of Laetoli: fossil hominins and the associated fauna. Vertebrate Paleobiology and Paleoanthropology 2 (17): 479-503.

HeRvet S. 2000. — Les Tortues du Quaternaire de France: critères de détermination, répartitions chronologique et géographique. Mésogée 58: 3-47.

Hervet S. 2004. - Systématique du groupe «Palaeochelys sensu lato - Mauremys» (Chelonii, Testudinoidea) du tertiaire d'Europe occidentale: principaux résultats. 
Annales de Paléontologie 90 (1): 13-78.

Highfield A. C. 1990. - Tortoises of North Africa: Taxonomy, nomenclature, phylogeny, and evolution with notes on field studies in Tunisia. Journal of Chelonian Herpetology 1 (2): 1-56.

HiraYAMA R. 1985. - Cladistic analysis of Batagurine turtles (Batagurinae: Emydidae: Testudinoidea). A preliminary result. Studia Geologica Salmanticensia, Vol. sp. 1. Studia Paleocheloniologica 1: 141-157.

IVERSON J. B. 1992. - A Revised Checklist with Distribution Maps of the Turtles of the World, Privately Print., Richmond, Indiana, 363 p., maps.

JAEGER J.-J. 1977. — Les rongeurs du Miocène moyen et supérieur du Maghreb. Palaeovertebrata 8 (1): 1-166.

LAPPARENT DE BROIN F. DE. 2000a. - African chelonians from the Jurassic to the Present. A preliminary catalog of the African fossil chelonians. Palaeontologia Africana 36: 43-82.

LAPPARENT DE BROIN F. DE. 2000b. - Les Chéloniens de Sansan, Mémoires du Muséum national d'Histoire naturelle, Paris 183 (11): 219-261.

LAPPARENT DE BRoIN F. DE 2001. - The European turthe fauna from the Triassic to the Present. Dumerilia 4, 3: 155-216.

LAPPARENT DE BROIN F. DE 2002. - A giant tortoise from the late Pliocene of Lesvos Island (Greece) and its possible relationships, in Proceedings of the 1st International Workshop On Late Plio-Pleistocene extinction and evolution in the Palearctic. The Vatera site, September 29-October 2, 1999, Polichnitos, Lesvos Island. Annales Géologiques des pays Hélléniques, Natural History Collection of Vrisa-Lesvos Island, 1re sér., 39, A: 99-130.

Lapparent de Broin F. DE 2003. - The Miocene chelonians from the southern Namibia, in SENUT B. \& PiCKFORD M. (éd.), Faunas from the southern Namibia. Memoirs of the Geological Survey of Namibia 19: 67-102.

LAPPARENT DE BROIN F. DE 2008. — Miocene Chelonians from Western Namibia. Memoirs of the Geological Survey of Namibia 20: 107-145.

LAPPARENT DE BRoIN F. DE \& ANTUNES M. T. 2000. Pleistocene Chelonians from Gruta da Figueira Brava (Arrábida, Portugal). Memorias da Academia das Ciências de Lisboa, Cl. Ciências 38:101-112.

Lapparent de Broin F. De, Bour R., Parham J. F. \& PERÄLÄ J. 2006a. - Eurotestudo, a new genus for the species Testudo hermanni Gmelin, 1789 (Chelonii, Testudinidae). Comptes Rendus Palevol 5: 803-811.

Lapparent de Broin F. de, Bour R. \& Perälä J. F. 2006b. - Morphological definition of Eurotestudo (Testudinidae, Chelonii): First part, Annales de Paléontologie 92 (3): 255-304.

Lapparent de Broin F. DE, Bour R. \& Perälä J. F. 2006c. - Morphological definition of Eurotestudo (Testudinidae, Chelonii): Second part Annales de
Paléontologie 92 (4): 325-357.

Lapparent de Broin F. De \& VAN DijK P. P. 1999. Chelonia from the Late Miocene Baynunah Formation, Emirate of Abu Dhabi, United Arab Emirates: Palaeogeographic Implications, in WHYBROW P. J. \& Hill A. (eds), Fossil Vertebrates of Arabia. Yale University Press, New Haven and London 13: 136-162. LeFÈVRE D. 2000. - Du continent à l'océan. Morphostratigraphie et paléogéographie du Quaternaire du Maroc atlantique. Le modèle casablancais, vol. 3. Université de Montpellier III, Mémoire HDR, IIème partie: 99-299.

LEFĖVRE D. \& RAYNAL J.-P. 2002. — Les formations plio-pléistocènes de Casablanca et la chronostratigraphie du Quaternaire marin du Maroc revisitées. Quaternaire 13 (1): 9-21.

MeYlan A. P. \& Auffenberg W. 1987. - The Chelonians of Laetolil Beds, in LEAKEY M. D. \& HARRIS J. M. (éds), Laetoli. A Pliocene site in Northern Tanzania, 3. Reptiles and Birds, Clarendon press, Oxford: 62-78.

Mourer-Chauviré C. \& GeraAdS D. 2008. - The Struthionidae and Pelagornithidae (Aves: Struthioniformes, Odontopterygiformes) from the Late Pliocene of Ahl al Oughlam, Morocco. Oryctos 7: 169-194.

Mourer-Chauvirê C. \& GeraAds D. 2010. - The Late Pliocene avifauna of Ahl al Oughlam, Morocco. Systematics and biogeography, in BOLES W. E. \& WorThY T. H. (éds), Proceedings of the VII International Meeting of the Society of Avian Paleontology and Evolution, Records of the Australian Museum 62 (1): 157-184.

PerÄLÄ J. 2002a. — The genus Testudo Linnaeus, 1758 sensu lato (Testudines: Testudinidae): Phylogeny, Taxonomy, Conservation. PhD thesis. University of Bristol, School of Biological Sciences: i-xiv +328 p.

PERÄLÄ J. 2002b. - The genus Testudo (Testudines: Testudinidae): Phylogenetic inferences. Chelonii 3: 32-39.

Peters K. R. 1869. - Zur Kenntniss der Wirbelthiere aus den Miocenschichten von Eibiswald in Steiermark. Denkschriften der Akademie der Wissenschaften in Wien, Mathematisch-Naturwissenschafiliche Klasse 29: 111-124.

PieH A. \& PeräLä J. 2004. — Variabilität der Maurischen Landschildkröten (Testudo graeca Linnaeus, 1758 - Komplex) im zentralen und norwestlichen Marokko mit Beschreibung zweier neuer Taxa (Testudines: Testudinidae). Herpetozoa 17 (1/2): 19-47.

Rage J.-CL. \& SeN S. 1976. - Les amphibiens et les reptiles du Pliocène supérieur de Çalta (Turquie). Géologie méditerranéenne 3 (2): 127-134.

Raynal J.-P., Lefèvre D., Geraads D. \& El Graoui M. 1999. - Contribution du site paléontologique de Lissasfa (Casablanca, Maroc) à une nouvelle interprétation du Mio-Pliocène de la Meseta. Comptes rendus de l'Académie des Sciences, Sciences de la Terre et des Planètes, Paris 329(8): 617-622.

Raynal J.-P., Texier J.-P., Geraads D. \& Sbihi-Alaoui 
F.-Z. 1990. - Un nouveau gisement paléontologique du Plio-Pléistocène du Maroc: Ahl al Oughlam (ancienne carrière Deprez). Comptes rendus de l'Académie des Sciences, Paris, sér. II, 310: 315-320.

Roset J. P., Broin F. De, Faure M., Gayet M., GuÉRIN C. \& Mouchet F. 1990. — La faune de Tin Ouaffadene et d'Adrar Bous 10, deux gisements archéologiques de l'Holocène ancien au Niger nordoriental. Géodynamique 5 (1): 67-89.

SCHLEICH H. H. 1996. - Beitrag zur Systematik des Formenkreises von Mauremys leprosa (Schweigger), Marokko. Spixiana Suppl. 22: 29-59.
SZALAI T. 1931. — Schildkrötenstudien: I. Testudo Schafferi nov. sp., eine Riesenschildkröte aus dem Pliozän von Samos. Annalen des Naturhistorischen Museums in Wien 46: 153-157.

Thomas P. 1878. - Note sur une tortue fossile des terrains supérieurs du Mansourah (province de Constantine). Revue des Sciences naturelles de Montpellier: 22-27.

VAILLANT L. 1885. - Remarques complémentaires sur les tortues gigantesques de Madagascar. Comptes Rendus hebdomadaires des Séances de l'Académie des Sciences, Paris 100: 874-877.

Submitted on 26 February 2012; accepted on 11 September 2012; published on 27 September 2013. 